\title{
Dunior High
}

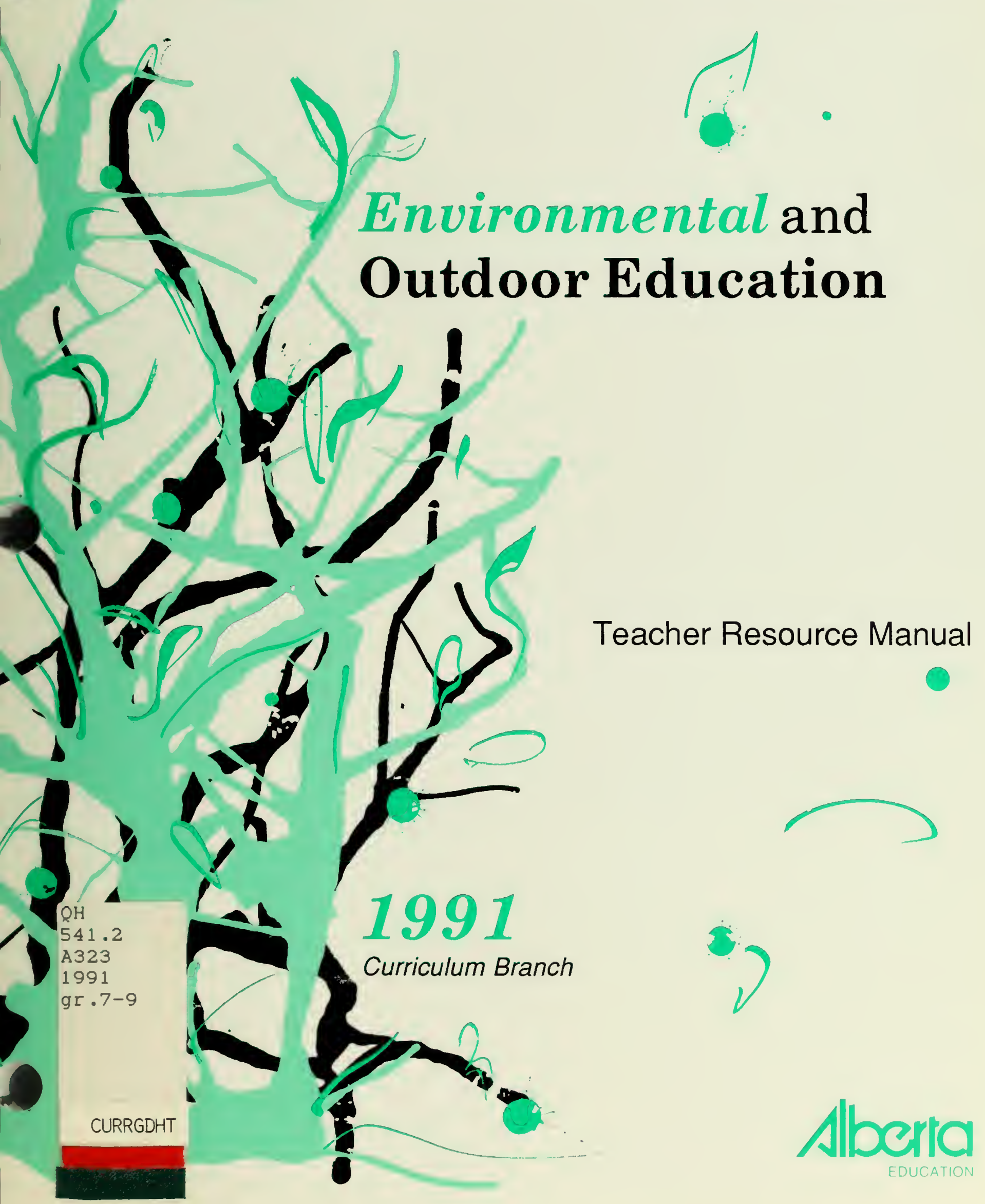




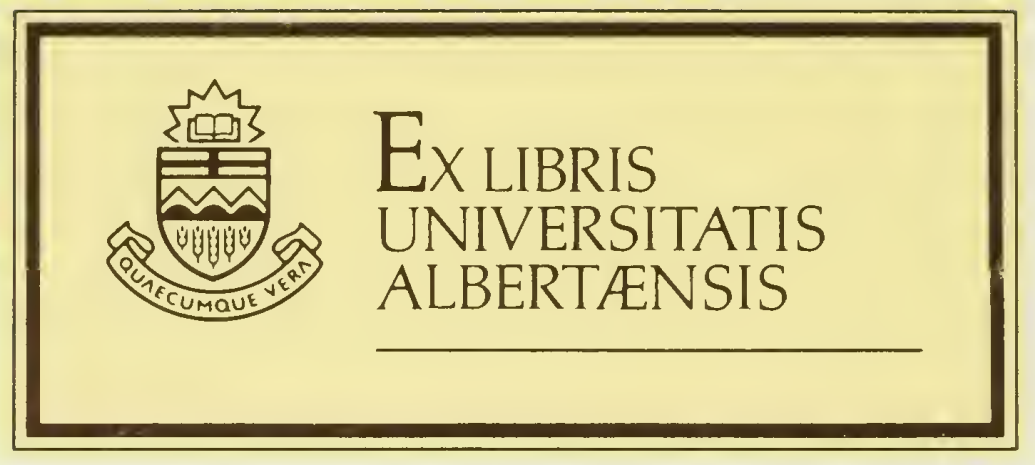




\section{JUNIOR HIGH}

\section{ENVIRONMENTAL \\ AND OUTDOOR EDUCATION}

\section{TEACHER RESOURCE MANUAL}

1991 
Alberta. Curriculum Branch.

Junior high environmental and outdoor education: teacher resource manual.

Cover title: Junior high school environmental and outdoor education teacher resource manual.

ISBN: $0-7732-0468-7$

1. Environmental education -Alberta. 2. Outdoor education - Alberta. I. Title.

LB1047.A333 1991 375.0083

Copyright 01991 , the Crown in Right of Alberta, as represented by the Minister of Education. Alberta Education, 11160 Jasper Avenue, Edmonton, Alberta, T5K OL2.

Permission is hereby given by the copyright owner for any person to reproduce this teacher resource manual or any part thereof for educational purposes and on a non-profit basis. Additional copies may be purchased from the Learning Resources Distributing Centre.

Additional copies may be obtained from the Learning Resources Distributing Centre at a nominal cost.

\author{
Learning Resources Distributing Centre \\ $12360-142$ Street \\ EDMONTON, Alberta \\ T5L 4X9
}

Note: This pubtication is a support document. The advice and direction offered are suggestions except where the course of studies is cluplicated or paraphrased. In these instances, the fext is screened in the same distinctive manner as inis notice so that the reader may readify identify all prescriptive statements of segments of the document. 


\section{ACKNOWLEDGMENTS}

Alberta Education expresses its appreciation to those individuals and organizations who assisted in the development and production of this manual and acknowledges the contributions of the following:

\section{ENVIRONMENTAL AND OUTDOOR EDUCATION ADVISORY COMMITTEE}

Ross Ash

Tom Bateman

Jim Brackenbury

Dennis Gogal

Garth Hendren

Nestor Kelba

Michael Mappin

Jim Martin

Brian Ogston

Donna Smith

Danica Stokoe

Gina Vivone
Cardston Junior High School, Cardston School Division Number 2

Fish and Wildlife Division, Alberta Forestry, Lands and Wildlife

Alberta Education

Steele Heights Junior High School, Edmonton Public School District Number 7

Alberta Education

Calgary School District Number 19

Kananaskis Centre for Environmental Research, University of Calgary

Communications Division, Alberta Environment

Alberta Recreation and Parks

Strathearn Junior High School, Edmonton Public School District Number 7

Glendale Junior High School, Red Deer School District Number 104

Alberta Education

\section{CALGARY ENVIRONMENTAL AND OUTDOOR EDUCATION STEERING COMMITTEE}

Al Brawn

Phil Carlton

Tim Cartmell

Dave George

Cal Kullman

Jim Latimer

Bill March

Bruce McLeod

Ric Million

Terry Polton

Jeff Reading

George Tàven

lan Waugh

\section{WRITERS}

\section{John Dupuis}

Bernie Galbraith

Nestor Kelba

Ron Sweet
Outdoor Pursuits Department, University of Calgary

Rideau Park Junior High School, Calgary Board of Education

Clarence Sansom Junior High School, Calgary Board of Education

Alberta Fish and Wildlife Division of Alberta Forestry, Lands and Wildlife

Bishop Pinkham Junior High School, Calgary Board of Education

Viscount Bennett Teacher Resource Centre, Calgary Board of Education

Outdoor Pursuits Department, University of Calgary

Annie Gale Junior High School, Calgary Board of Education

Annie Gale Junior High School, Calgary Board of Education

Sedimentary and Marine Geoscience Branch of Energy, Mines and Resources Canada

Environmental and Outdoor Education, Calgary Board of Education

Environmental and Outdoor Education, Calgary Board of Education

Kananaskis Country, Alberta Recreation and Parks

Alberta Education also acknowledges the field test teachers for Calgary public schools and other individuals throughout the province who have contributed to the development of this curriculum. 
Development Co-ordinator

Pamela Shipstone Alberta Education

Editor

Barbara McCord

Alberta Education

Desktop Publisher

Tania Pander

Alberta Education

Artwork

Jaclyn Peebles 


\section{TABLE OF CONTENTS}

CHAPTER 1 - Introduction

Course Rationale and Philosophy $\ldots \ldots \ldots \ldots \ldots \ldots \ldots \ldots \ldots \ldots \ldots \ldots \ldots$

- Needs of the Adolescent . . . . . . . . . . . . . . . . . . . . . . . . . . 3

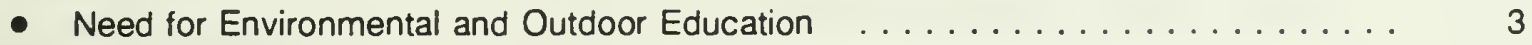

- Course Characteristics .............................. 4

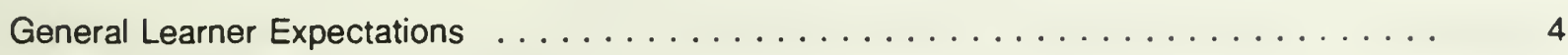

Course Organization . ............................ 4

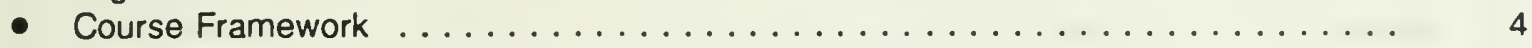

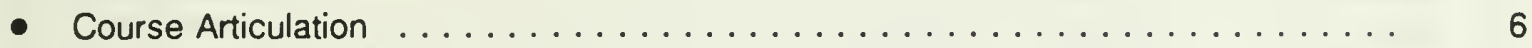

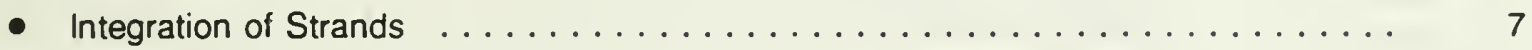

- Required/Elective Components ....................... 8

Planning Considerations $\ldots . \ldots \ldots \ldots \ldots \ldots \ldots \ldots \ldots \ldots \ldots \ldots$

- Environmental and Outdoor Education as Part of the School Program . . . . . . . 9

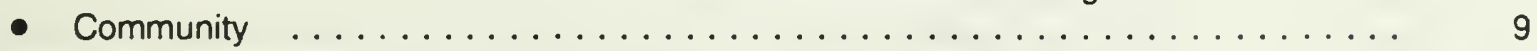

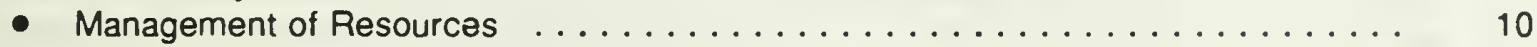

- Environmental impact .............................. 10

- Safety ................................... 10

- Balanced Perspective ............................ 10

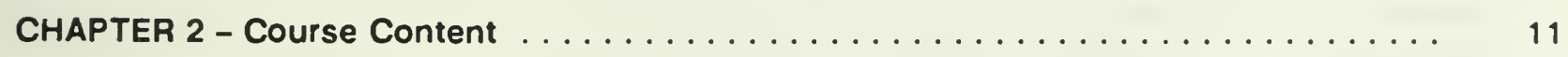

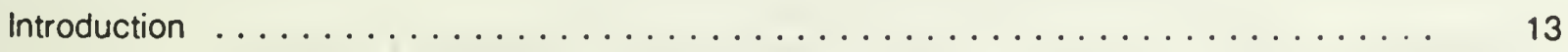

Content and Development Outlines $\ldots \ldots \ldots \ldots \ldots \ldots \ldots \ldots \ldots \ldots \ldots$

CHAPTER 3 - Resources $\ldots \ldots \ldots \ldots \ldots \ldots \ldots \ldots \ldots \ldots \ldots$

Authorized Resources $\ldots \ldots \ldots \ldots \ldots \ldots \ldots \ldots \ldots \ldots \ldots \ldots \ldots$

Other Resources $\ldots \ldots \ldots \ldots \ldots \ldots \ldots \ldots \ldots \ldots \ldots \ldots \ldots \ldots \ldots \ldots$

CHAPTER 4 - Unit Planning $\ldots \ldots \ldots \ldots \ldots \ldots \ldots \ldots \ldots \ldots \ldots$

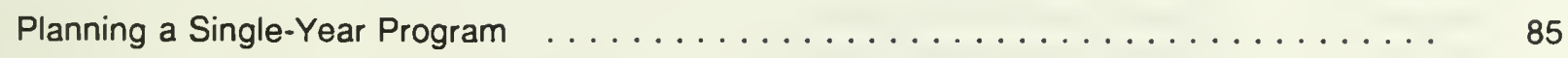

Planning a Multi-Year Program $\ldots \ldots \ldots \ldots \ldots \ldots \ldots \ldots \ldots \ldots$

Unit and Lesson Planning $\ldots \ldots \ldots \ldots \ldots \ldots \ldots \ldots \ldots \ldots \ldots \ldots$

- Year $1 /$ Unit 1 The Great Outdoors . . . . . . . . . . . . . . . . . . 87

- Year 1/Unit 2 We're Being Audited! ....................... 88

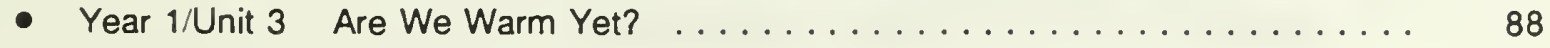

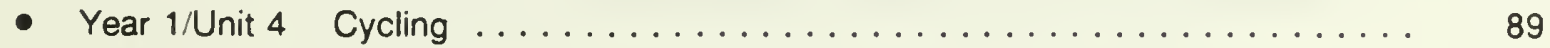

- Year 1 Unit 5 Spring Breakup ......................... 90 
- Year 2/Unit 1 Can't See The Forest For The Trees . . . . . . . . . . . . . . . 91

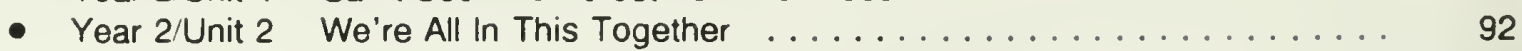

- Year 2 Unit 3 "Jack Rabbit" Johannsen . . Here We Come . . . . . . . . . . . . . 92

- Year $2 /$ Unit 4 Wide Open Spaces .......................... 93

- Year $2 /$ Unit 5 River Tripping .......................... 94

- Year 3: Starting Points for a Plan $\ldots \ldots \ldots \ldots \ldots \ldots \ldots \ldots \ldots \ldots$

CHAPTER 5 - Lesson Planning: A Model Introductory Unit . . . . . . . . . . . . . 97

Overview, Unit 1.1 The Great Outdoors $\ldots \ldots \ldots \ldots \ldots \ldots \ldots \ldots \ldots$

Lesson $1 \quad$ Journal Writing $\ldots \ldots \ldots \ldots \ldots \ldots \ldots \ldots \ldots \ldots \ldots \ldots$

Lesson $2 \quad$ Introduction and Planning ....................... 100

Lesson $3 \quad$ Getting to Know One Another . . . . . . . . . . . . . . . . . . 100

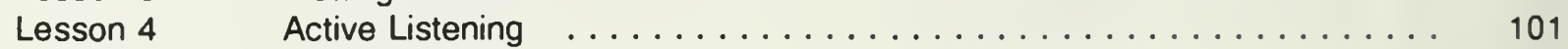

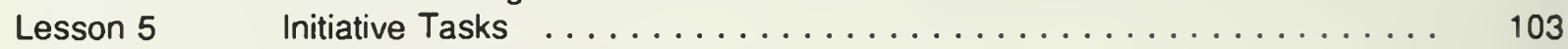

Lesson $6 \quad$ Outdoor Clothing ............................. 104

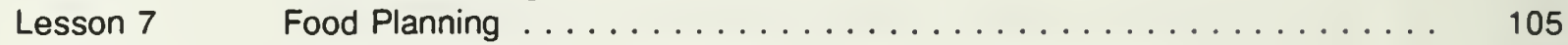

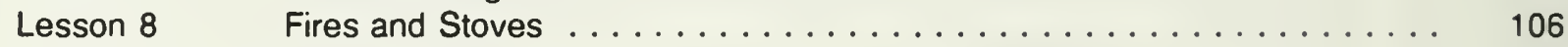

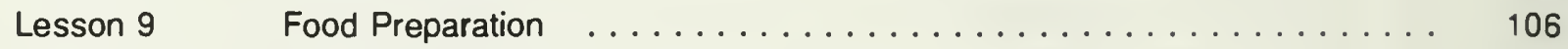

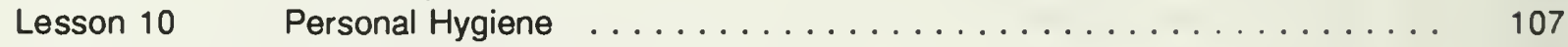

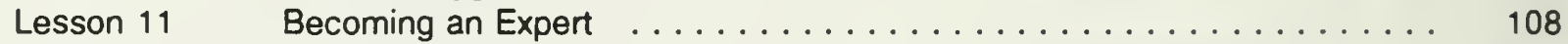

Lesson $12 \quad$ Interrelationships ............................ 109

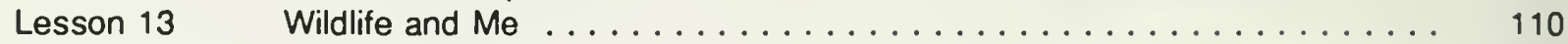

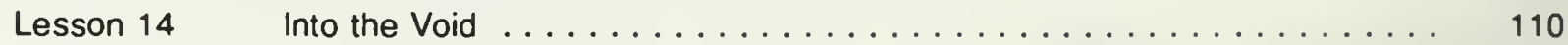

Lesson $15 \quad$ Let the Bells Ring Out $\ldots \ldots \ldots \ldots \ldots \ldots \ldots \ldots \ldots \ldots \ldots \ldots$

CHAPTER 6 - Lesson Planning: A Model Intermediate to Advanced Level Unit . . . . 113

Overview, Unit 2.5 River Tripping $\ldots \ldots \ldots \ldots \ldots \ldots \ldots \ldots \ldots \ldots \ldots$

Lesson $1 \quad$ Introduction of Unit, Learning Opportunities and Responsibilities . . . . . 115

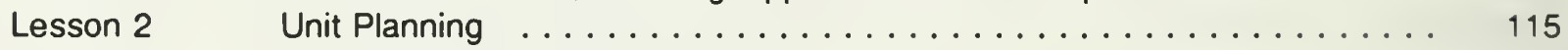

Lesson $3 \quad$ Review of Lake Canoeing Safety $\ldots \ldots \ldots \ldots \ldots \ldots \ldots$

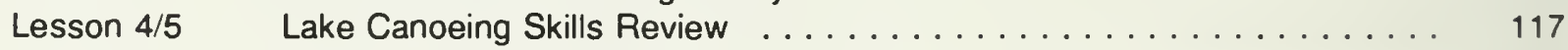

Lesson $6 \quad$ River Awareness (Session 1) . . . . . . . . . . . . . . . 118

Lesson $7 \quad$ River Awareness (Session 2) . . . . . . . . . . . . . . . . 118

Lesson $8 / 9 / 10 \quad$ Introduction to River Canoeing . . . . . . . . . . . . . . . . . . . 119

Lesson $11 \quad$ Map Skills ................................. 120

Lesson $12 \quad$ Wildlife Awareness . . . . . . . . . . . . . . . . . . . . . . 121

Lesson $13 \quad$ Clean Water and Its Value . . . . . . . . . . . . . . . . . . . . . . . 122

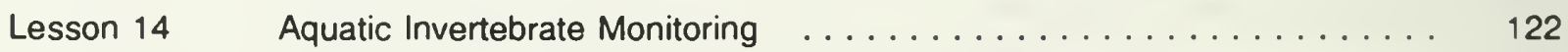

Lesson $15 \quad$ Aquatic Invertebrate Monitoring: Interpreting the Results . . . . . . . 123

Lesson $16 \quad$ Aquatic Invertebrate Monitoring: Verifying the Results . . . . . . . . . 124

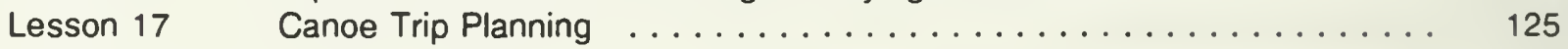

Lesson $18 \quad$ Canoe Tripping . . . . . . . . . . . . . . . . . . . . . . 126

Lesson $19 / 20$

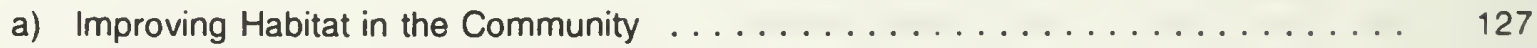

b) Monitoring and Reducing Water Consumption $\ldots \ldots \ldots \ldots \ldots \ldots \ldots$ 


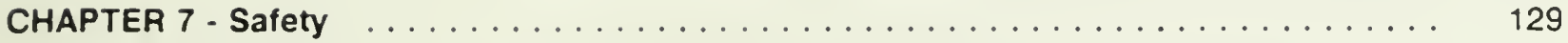

Planning and Conducting Safe Outdoor Trips . . . . . . . . . . . . . . . . 131

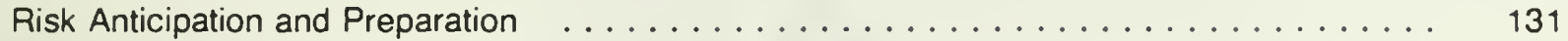

Ongoing Risk Assessment and Risk Reduction . . . . . . . . . . . . . . . . . 133

Emergency Response . . . . . . . . . . . . . . . . . . . . . . . . . . . . . . . . . . . . 134

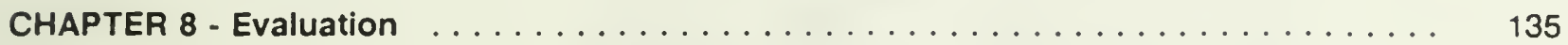

Evaluation Techniques ............................... 137

- Activity Reports and Projects . . . . . . . . . . . . . . . . . . . . 137

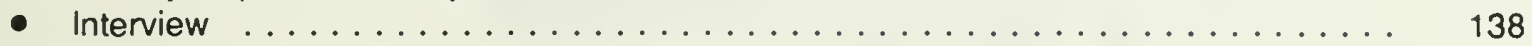

- Self-Evaluation ............................... 139

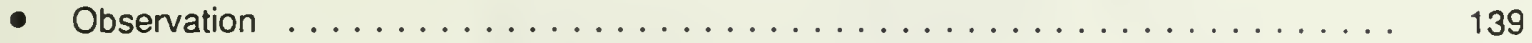

- Case Studies ................................... 139

- Expedition Evaluation . . . . . . . . . . . . . . . . . . . . . . . 140

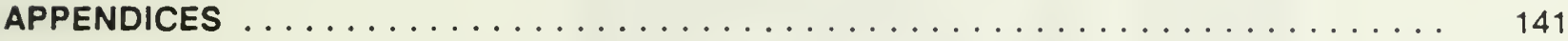

Appendix A: Alberta Education's Policy on Controversial Issues . . . . . . . . . . . . . . 143

Appendix B: Supplementary Information Sources . . . . . . . . . . . . . . . . . . 145 
Digitized by the Internet Archive in 2012 with funding from University of Alberta Libraries 


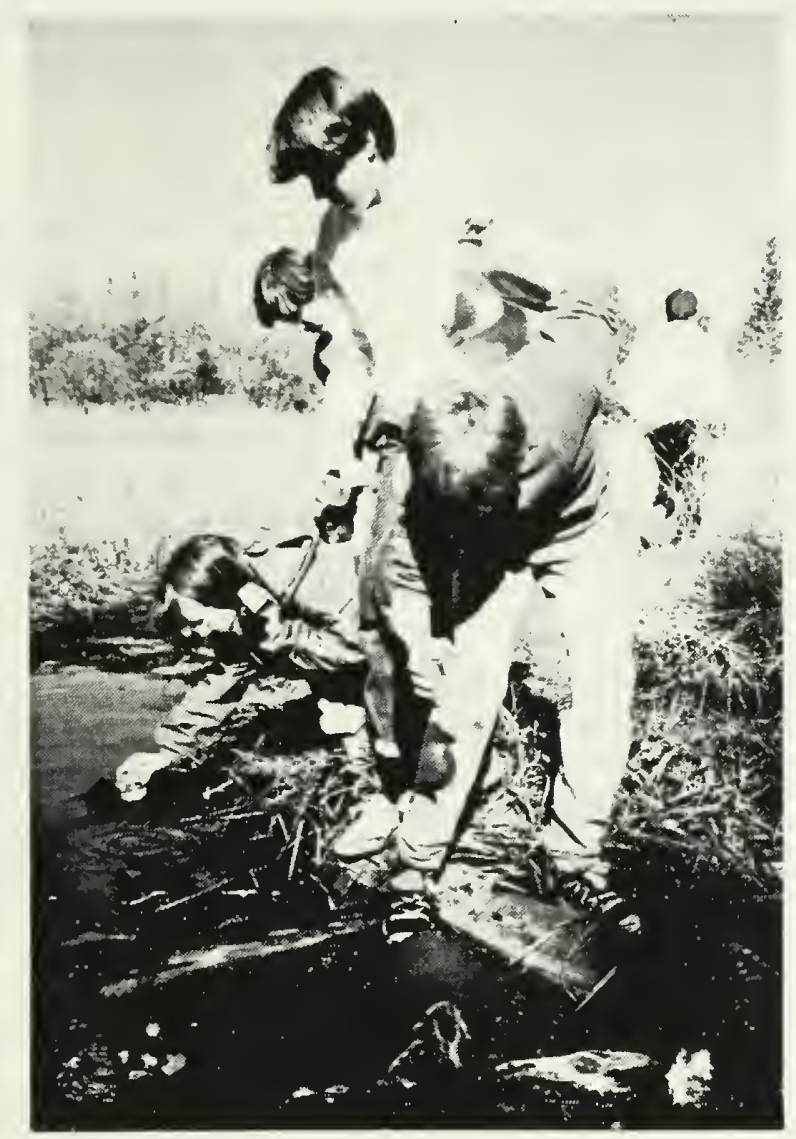

Chapter

\section{Introduction}

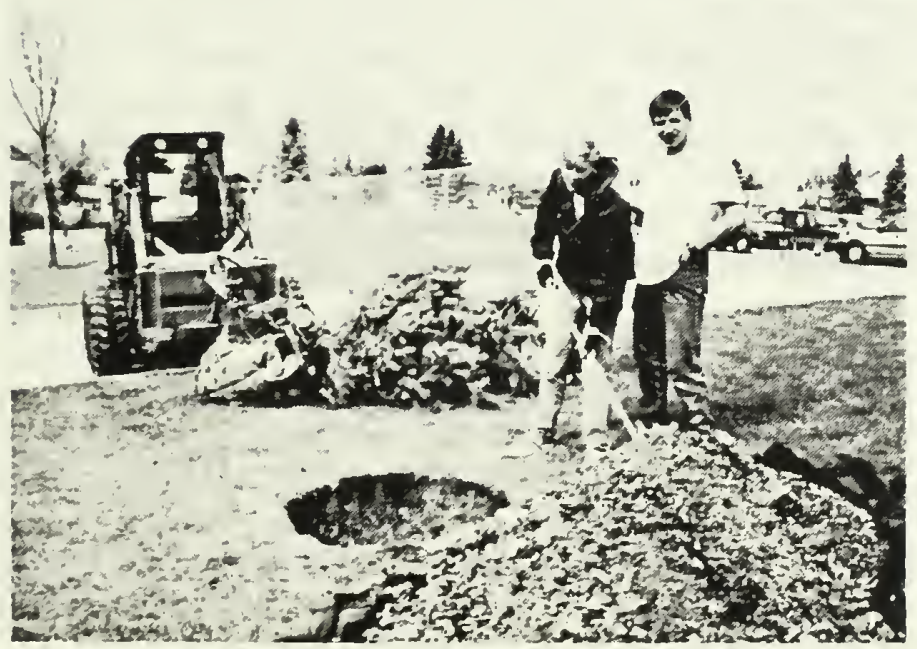





\section{COURSE RATIONALE AND PHILOSOPHY}

"The aim of education is to develop the knowledge, the skills and the positive attitudes of individuals, so that they will be self-confident, capable and committed to setting goals, making informed choices and acting in ways that will improve their own lives and the life of their community" (Secondary Education in Alberta 1985).

The Junior High Environmental and Outdoor Education Course supports the above program aim. The course design reflects an integrated approach that addresses the special needs of the adolescent student and can be offered to the student over one, two or three years.

\section{NEEDS OF THE ADOLESCENT}

The adolescent years are a time of learning and adjustment. A junior high student needs to make sense of the world in a personal way, establishing a sense of self through personal challenges, and establishing new relationships with peers, others and the environment. The junior high years are the time for students to lay solid foundations, explore new horizons and apply new-found knowledge, skills and values to the world around them.

Within their experience as adolescents, junior high students actively strive to develop a sense of identity by establishing and extending personal competencies. Establishment of physical skills that are clearly and immediately recognizable is of tremendous importance to students at this level. In many instances the motivation is not only the personal pride that comes with accomplishment, but also a feeling of social belonging that comes from taking part in enjoyable and meaningful activities with others. The quest for personal identity and wholeness is of utmost importance to the adolescent.

The holistic nature of the Junior High Environmental and Outdoor Education course provides an opportunity for lifelong learning while meeting the immediate needs of the adolescent.

\section{NEED FOR ENVIRONMENTAL AND OUTDOOR EDUCATION}

At the same time as the adolescent learner is trying to come to terms with the world, the world itself is changing. Our local and global environments are undergoing transformation at a rapid pace and at a scale that is unparalleled at any time in human history. It is now apparent that all environments are subject to the actions of humankind and collectively these actions strain the ability of the earth's systems to maintain themselves in balance.

Increasingly there is a need for our students to understand the consequences of human actions on environments. The effects of lifestyles and technology are in many cases not immediately apparent to students often because they are too difficult to predict, or are removed from them in time or space. In many instances students may not be aware of where the resources we use come from, or at what cost or with what long-term consequences. It is through the study of environments in a variety of urban, rural and natural settings that these effects become most evident. The student entering a relatively undisturbed area for study and enjoyment sees in a most tangible way the effects that previous users of the area have had. With some focus of attention it can become clear how group and personal actions have environmental consequences.

This personal awareness and knowledge can be extended through further study of environments, the interrelationships within them and ways in which we can manage local and global environments for the good of all living things. The Junior High Environmental and Outdoor Education course provides an opportunity for the integration of personal experience and formal learning. The course provides for growth of the student as an individual and as a responsible citizen. It will assist students in their quest to live harmoniously with others and with the earth. 


\section{COURSE CHARACTERISTICS}

This activity-based course will provide opportunities for in-depth practical studies and experiences. This direct approach should appeal to all students, particularly those who learn best from physical involvement. Students will learn by building personal skills and through assuming responsibility for individual and group welfare.

Decision-making skills will be emphasized. These will include personal, group and societal decisions and will involve students in the examination of personal and interpersonal consequences of their actions as well as the importance of their relationship with the environment.

\section{GENERAL LEARNER EXPECTATIONS}

Students will:

- demonstrate the basic knowledge, skills and attitudes necessary for safe, comfortable, outdoor experiences in all seasons

- demonstrate understanding, respect and appreciation for self, others and their views

- demonstrate an awareness and appreciation of living things and an understanding of basic ecological processes

- demonstrate skill, judgment, confidence and sensitivity through participation in a wide range of environmentally responsible activities in outdoor settings

- develop knowledge and skills by investigating the effects of human lifestyles on environments

- develop lifestyle strategies that foster contact with the natural world, demonstrate responsibility for local and global environments, and encourage living in harmony with others.

\section{COURSE ORGANIZATION}

\section{COURSE FRAMEWORK}

The Junior High Environmental and Outdoor Education (EOE) Course may be offered as a single course at the Grade 7,8 or 9 levels, or as a sequential program of up to three years. The course consists of six elements, each stemming from one of the six general learner expectations. The course elements are:

- Outdoor Core

- Personal and Group Development

- Environmental Core

- Outdoor Expeditions

- Environmental Investigations

- Commitment to Action

A balanced treatment of these six elements is to be achieved in this course. Each of the elements must be addressed directly each year the course is offered. As students progress from the first year to a second or third year of the course, the relative emphasis on each of the elements will change. In the first year the major emphasis is on course elements at the foundation level; in subsequent years the emphasis is increasingly at the exploration and empowerment levels. 
The six elements work together to develop three strands: outdoor, personal and group development, and environmental. Each strand, in turn, is developed over three levels of experience; a Foundation level, an Exploration level and an Empowerment level. The relationship of these strands and levels to the required course elements is illustrated in the diagram below and on the following pages.

\section{COMMITMENT \\ TO ACTION}

Students will develop lifestyle strategies that foster contact with the natural world, encourage responsibility for local and global environments and encourage living in harmony with others.

\section{EMPOWERMENT}

\section{OUTDOOR EXPEDITIONS}

Students will demonstrate skill, judgment, confidence and sensitivity in a wide range of environmentally responsible activities in outdoor settings.

\section{ENVIRONMENTAL INVESTIGATIONS}

Students will develop knowledge and skills by investigating the effects of human lifestyles on environments.

\section{EXPLORATION}

\section{OUTDOOR CORE}

Students will demonstrate basic knowledge, skills and attitudes necessary for safe, comfortable, outdoor experiences in all seasons.

\section{PERSONAL AND GROUP DEVELOPMENT}

Students will demonstrate understanding, respect and appreciation for self and others.

\section{ENVIRONMENTAL CORE}

Students will demonstrate awareness and appreciation of living things and understanding of basic ecological processes.

\section{FOUNDATION}




\section{COURSE ARTICULATION}

The Environmental and Outdoor Education (EOE) course of studies combines outdoor, personal and group development and environmental strands. Three levels of experience exist within the framework: Foundation, Exploration and Empowerment. Each strand begins at the Foundation level and culminates in the Empowerment level.

LEVELS OF EXPERIENCE

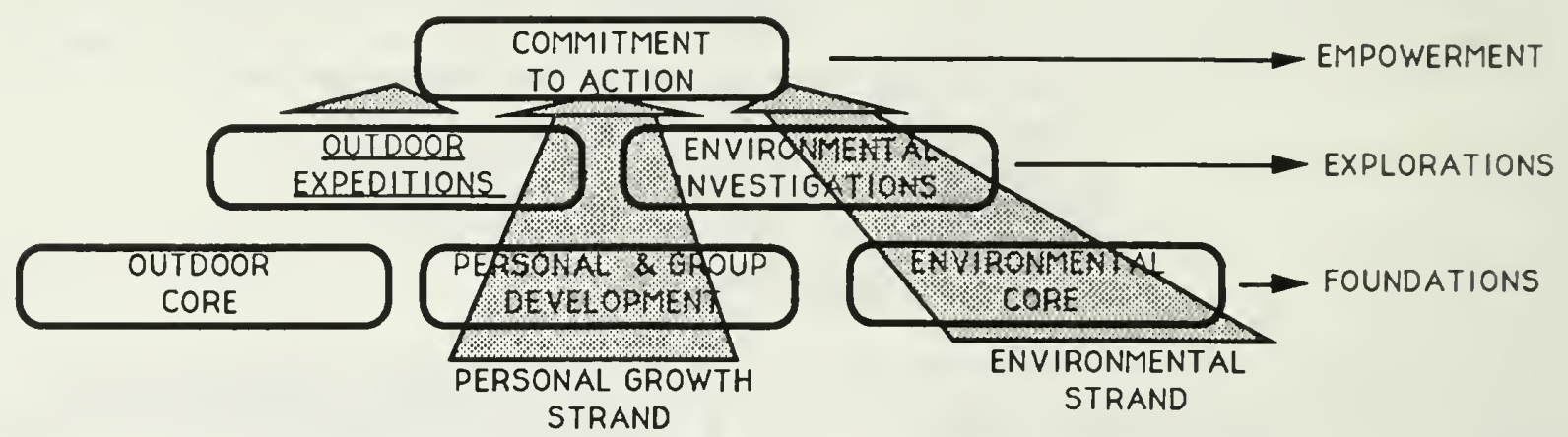

The three levels of experience represent three stages of an experiential education process: assimilation, application and integration. At the Foundation level of experience students assimilate the basic knowledge, skills and attitudes that will preface learning in the later levels. The Exploration level of experience offers students the opportunity to apply the knowledge, skills and attitudes acquired at the Foundation level to real-life situations. In the final level of the framework students incorporate their school experiences into their lives. Each strand incorporates this process of assimilation, application and integration into its development.

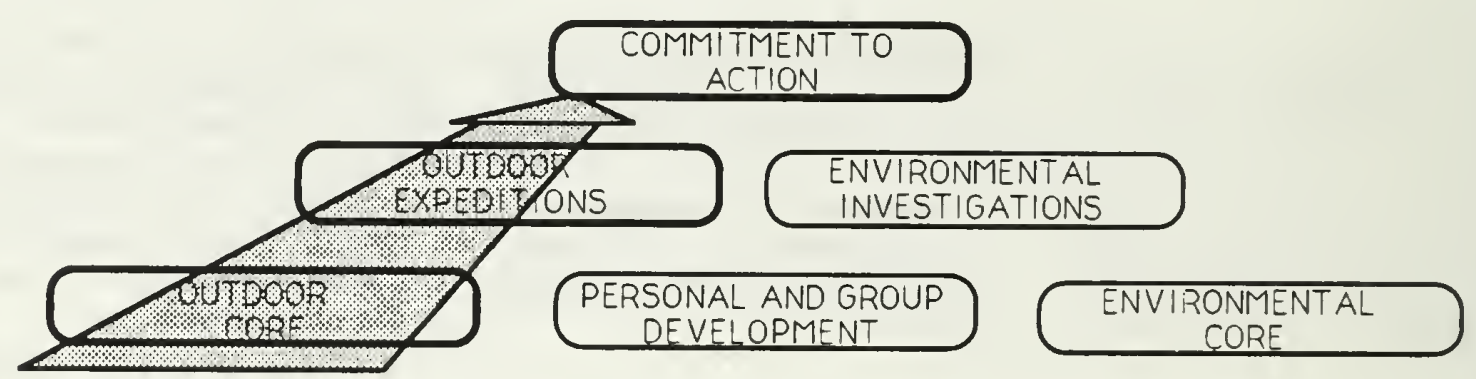

The outdoor strand begins by establishing a foundation of knowledge, skills and attitudes through the Outdoor Core. Before any field trip, no matter how short, students must have the basic concepts, skills and attitudes to ensure that the trip will be a safe, enjoyable one. The Outdoor Expeditions element of the model provides students with the opportunity to apply many of the skills acquired in the Outdoor Core. This strand culminates in Commitment To Action where students identify and act on plans to incorporate outdoor experiences into their lifestyles.

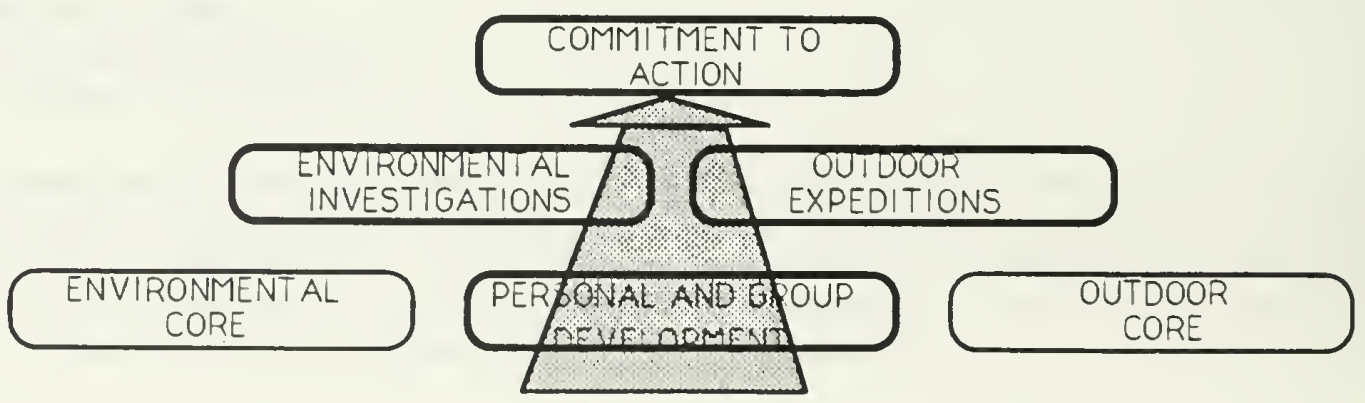


The personal and group development strand follows a similar pattern to the outdoor strand. At the Foundation level of experience the Personal and Group Development element establishes basic awareness and understanding of self and groups. At the Exploration level students will have an opportunity to apply this self and group understanding to problem solving in both the Environmental Investigations and Outdoor Expeditions elements. In the Commitment to Action element, students will build on, or develop as part of their personal growth, plans identified in Exploration.

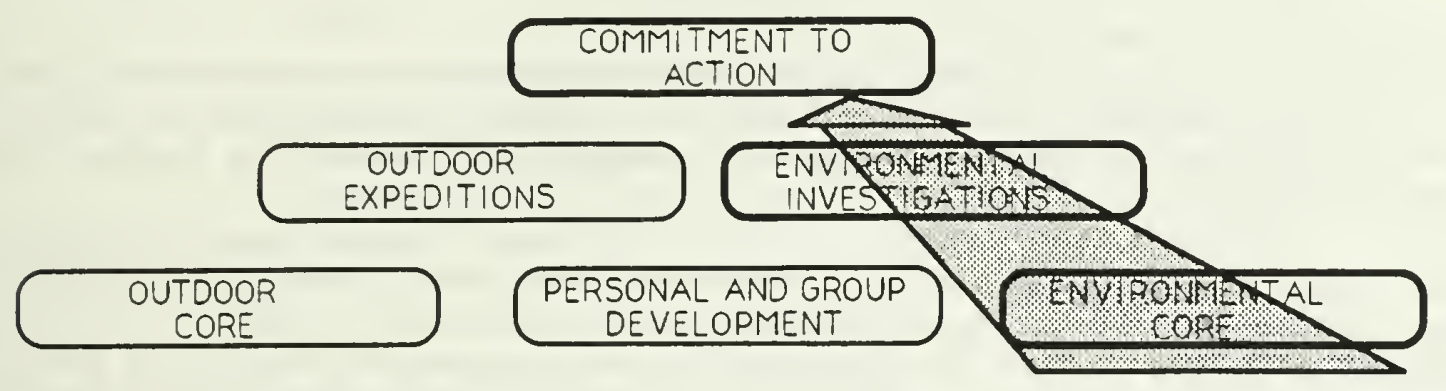

The environmental strand begins with the introduction of material from the Environmental Core. Here, students establish a basic awareness and appreciation of local environments and an understanding of the basic ecological principles that apply generally to all environments. Building on this foundation, students are then introduced to Environmental Investigations. In this part of the course, students will apply problem-solving skills to specific environmental investigations. Students investigate current practices and the alternatives, disadvantages and consequences of each, and decide on the best option. At the Empowerment level, students work toward a personal Commitment To Action. Here, students are encouraged to establish a personal plan of action, act on it and evaluate it.

\section{INTEGRATION OF STRANDS}

The integrative nature of the Junior High Environmental and Outdoor Education Course provides for the development of several learner expectations in one activity. For example, the development of environment awareness and appreciation, which is part of the Environmental Core, can be achieved in combination with the Outdoor Expeditions element of the course. Concrete experiences in outdoor skill development can complement the ecological concepts that are required as part of the Environmental Core. The Outdoor Expeditions element further complements the environmental strand by providing students with the opportunity to see concrete examples of the effects of humans on environments. The outdoor strand supports the environmental strand by providing students with basic self-care skills that enable them to appreciate their environment rather than being preoccupied with the cold or lack of food.

Development of the Empowerment level of the course grows out of previous work in Exploration and Foundation. As a culmination of the outdoor strand, the course encourages students to maintain their skills so that they can continue having contact with the natural world. Such contact leads students to greater awareness and appreciation of their environment, thereby increasing the likelihood that they will act responsibly on its behalf. The environmental strand leads students to look at the effect that their lifestyle has on the environment and leads to positive personal action. Through their awareness and appreciation of environments and their understanding of ecological principles, students can act with sensitivity to environmental concerns in their communities. The personal and group development element asks that students live in harmony with themselves and others. In doing so, their outdoor activities will be enriched and their environmental actions will be more effective. 


\section{REQUIRED/ELECTIVE COMPONENTS}

The course has a required component and an elective component, defined as follows:

- The required component encompasses the knowledge, skills and attitudes that all students should be expected to acquire. This component of the course will be reflected in learning experiences provided for all students.

- The elective component provides opportunities to adapt and enhance instruction to meet the diverse needs, abilities and interests of individual students. It provides enrichment and additional assistance to individual students as necessary. This component of the course is to be reflected by learning experiences designed and selected for students' individual characteristics.

As the program develops it will undergo a shift from teacher-directed learning in the initial phases to teacher-facilitated activities and student-initiated action. In the first unit students will be dependent on the teacher to provide expertise. For example, the teacher provides the information for awareness and knowledge of Ecological Processes. By examining their values in light of this new awareness and knowledge students are more likely to move willingly into problem solving and action.

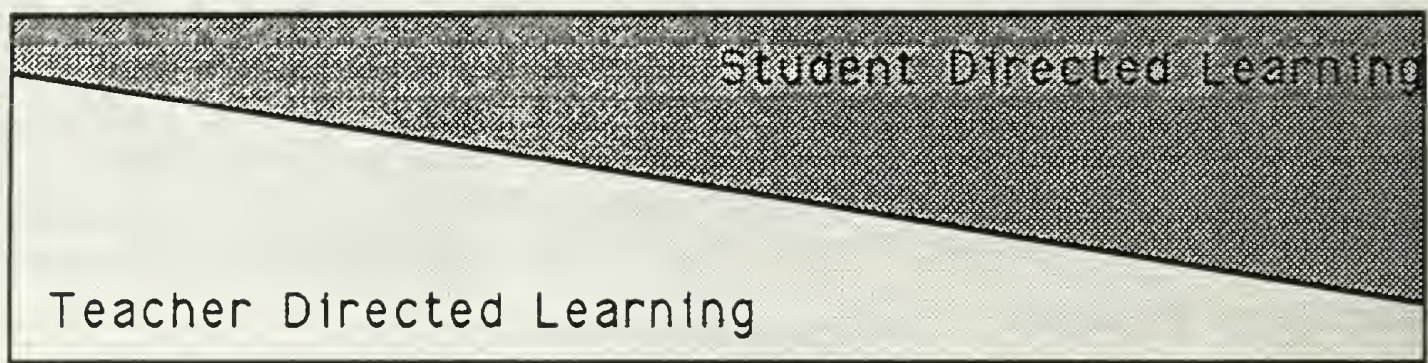

\section{Duration of Course}

The same process of students assuming greater responsibility during a unit should be applied to a fullyear program and to a three-year course of studies. The teacher will be the best judge of how much responsibility classes or individual students can assume. By giving students increased responsibility as they move through the environmental, personal growth and outdoor strands the likelihood of students making lifestyle decisions and acting on them will be increased. 


\section{PLANNING CONSIDERATIONS}

\section{ENVIRONMENTAL AND OUTDOOR EDUCATION AS PART OF THE SCHOOL PROGRAM}

The Environmental and Outdoor Education course may be offered in grades 7,8 or 9. Course subject matter may also be as a three-year course sequence. Where the course is offered over multiple levels, the learning outcomes for each course remain the same, but there will be differences in the level of expectations, the outdoor pursuits selected for study and the environments selected for study.

The number of levels over which the course is offered may be established according to student demand for the course. In cases where there is only a small demand spread over several grade levels, a combined class with students from all three grades could be the basis of organization. Where such a multigrade class involves students who may enrol in the course again at a later level, the teacher will need to design a flexible course to meet the needs of all students. Implementation of a student leadership program may be helpful in providing challenge for the more advanced students.

The nature of the school timetable and the school philosophy will help determine the length of the periods, the possibility of double classes, scheduling of classes adjacent to lunch break or dismissal, the feasibility of field trips, etc.

\section{COMMUNITY}

The community is a major stakeholder in education and can be an effective partner in the education process as well. The use of community members and resources should be an integral part of the Environmental and Outdoor Education program. Local, provincial and federal governments offer a wide range of services and resources as do local clubs, service groups and institutions. Parents and volunteers can assist teachers on trips to fulfil student/leader ratios.

Teachers should have clear guidelines for selecting resource people and the content they might present. Teachers must ensure that the students have the opportunity to hear a variety of perspectives on any issue. A suggested list of guidelines for resource people who want to make presentations to students are:

1. Appropriateness of Presentation: The presentation must be consistent with the level of the group.

2. Pedagogy: The presentation must display sound educational practice.

3. Fairness of Opportunity: The presentation cannot discriminate against particular populations.

4. Need: The activity should not duplicate what is already taking place.

5. Motive or Hidden Agenda: The purpose and benefits of the activity must be clearly stated.

6. Screening: References and evaluations must be available on request. Auditions and interviews might be conducted

7. Extra-Curricular Involvement: Consideration must be given as to whether the presentation would be best suited as a curricular or extra-curricular activity (e.g. noon hour presentation to entire school).

8. Cost: Time and financial costs must be considered by the presenter and not become a burden for the participants (school and/or students). Any anticipated costs should be disclosed beforehand.

9. Health and Safety: Participants must be aware of and be protected from the hazards related to any activity.

10. Exploitation: Activities must NOT, in any way, take advantage of the students as a captive audience.

The teacher should use as many community resources as possible and letting the community know about plans and specific needs can generate unexpected help. 
Budgets for Environmental and Outdoor Education should be established based on objectives, course requirements, and community and school support. Equipment and transportation are two of the most common logistical and budgetary problems a teacher faces when preparing for a field trip.

Some equipment options available include purchasing, renting, borrowing or constructing equipment. Factors to consider when deciding these options include: availability of capital, maintenance and storage capabilities, proximity of rental facilities, rental fees, loan pools, availability of gear from other organizations, possibility of joint purchases with other organizations, and availability of the resources to design and construct equipment.

Transportation options include busing, parent or volunteer drivers, public transport and student-powered transportation such as cycling. Factors to consider when arranging transportation include board policy on volunteer drivers, costs, availability of transportation and proximity of field trip site.

\section{ENVIRONMENTAL IMPACT}

Environmental impact has many interpretations. The most important factor to consider is the ability of the environment to recuperate from impact. Fragile or high use areas cannot regenerate themselves quickly enough between times of use to maintain themselves in a natural state. Subsequently many environmental codes and rules of conduct have evolved to minimize each individual's effect on the environment. Even in resilient, infrequently visited environments there are still many sound environmental practices that apply. Examples include selecting campsites with care, establishing latrines well away from bodies of water, storing and disposing of foodstuffs properly, and minimizing the scarring of sites by using only deadfall or using one established fire pit.

\section{SAFETY}

Safety is a prime consideration in the selection of learning activities. Students should not be put in situations where they are engaged in activities beyond their skill levels or where adequate instruction support, guidance and supervision are not provided. Assessments must be made both of the skill levels of individuals and of groups in determining appropriate activities. See Chapter 7 for further guidelines with respect to safety.

\section{BALANCED PERSPECTIVE}

In developing the environmental components of this course, students are to be provided learning experiences that reflect a broad understanding of environmental issues and concerns. Presentations of course content in a way that reflects a singular or narrow view of environmental concerns would not be consistent with learning expectations and must be avoided. For clarification of policy in this regard, please refer to Appendix A Policy Statement on Controversial Issues. 


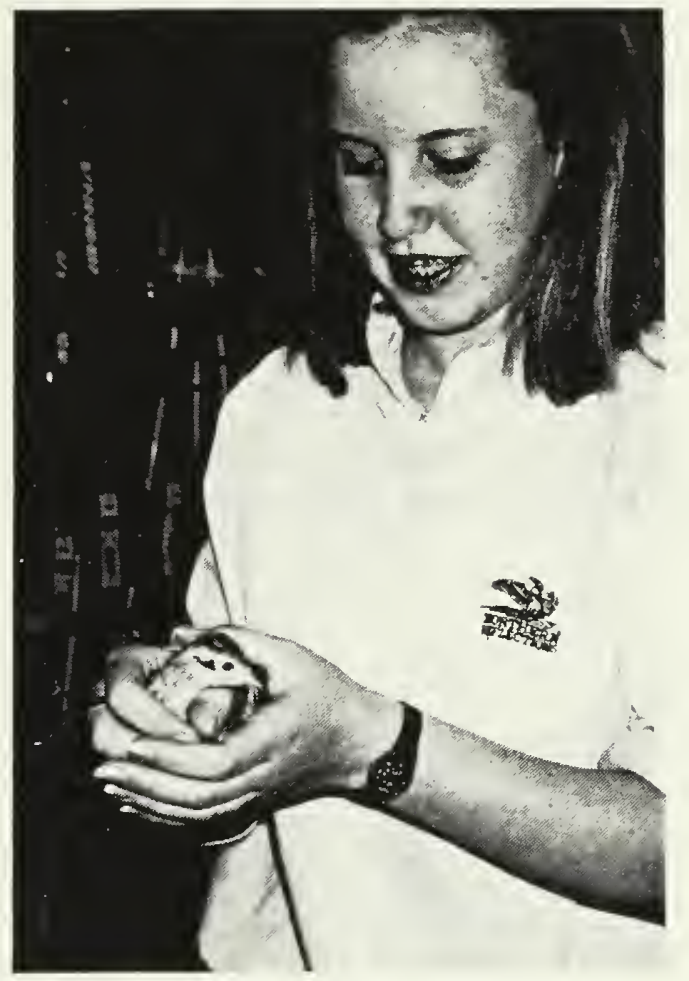

Chapter

\section{Course Content}

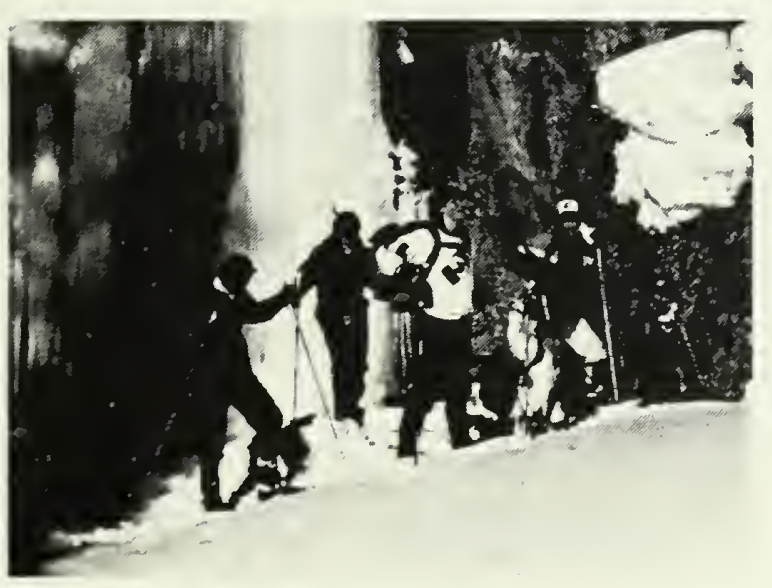




\section{INTRODUCTION}

This chapter includes:

- Specific learner expectations

- Sample student activities

- A list of resources that support identified student activities

Note that the learner expectations are excerpted from the course of studies. These statements identify the mandated content of the course.

Student activities and resource lists are included in this section to help teachers plan the course. Activities and resource lists are provided as service material and should not restrict other approaches that may be used to achieve the learner expectations.

Resources are listed by title only. For further information about these resources see the comprehensive list of resources in Chapter 3. Chapter 3 includes bibliographic information, sources and annotations about the content of each resource. 


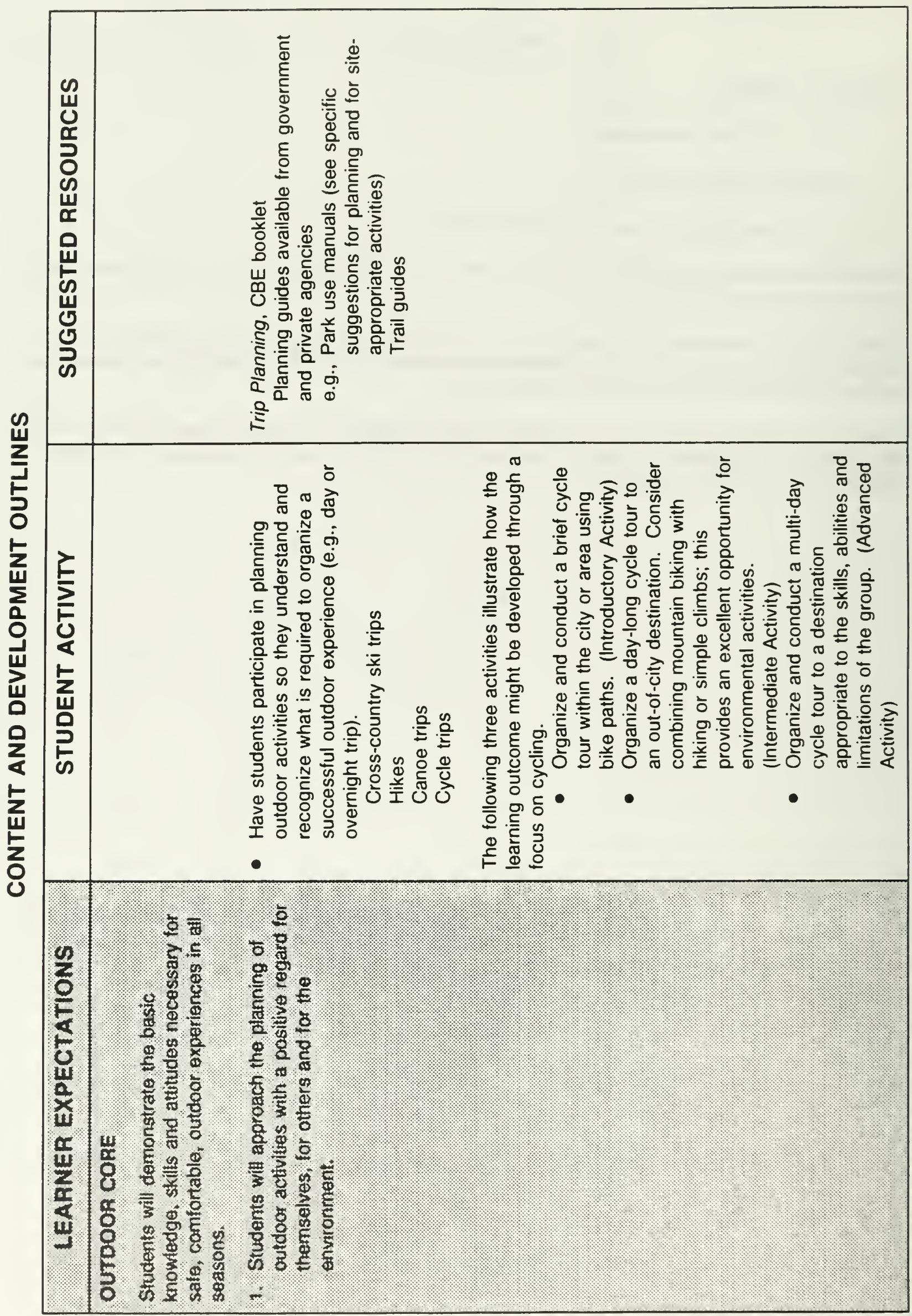




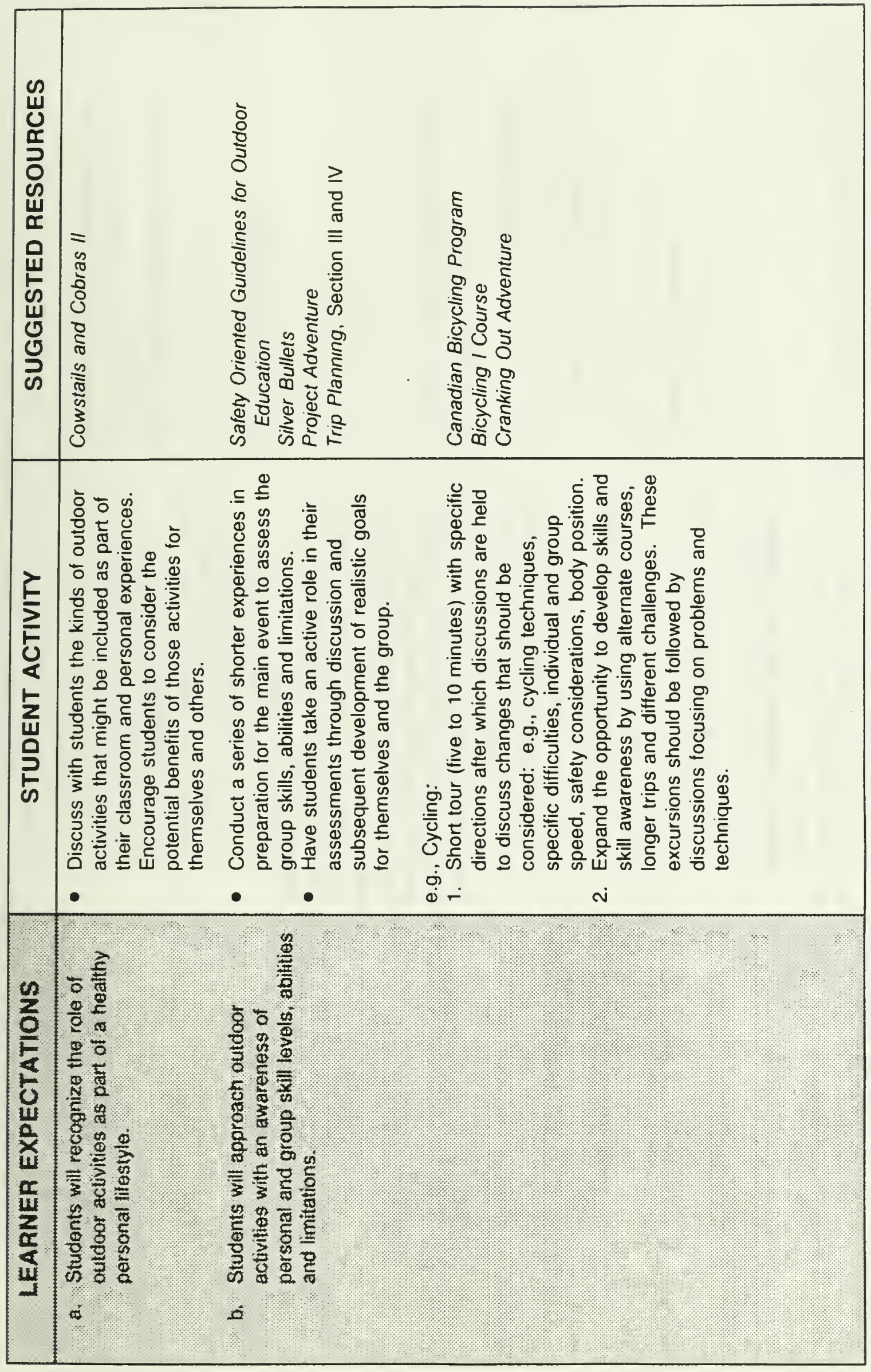




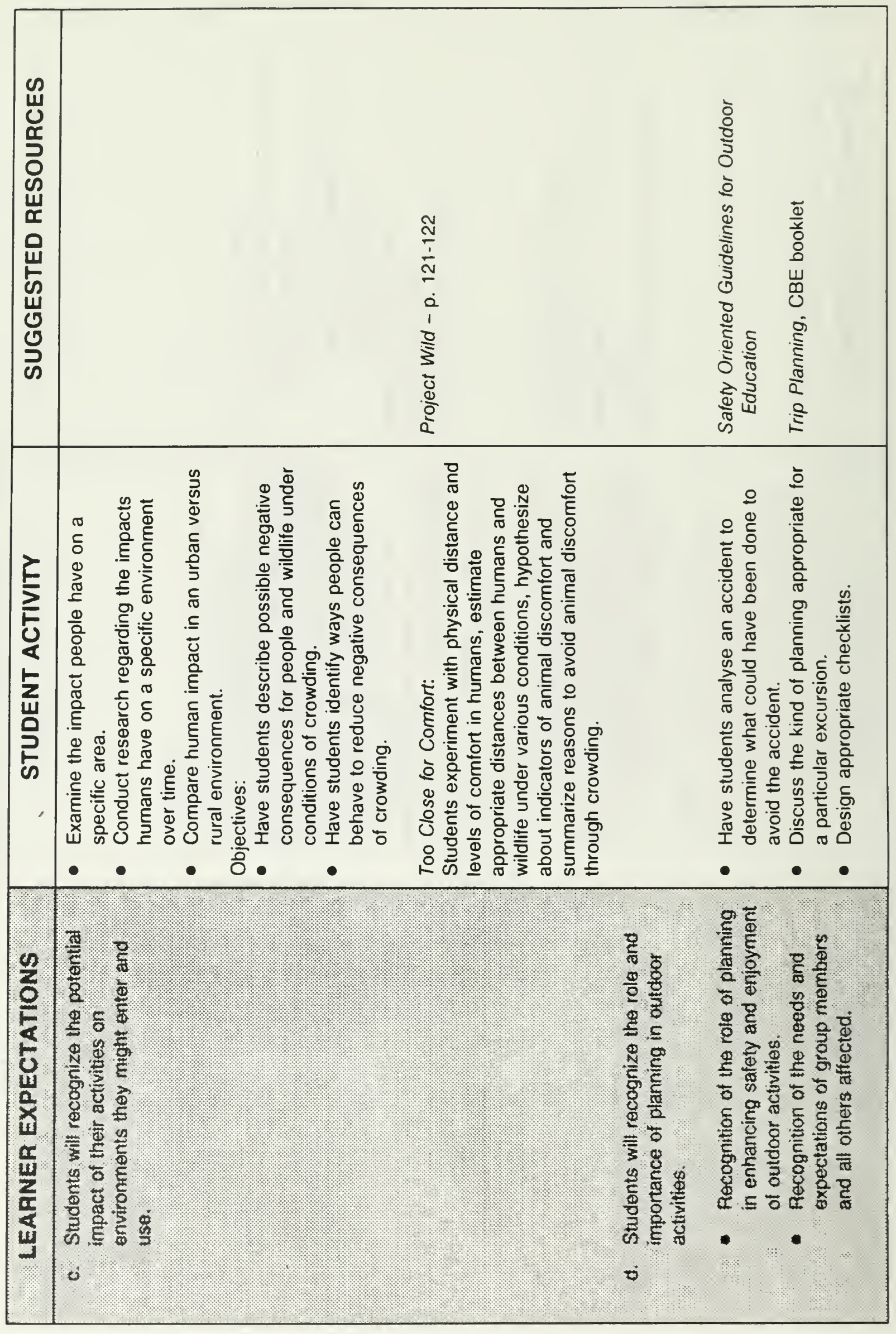




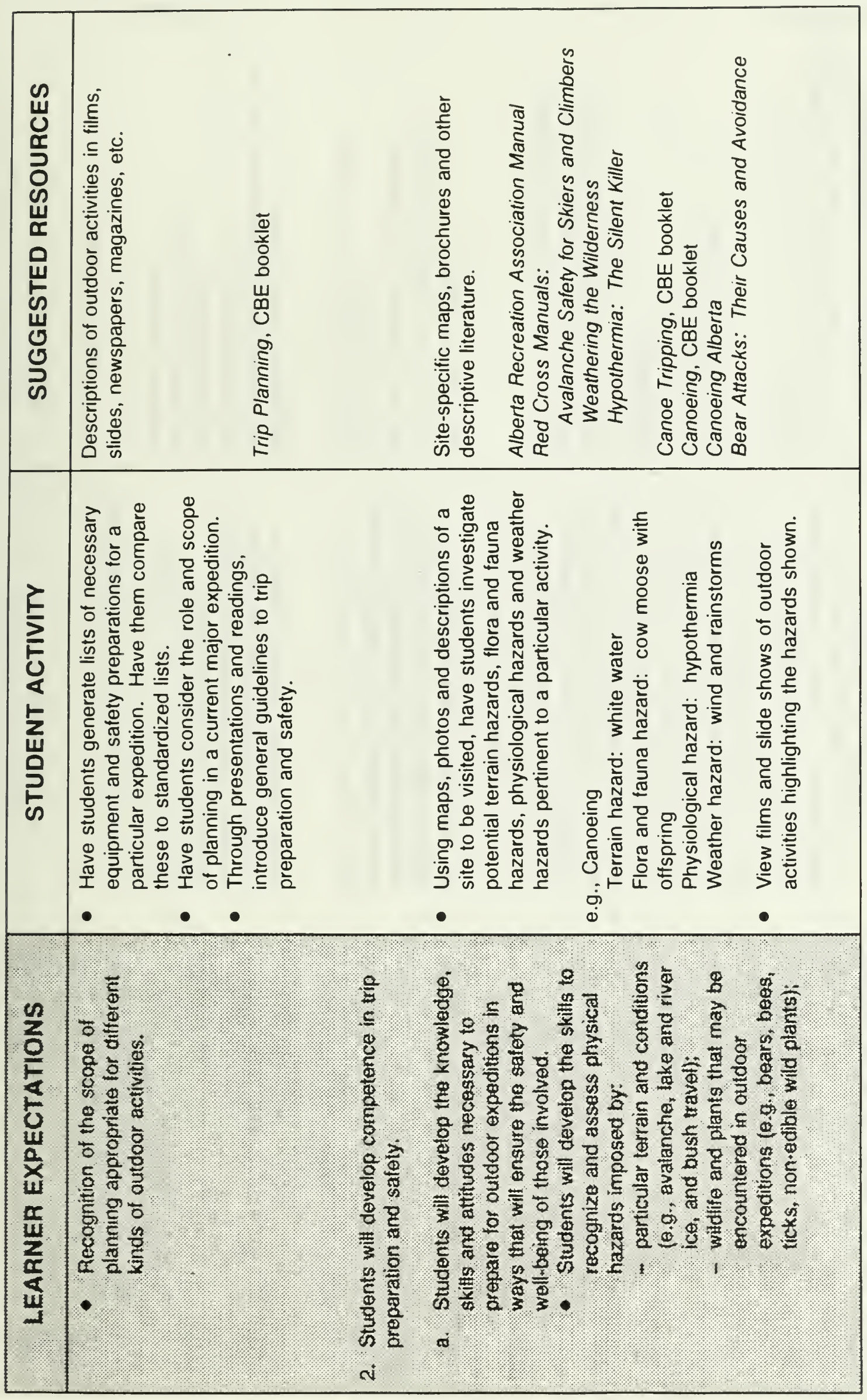




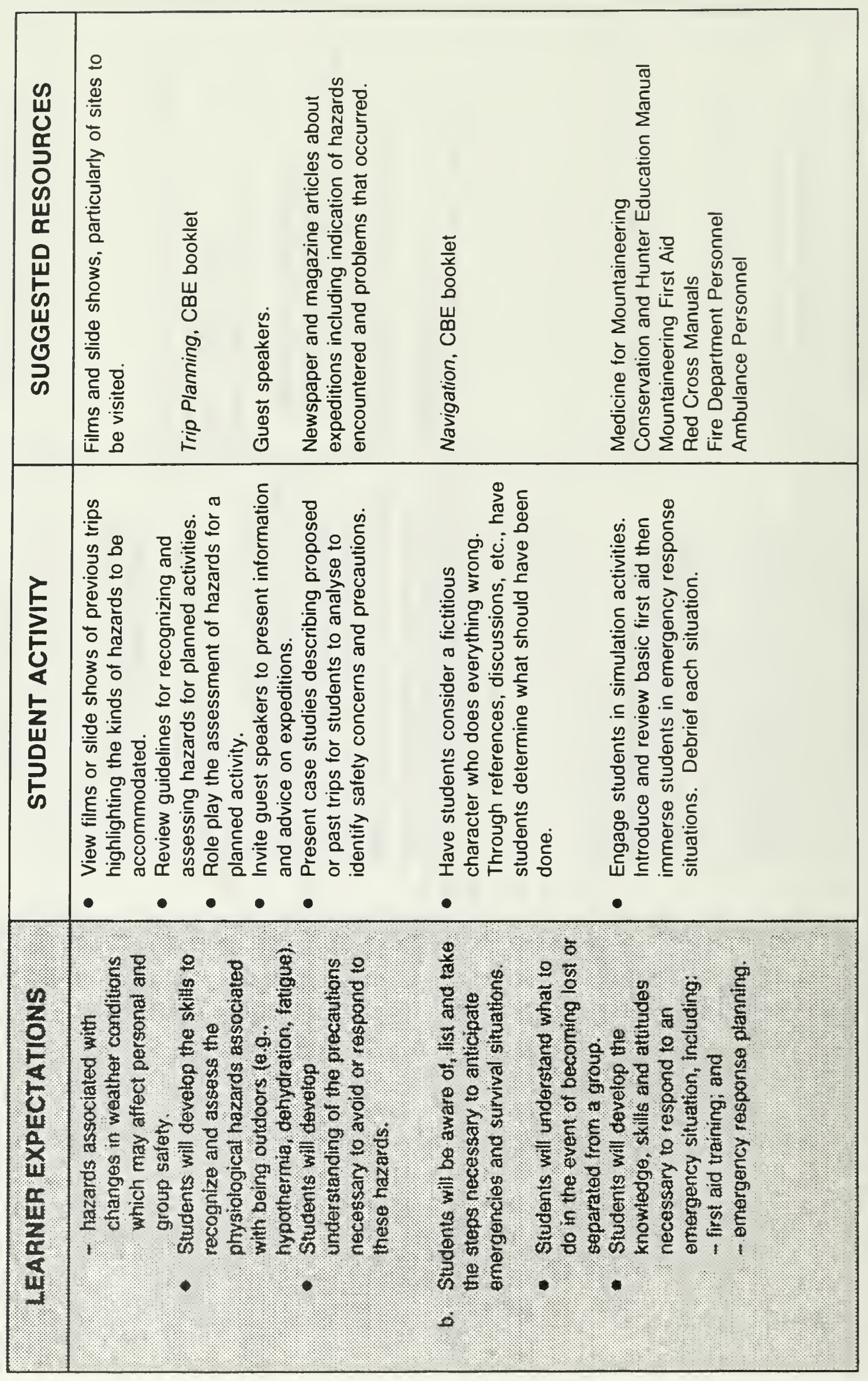




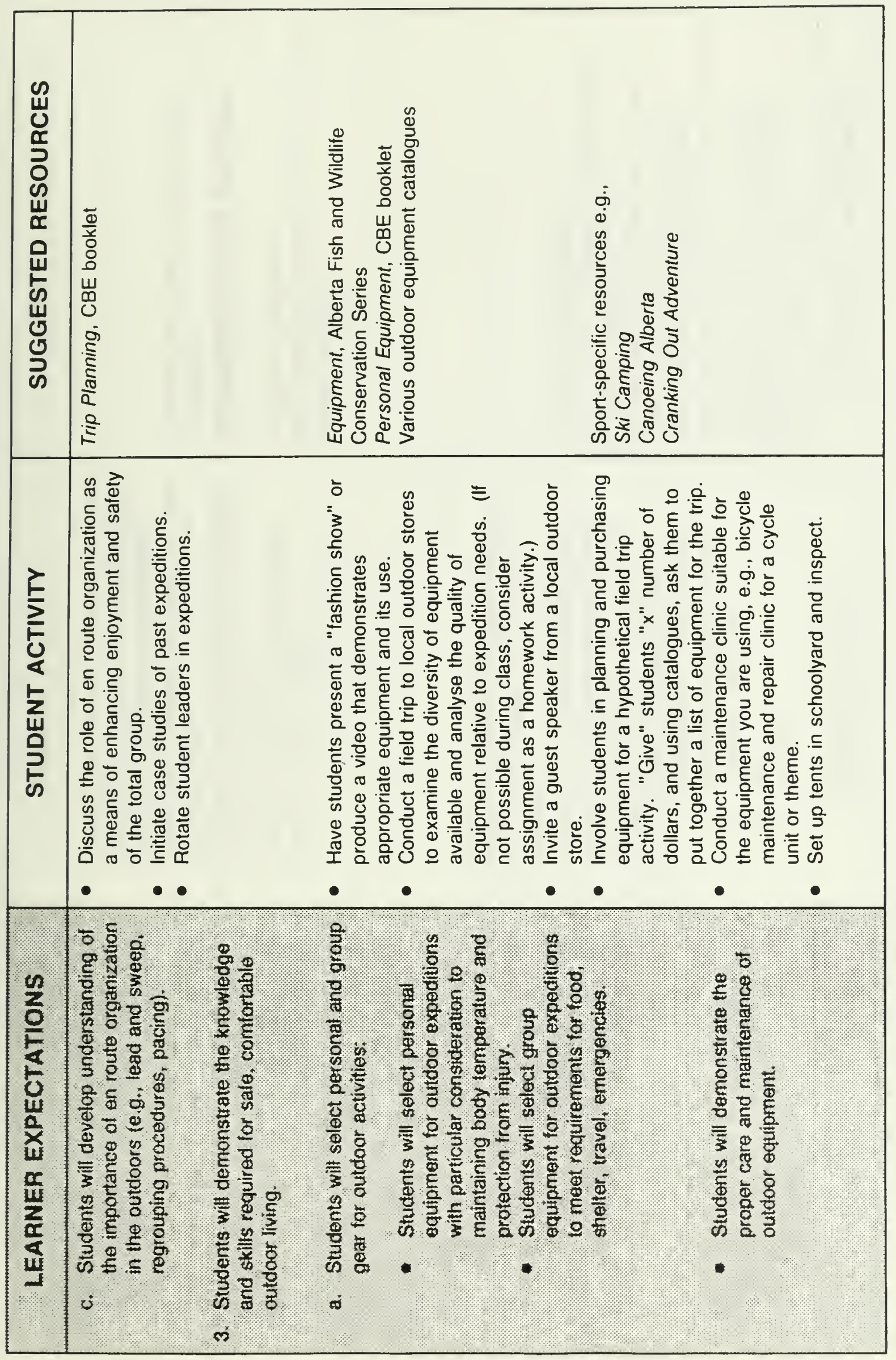




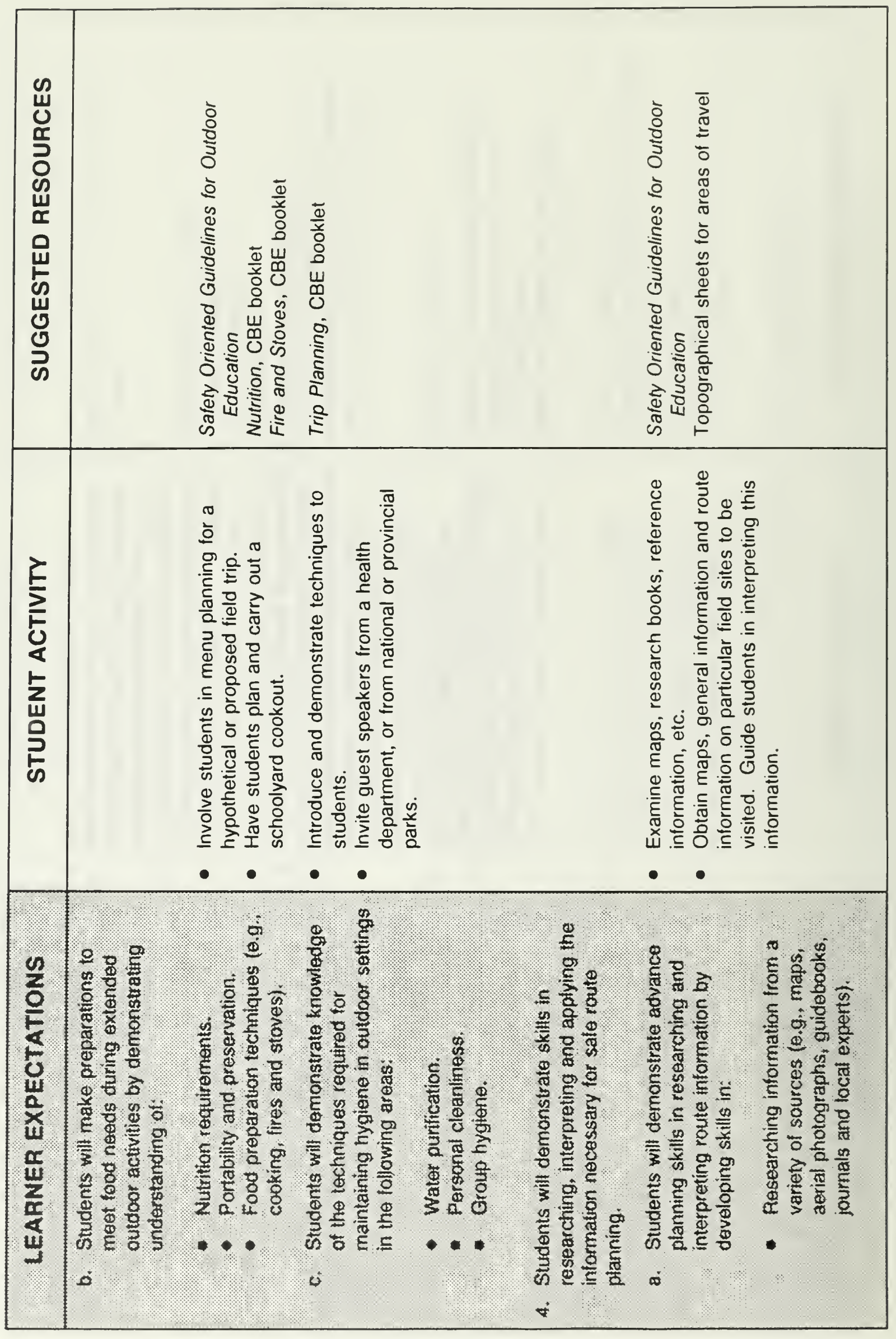




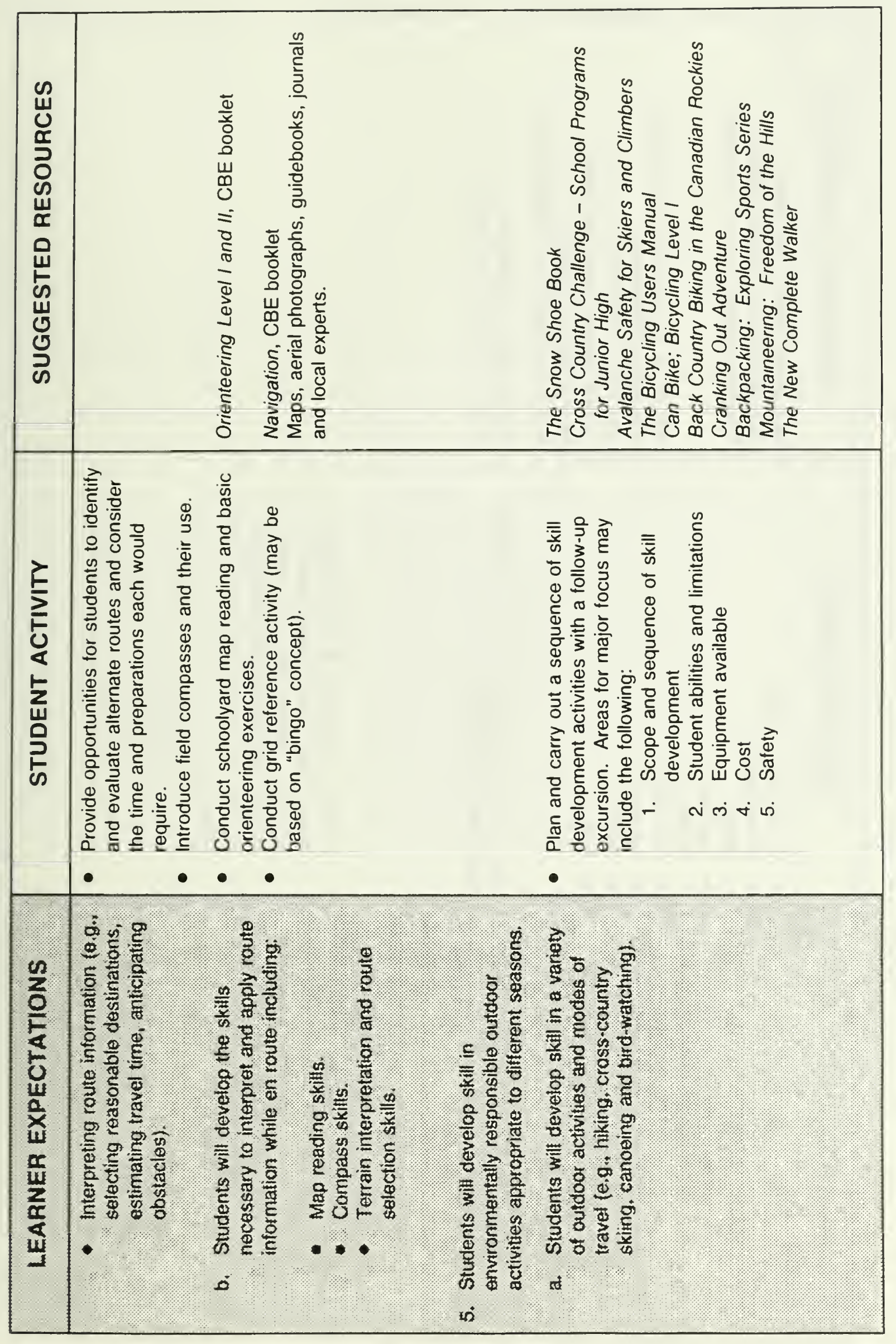




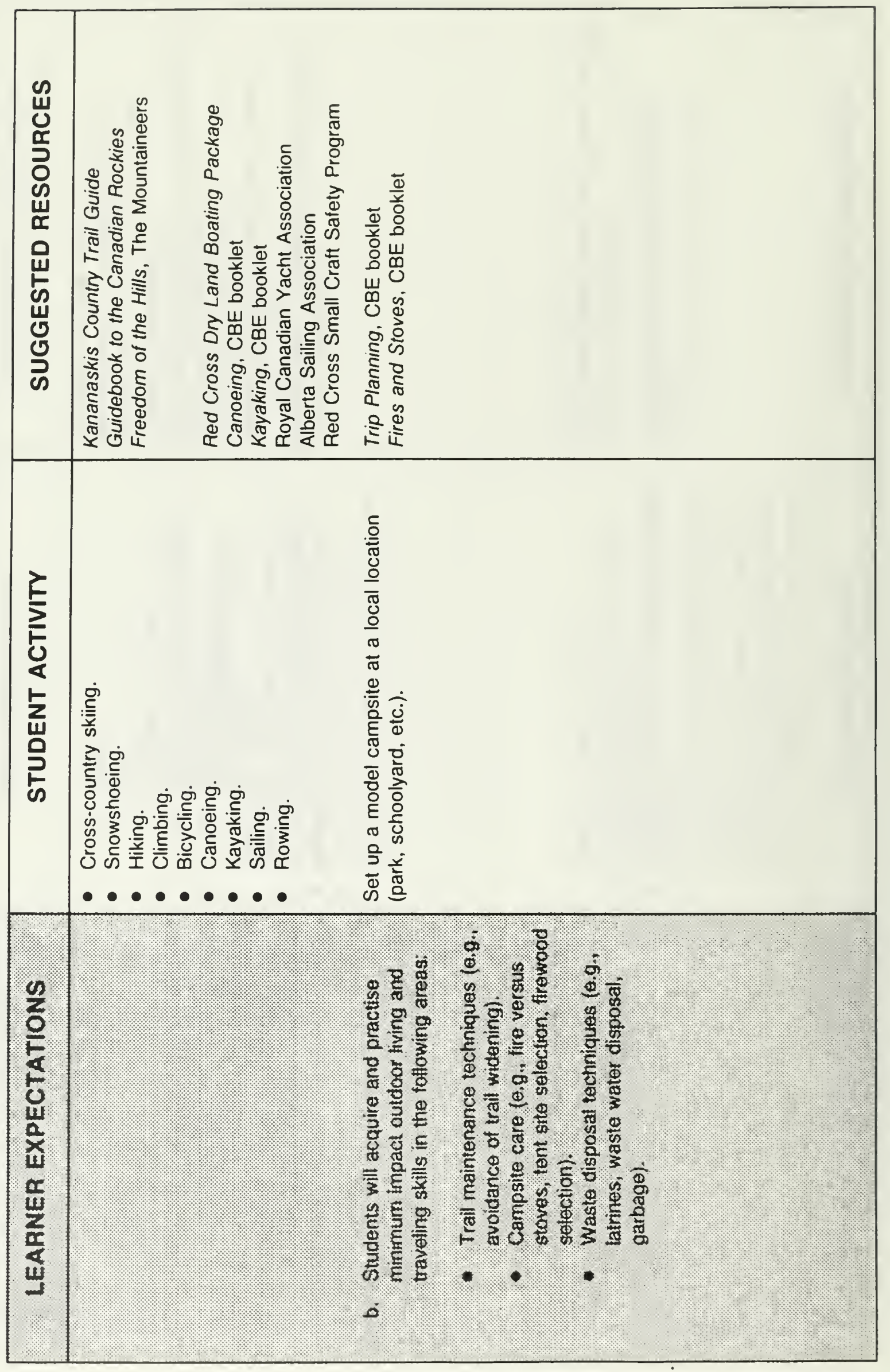




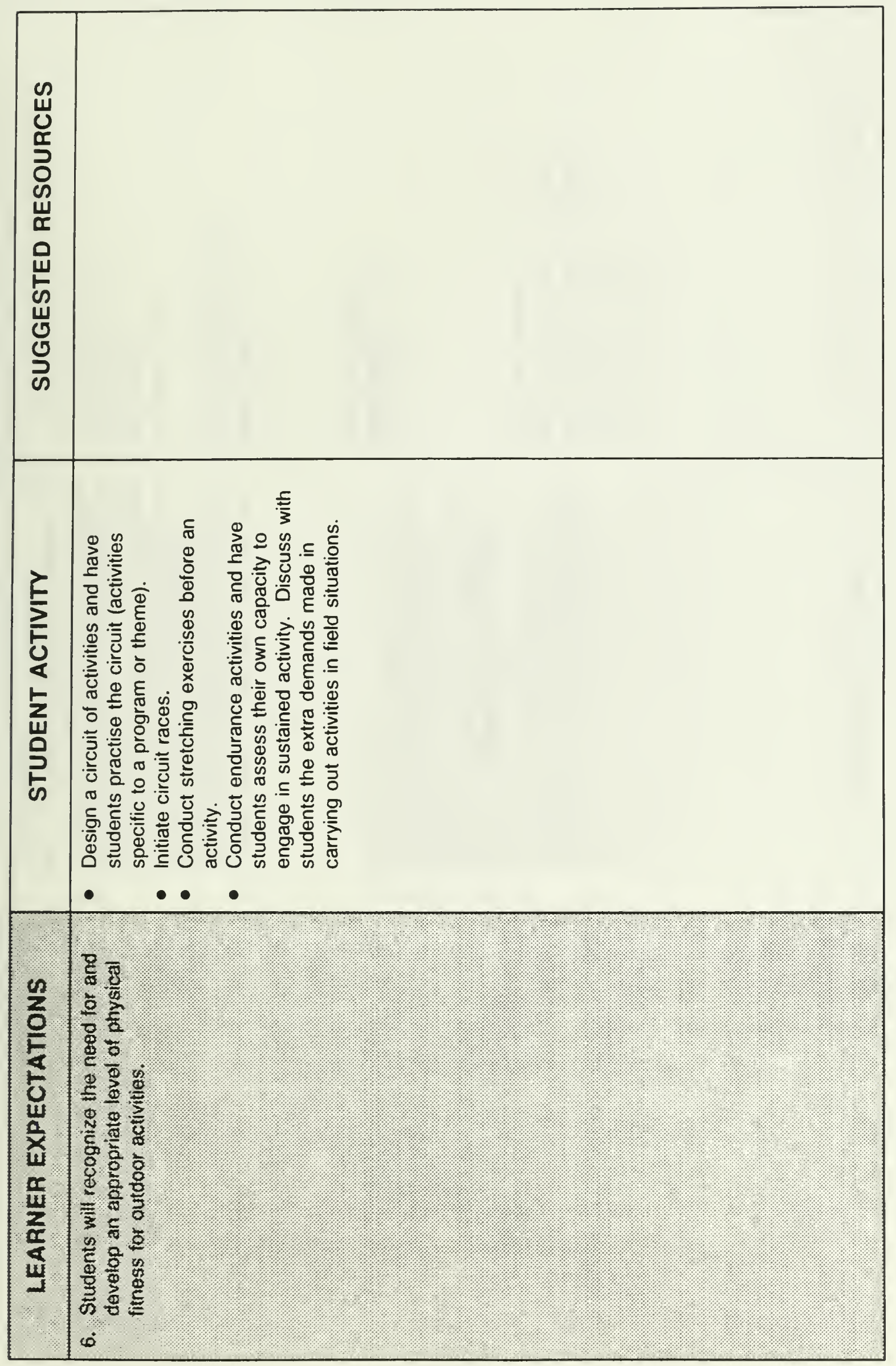




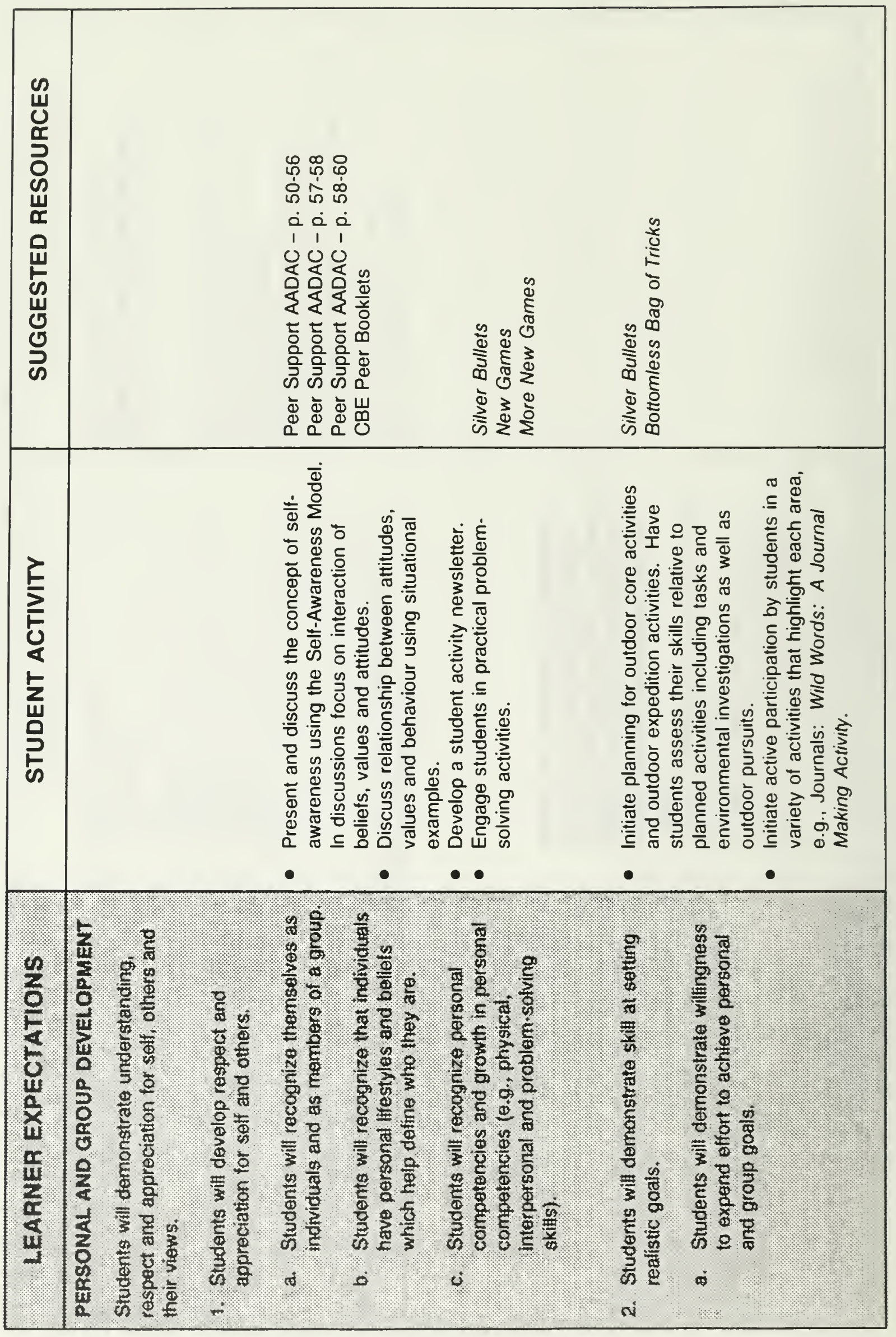




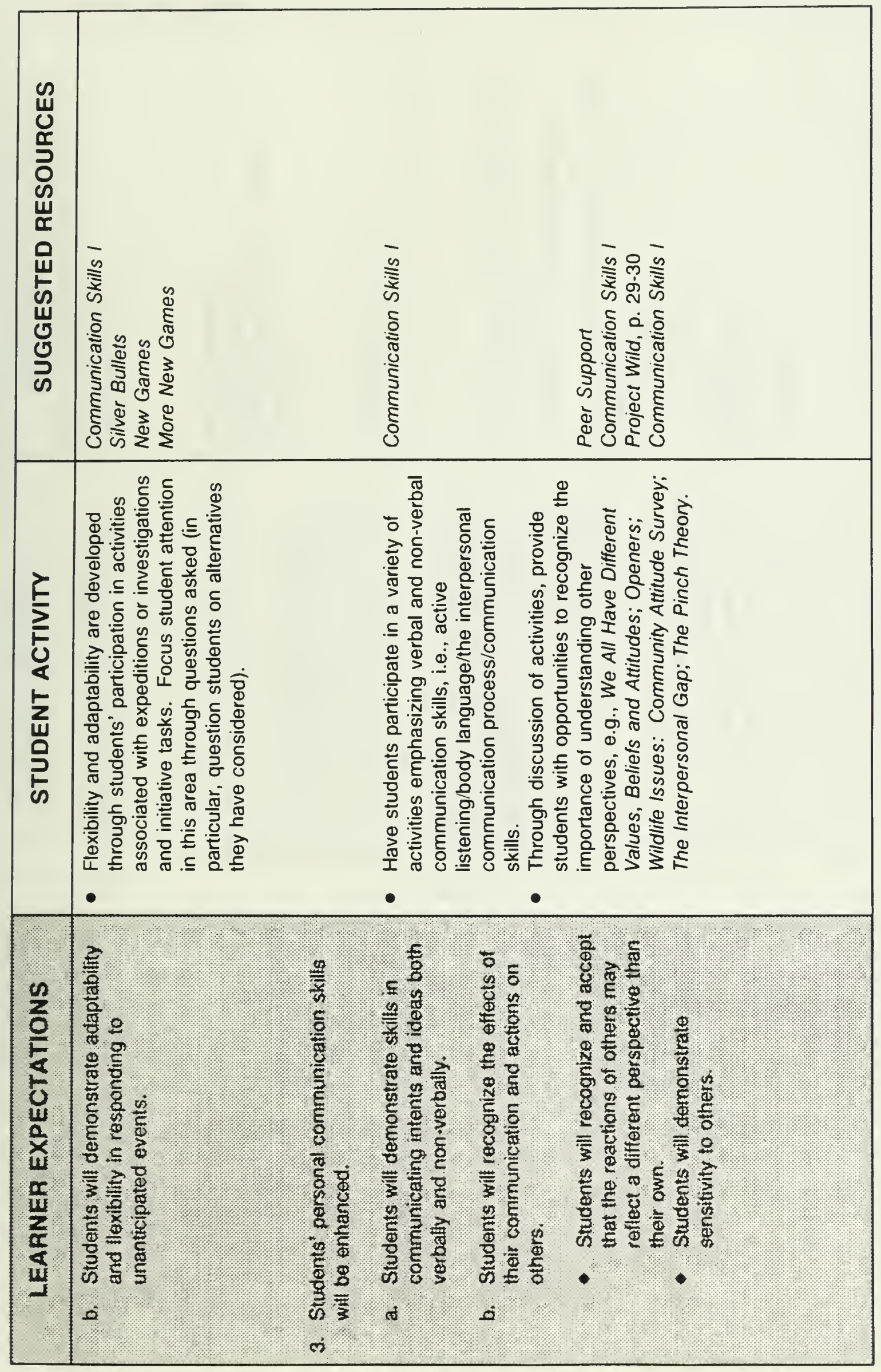




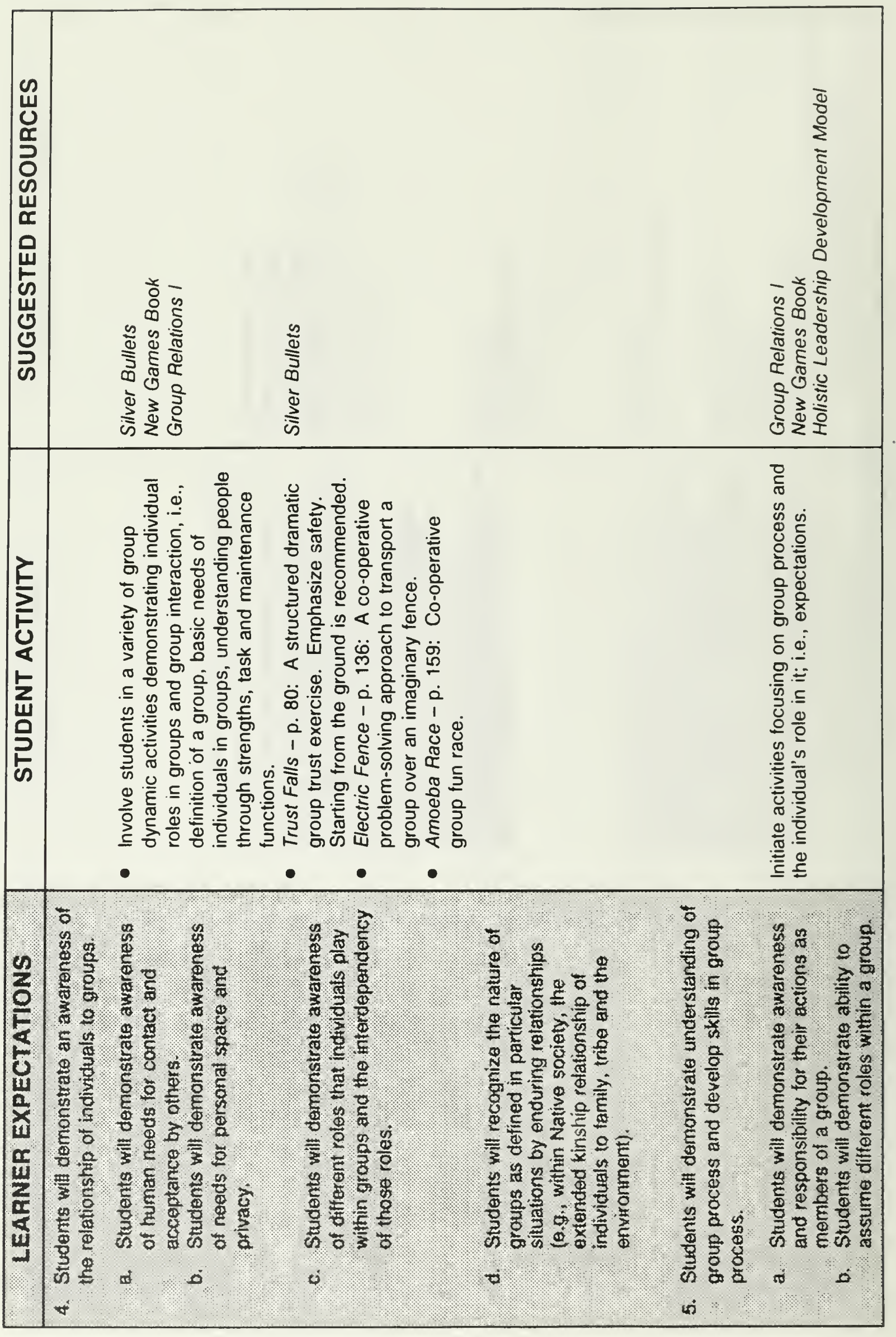




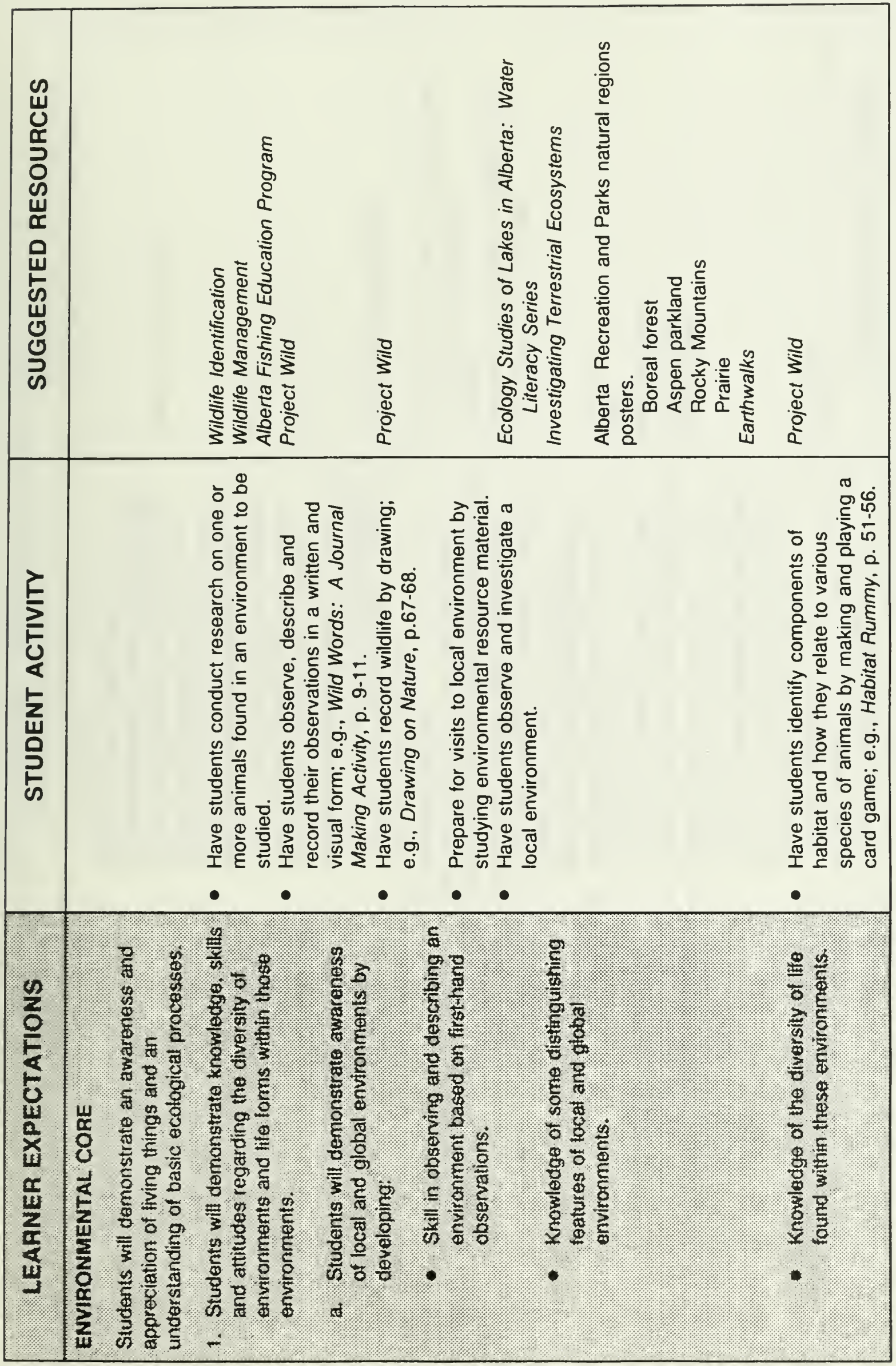




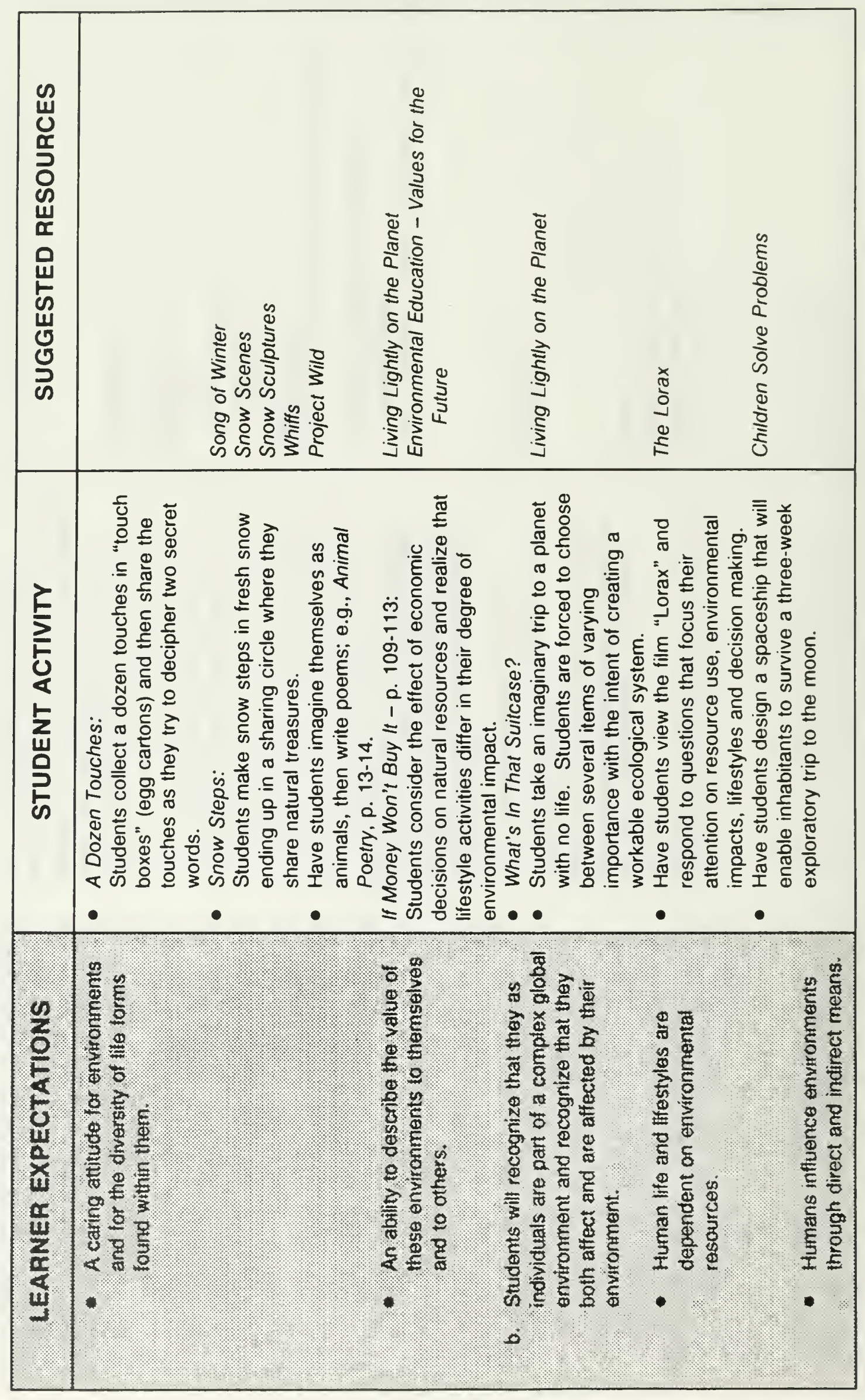




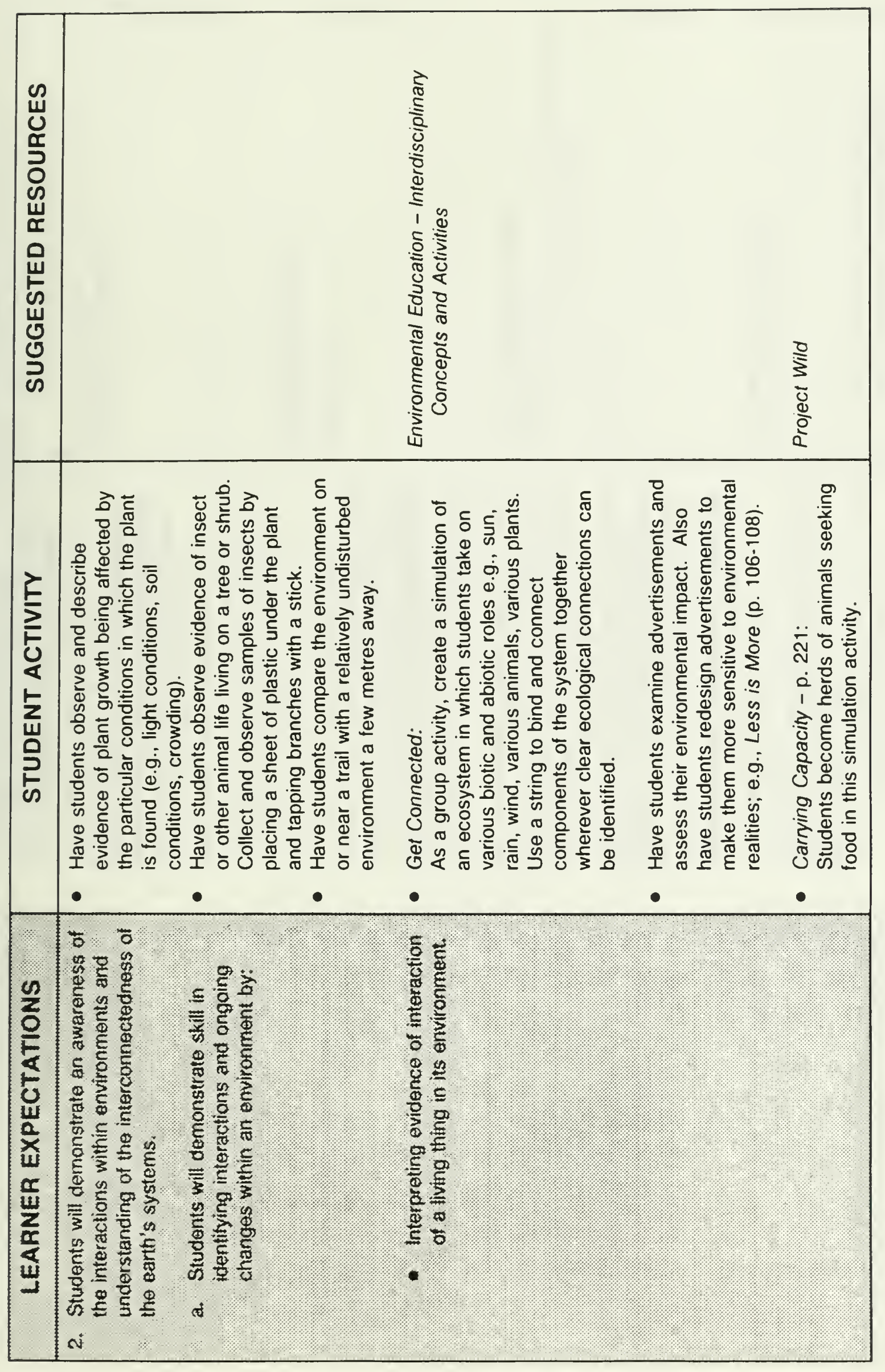




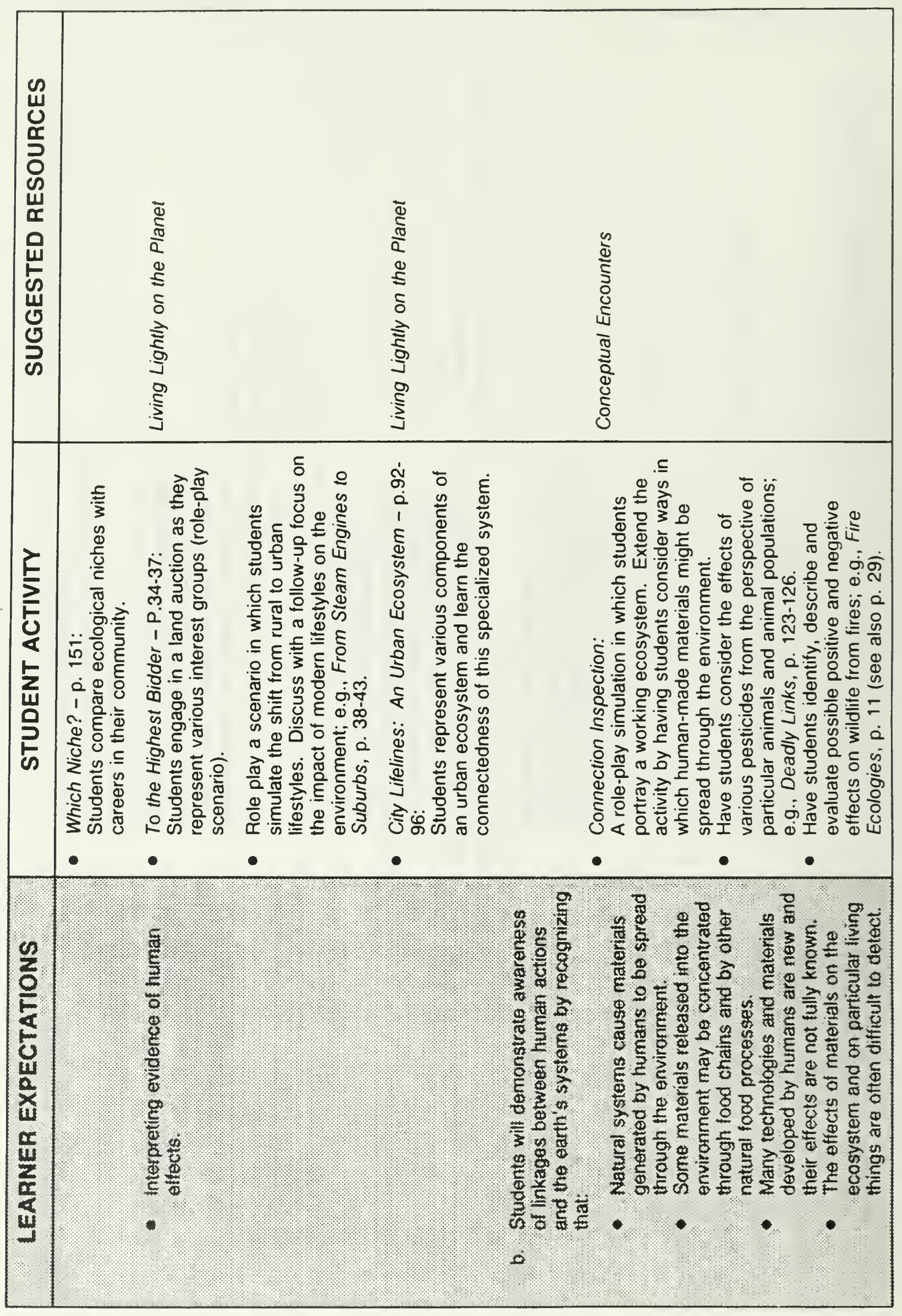




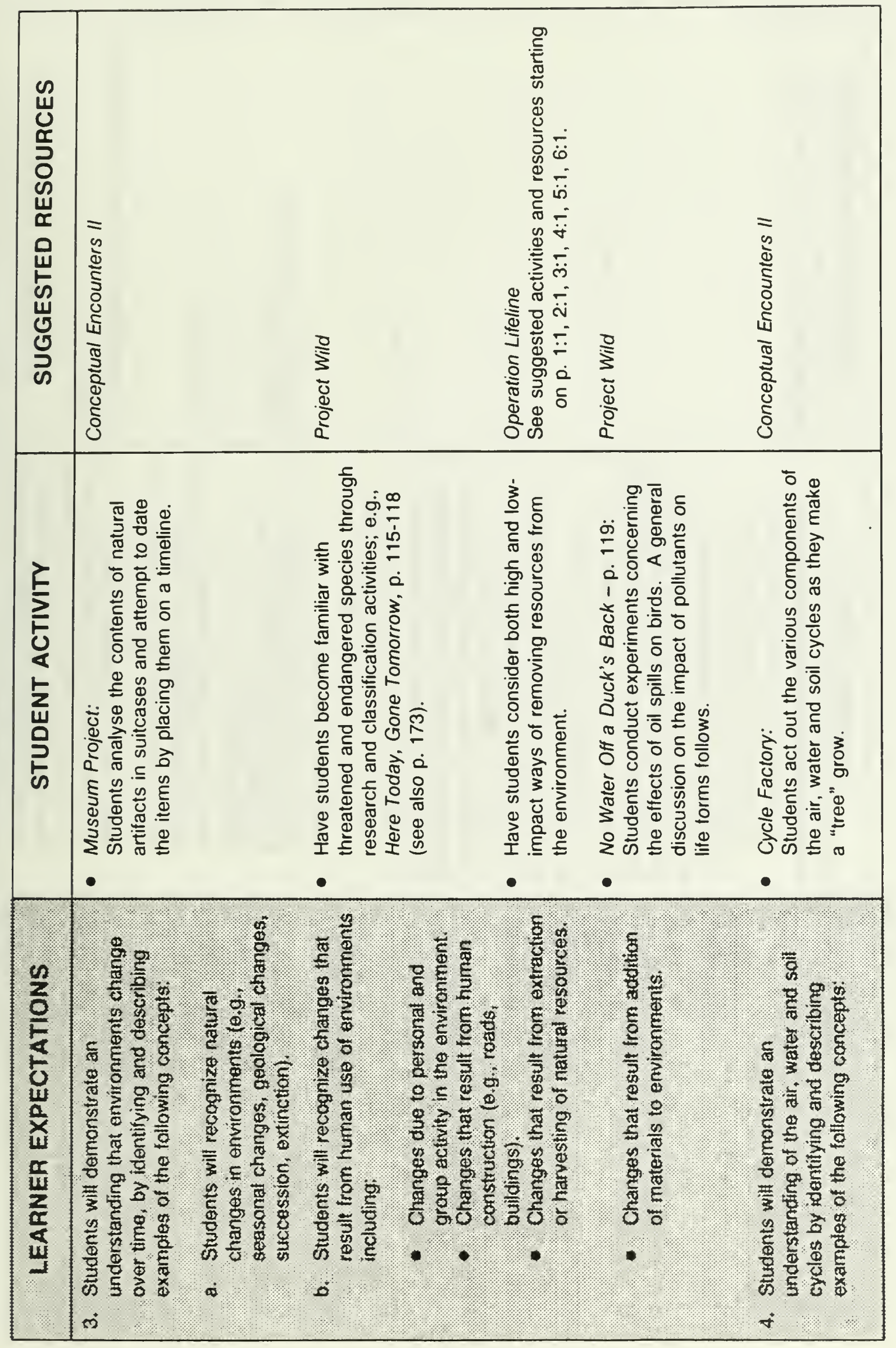




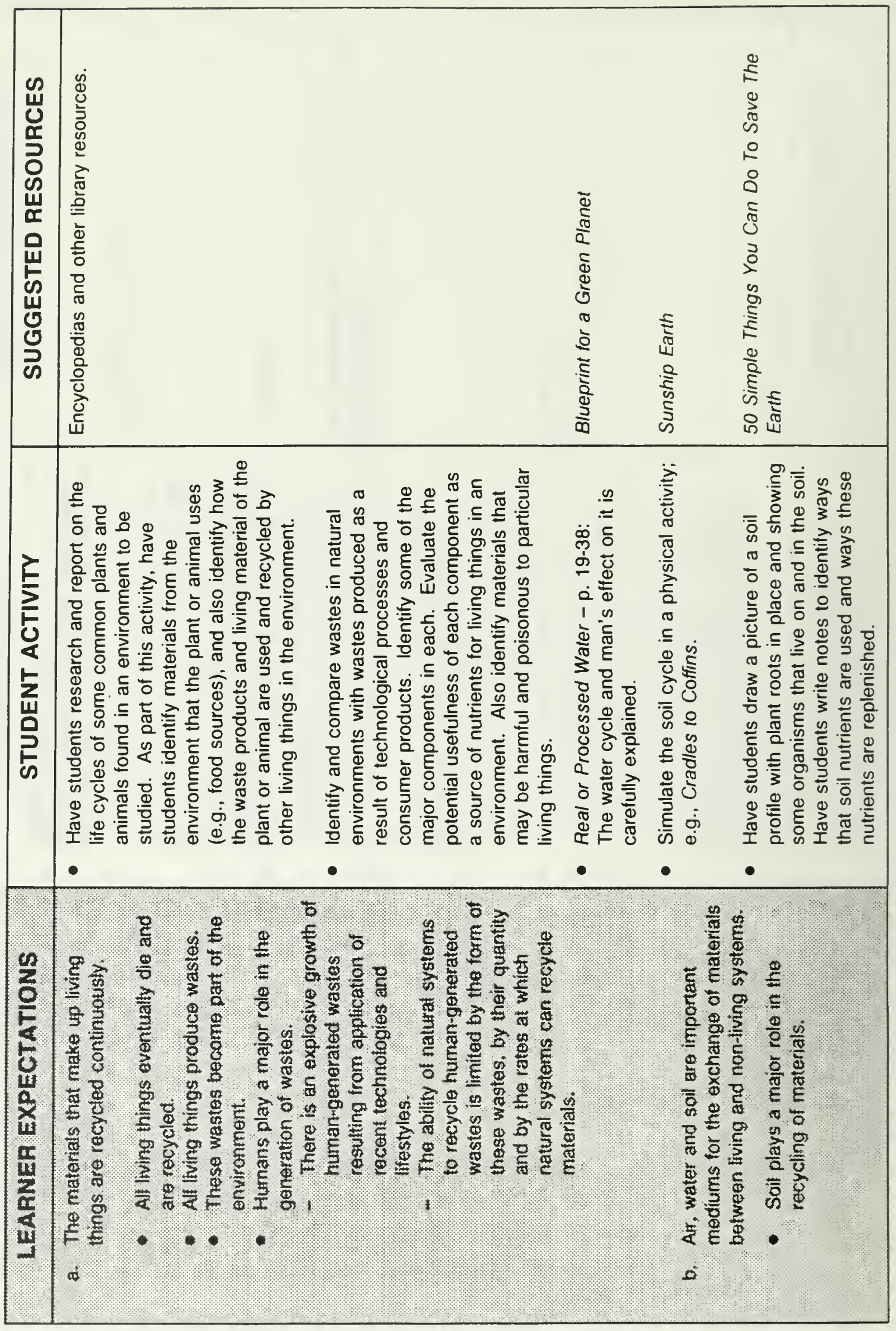




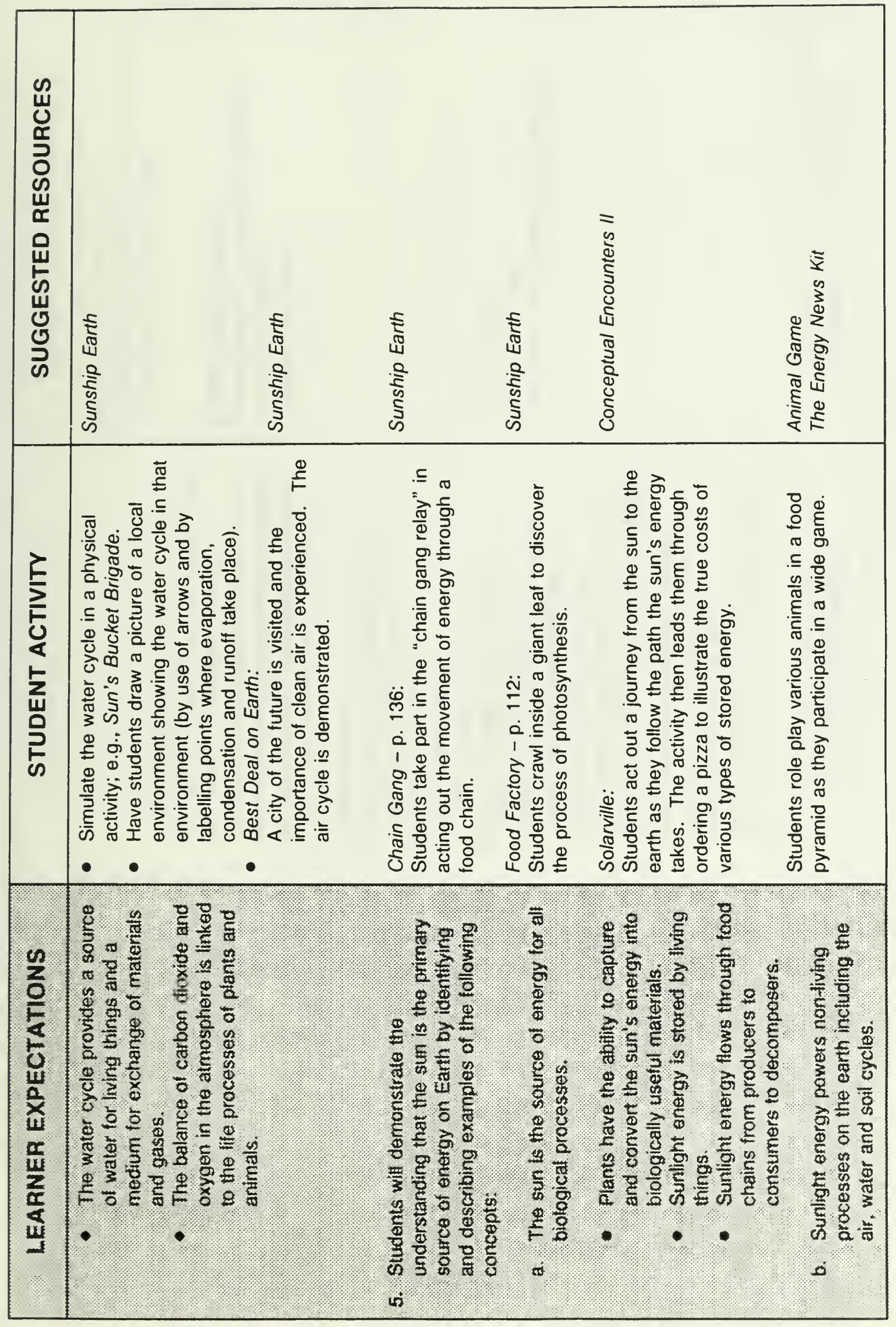




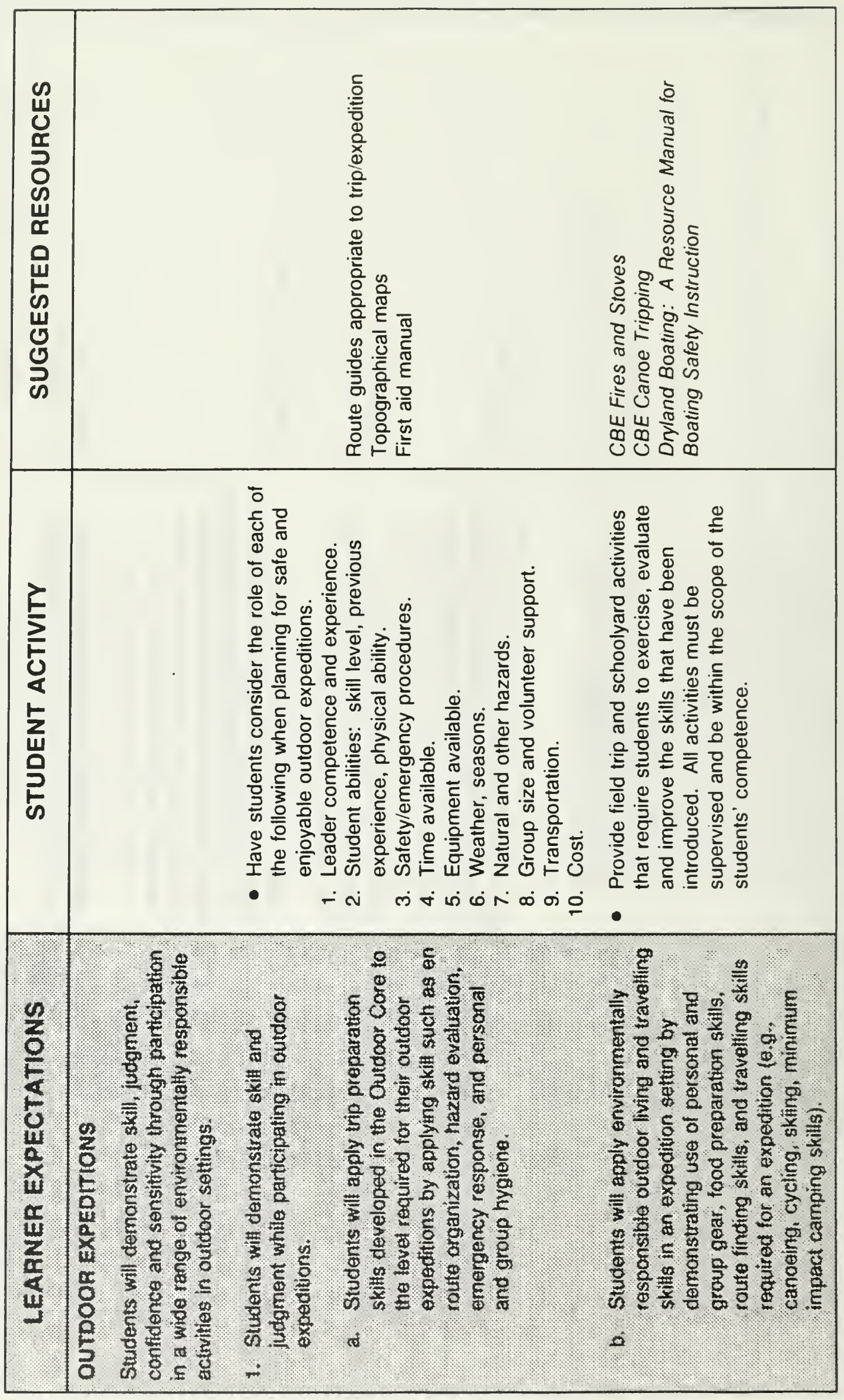




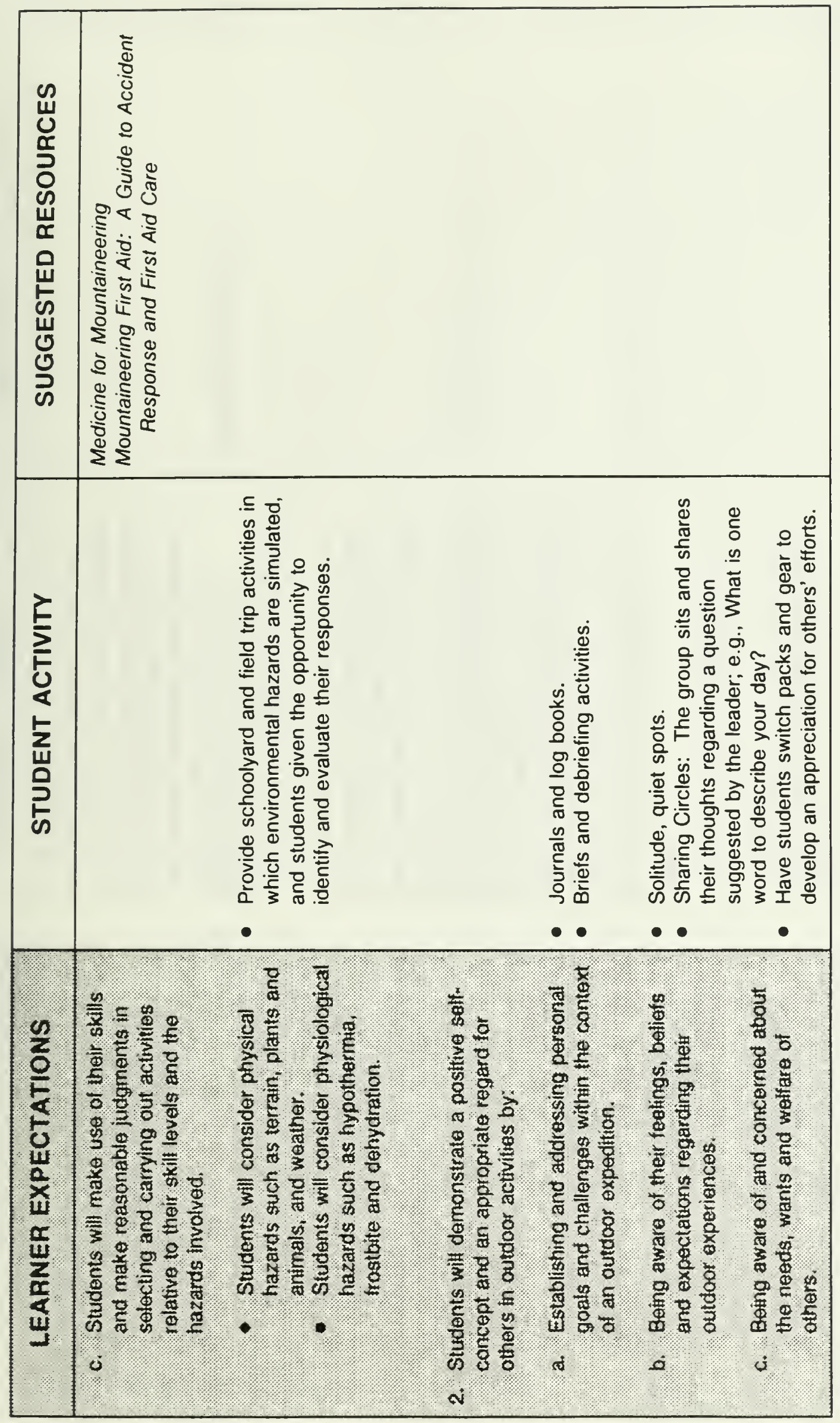




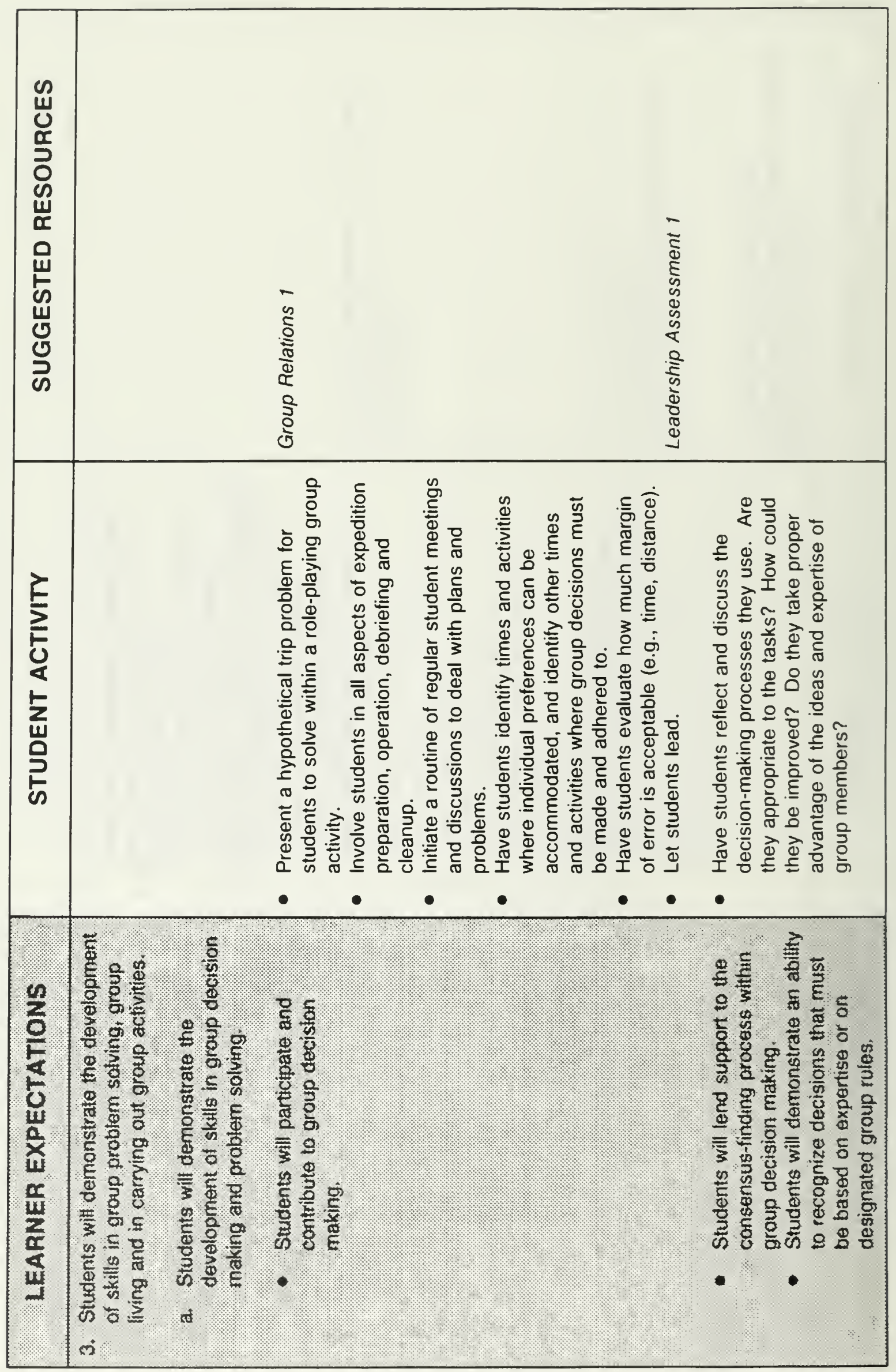




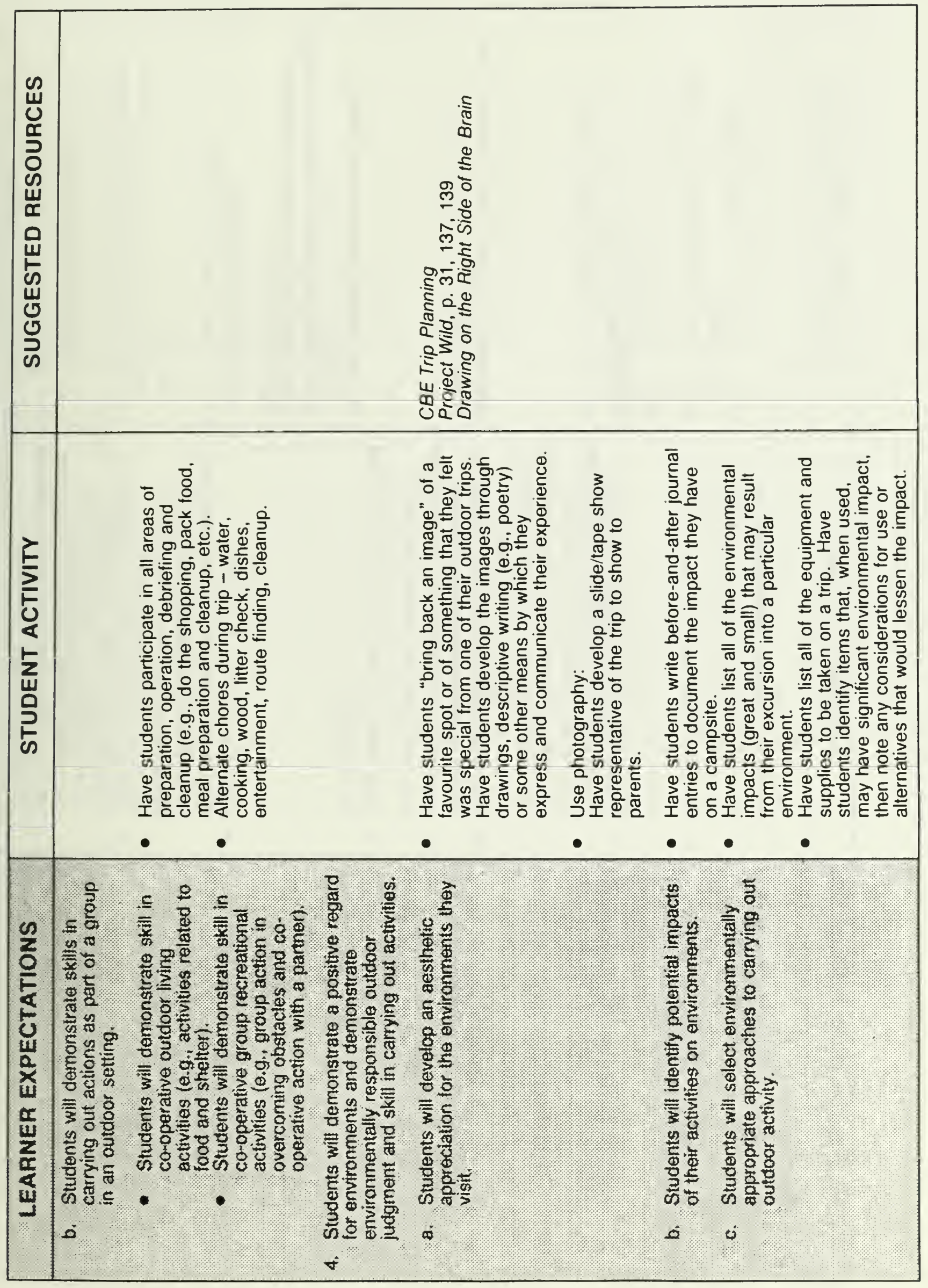




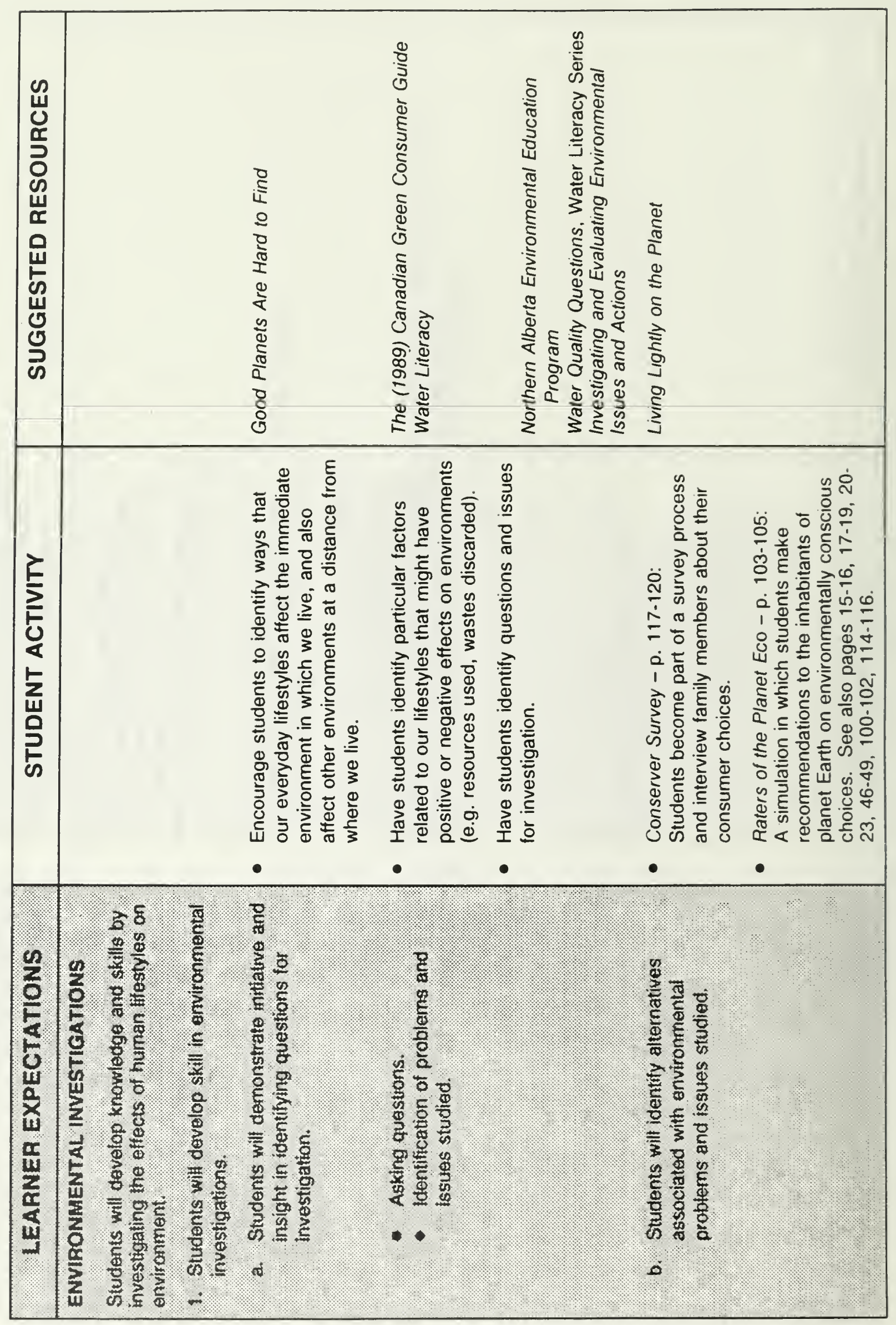




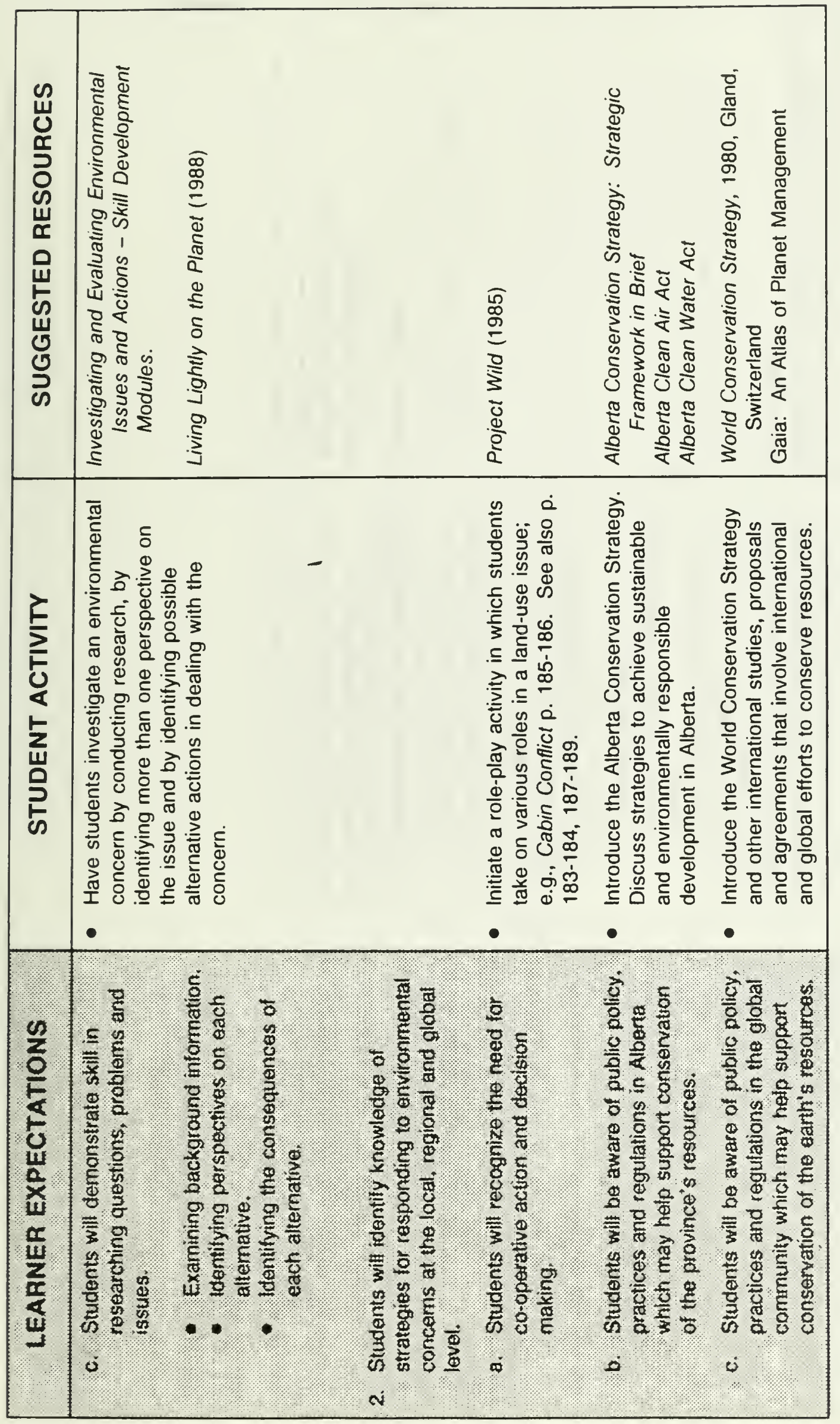




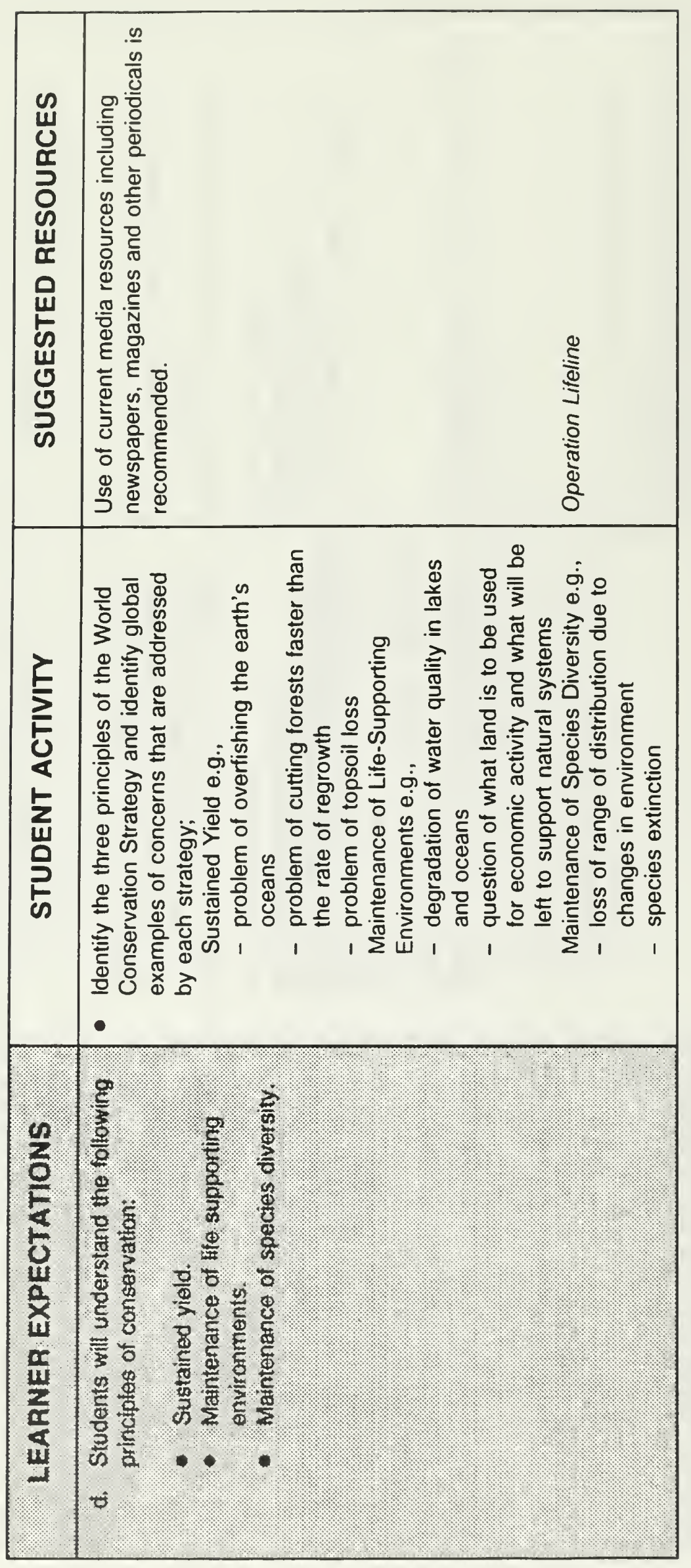




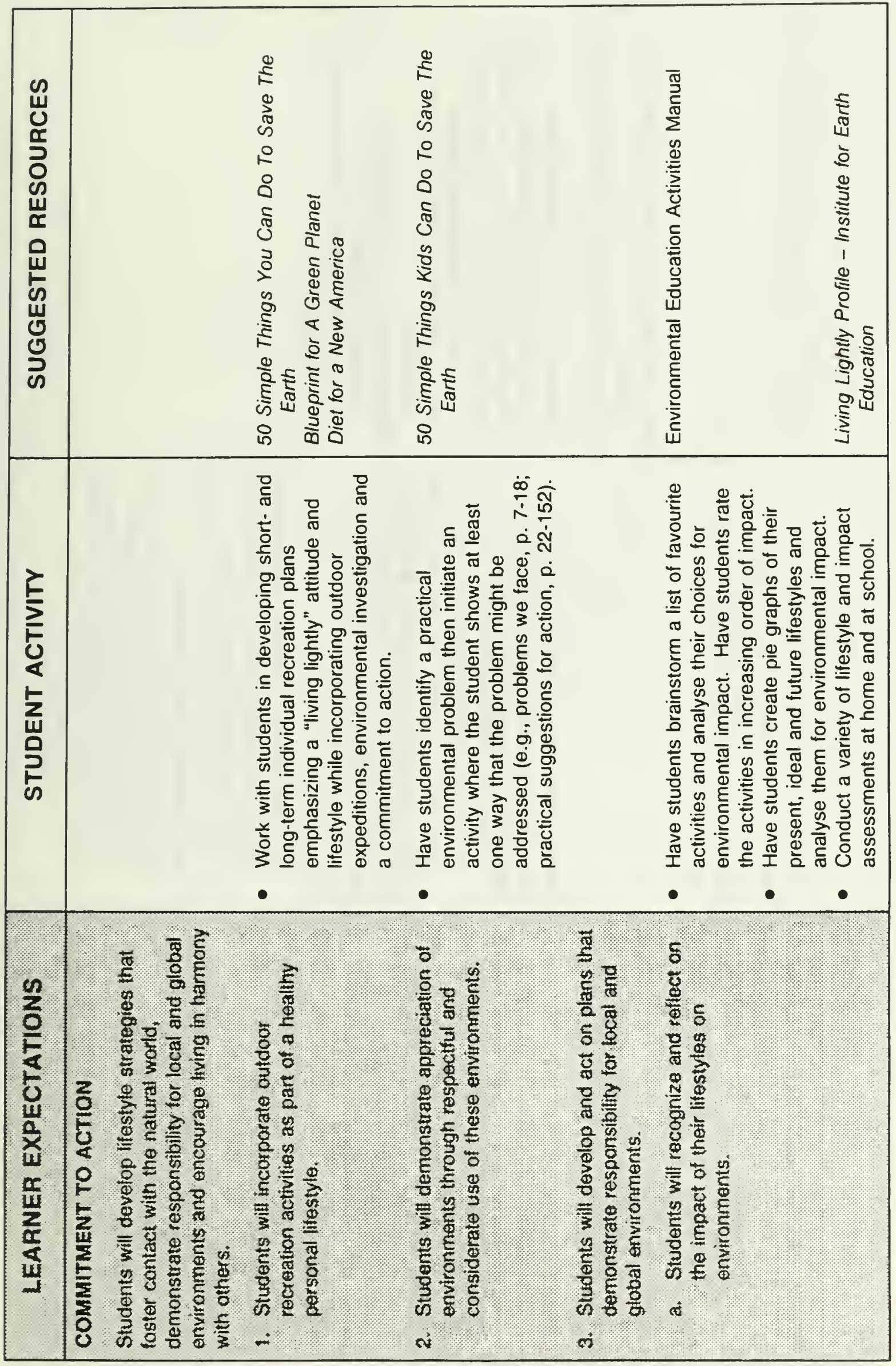




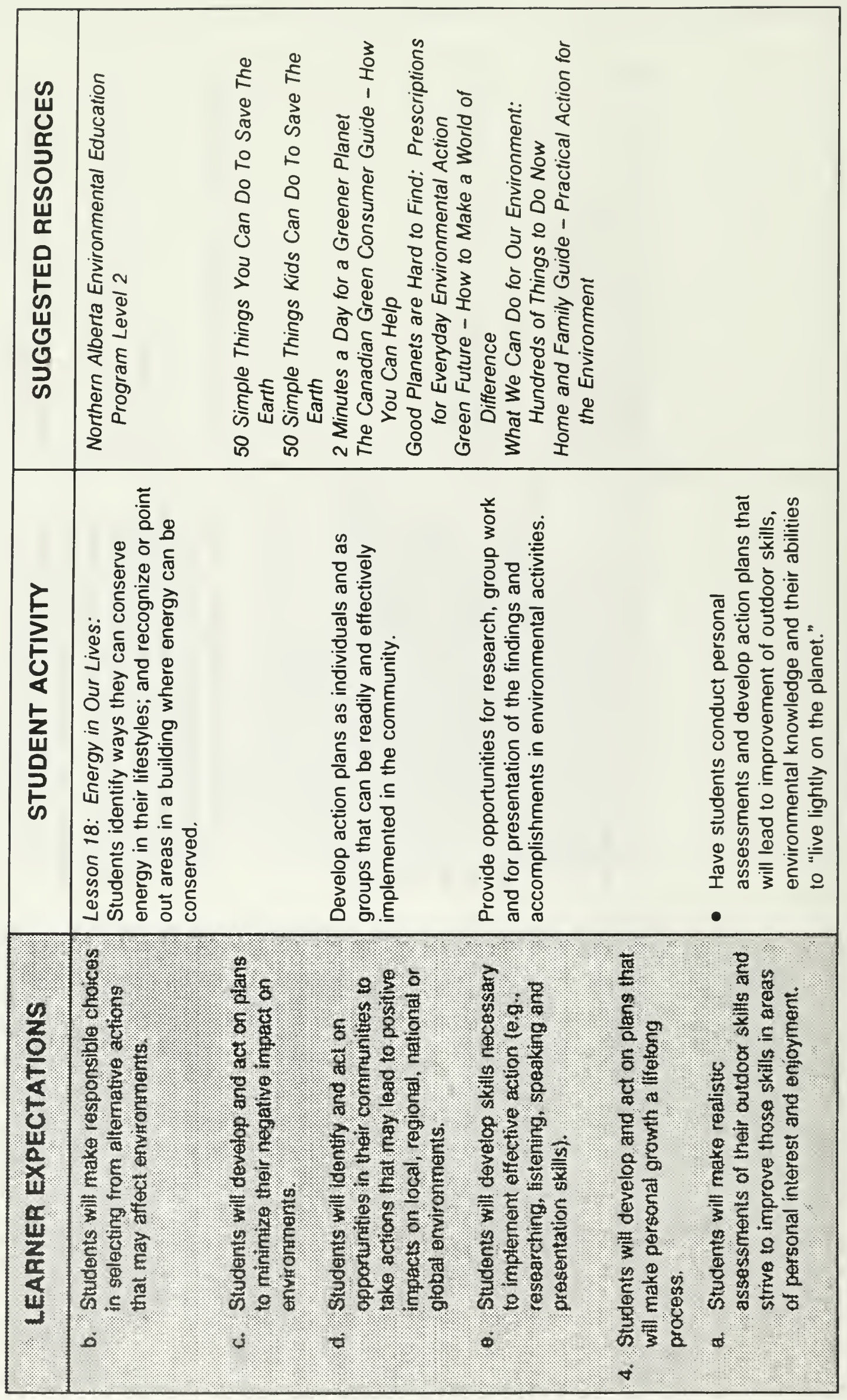




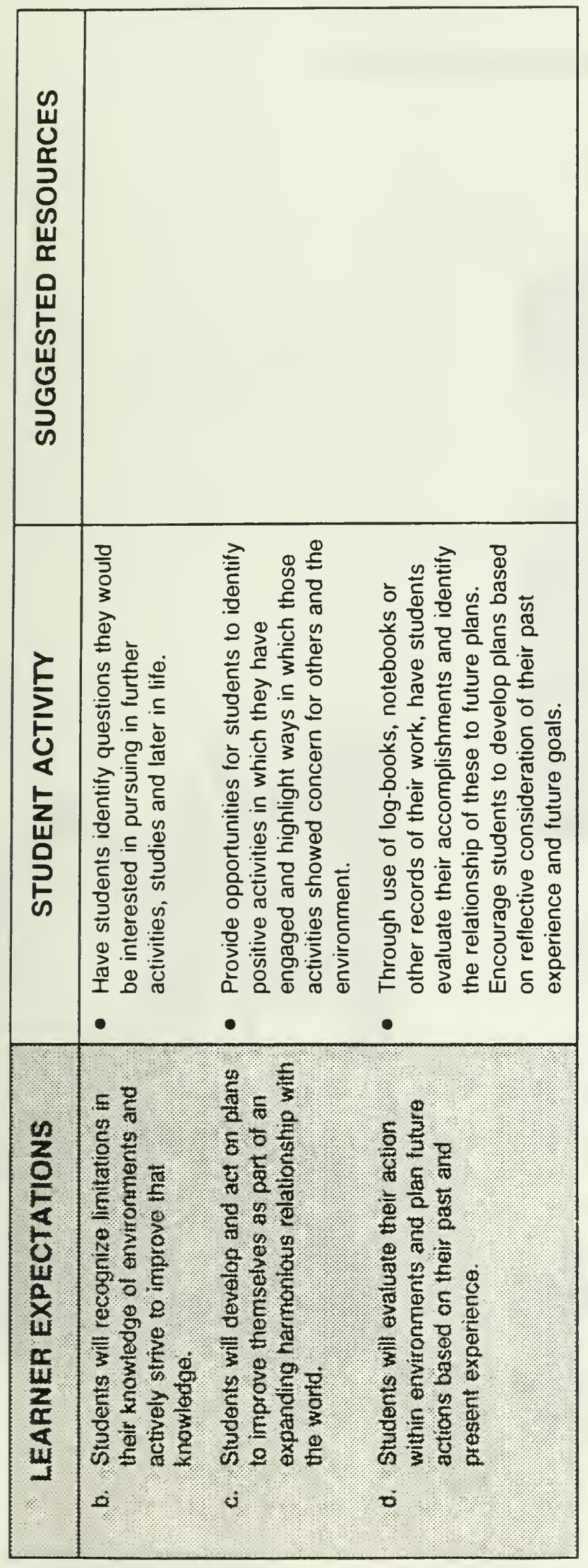




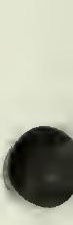



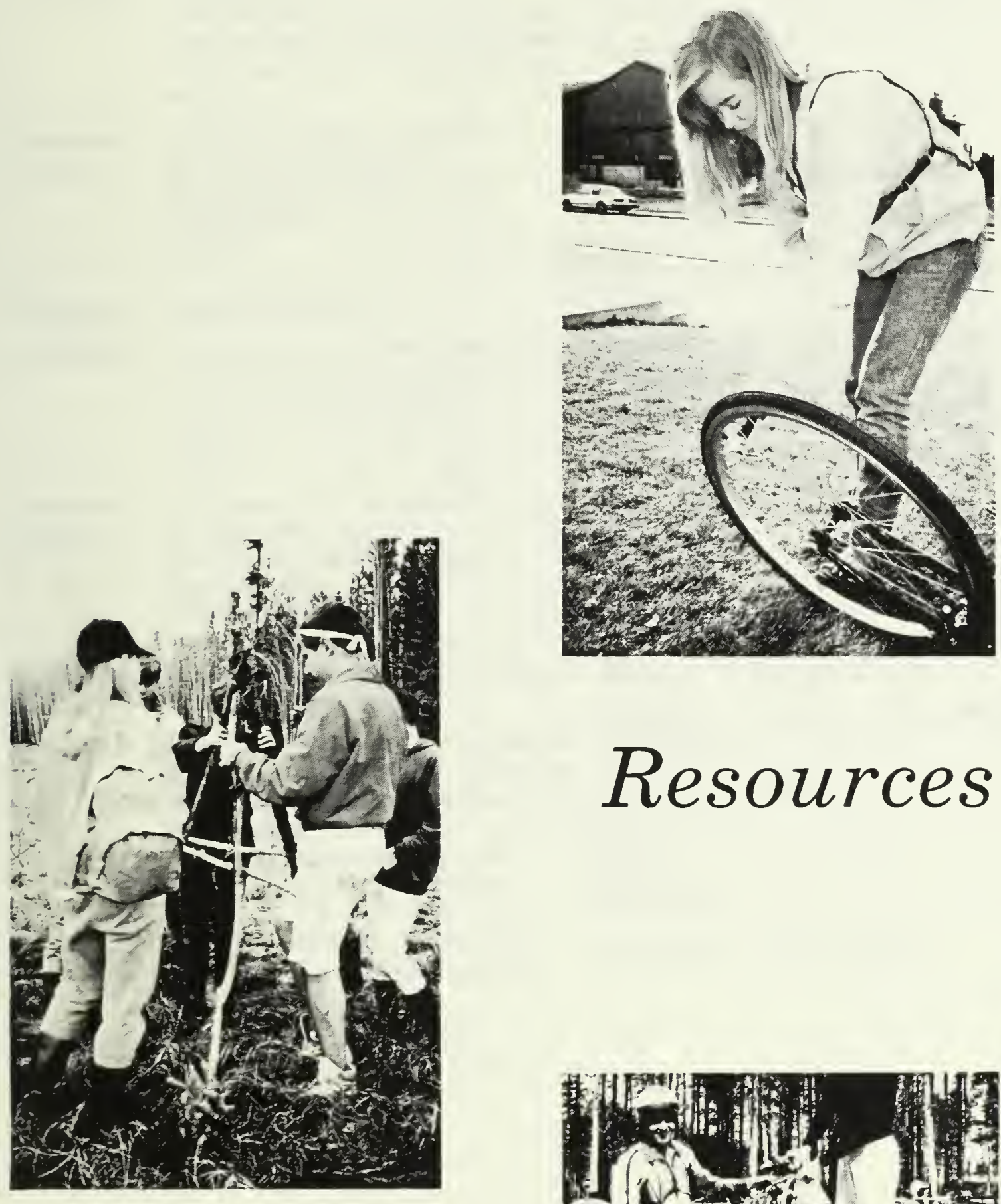

Chapter

\section{Resources}
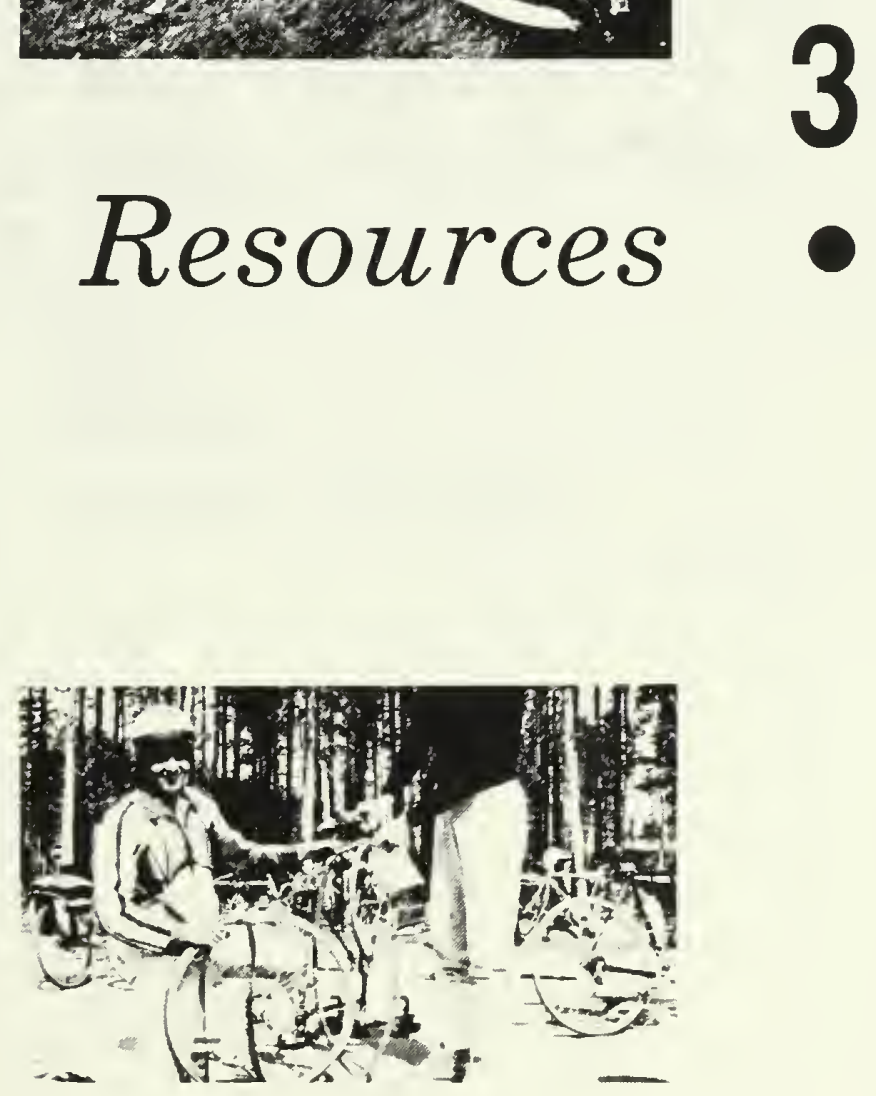
- 
Title/Name Alberta Clean Air Act

Annotation Contains the conclusions and recommendations of the review of the Clean Air Act - a report to the Minister of the Environment. Available free on request.

Distributor Environment Council of Alberta

Address

\section{8th Floor Weber Centre}

5555 Calgary Trail Southbound

Edmonton, $A B$

T6H 5P9
Author

Publisher/ Environment Council of Alberta

Producer

Publication 1985

Date

ISBN

Est. Cost No charge direct

Phone No. (403) 427-5792

\section{Title/Name Alberta Clean Water Act \\ Annotation Conclusions and recommendations of the review of the Clean Water Act - a report to the Minister of the Environment. Available free on request. \\ Distributor Environment Council of Alberta Address 8th Floor Weber Centre 5555 Calgary Trail Southbound Edmonton, $\mathrm{AB}$ T6H 5P9}

\section{Author}

Publisher/ Environment Council of Alberta Producer

Publication 1985

Date

ISBN

\section{Est. Cost}

Phone No. (403) 427-5792
Title/Name Bicycling Level I: Instructor's Manual, Course Handouts

Annotation Designed for beginners and occasional cyclists. Touches on many aspects of cycling from maintenance to touring to effective cycling techniques.

Encourages students to continue cycling education and enjoyment.

Participant Handouts (ED04) 1.95 Instruction (ED03) 7.25 (plus 15\% shipping and handling)

Distributor Canadian Cycling Association Address $\quad 810-1600$ James Naismith Drive Gloucester, ON K1B 5N4

\section{Author Bernhardt, Louis \\ Publisherl Canadian Cycling Association \\ Producer \\ Publication 1986 \\ Date}

ISBN

\section{Est. Cost}

Phone No. (613) 748-5629 


\begin{tabular}{|c|c|c|c|}
\hline \multirow{2}{*}{$\begin{array}{l}\text { Title/Name } \\
\text { Annotation }\end{array}$} & \multirow{3}{*}{$\begin{array}{l}\text { Canadian Green Consumer Guide, } \\
\text { The: How You Can Help } \\
\text { Ways of saving money while saving the } \\
\text { earth. Preface by Margaret Atwood. }\end{array}$} & \multicolumn{2}{|l|}{ Author } \\
\hline & & Publisher/ & McClelland and Stewart \\
\hline & & Publication & 1989 \\
\hline \multirow{4}{*}{$\begin{array}{l}\text { Distributor } \\
\text { Address }\end{array}$} & \multirow{4}{*}{$\begin{array}{l}\text { McClelland and Stewart } \\
25 \text { Hollinger Road } \\
\text { Toronto, ON } \\
\text { M4B 3G2 }\end{array}$} & Date & \\
\hline & & ISBN & 0771071620 \\
\hline & & Est. Cost & $\$ 10.20$ \\
\hline & & Phone No. & $(416) 751-9345$ \\
\hline Title/Name & Canoe Tripping & Author & Kelba, N. et al. \\
\hline Annotation & $\begin{array}{l}\text { One of } 10 \text { recommended support } \\
\text { modules in the "Peer Booklet Series." }\end{array}$ & $\begin{array}{l}\text { Publisher/ } \\
\text { Producer }\end{array}$ & Calgary Board of Education \\
\hline \multirow{6}{*}{$\begin{array}{l}\text { Distributor } \\
\text { Address }\end{array}$} & \multirow{6}{*}{$\begin{array}{l}\text { Calgary Board of Education } \\
\text { Physical Education Department } \\
515 \text { MacLeod Trail S.E. } \\
\text { Calgary, AB } \\
\text { T2G 2L9 }\end{array}$} & Publication & 1983 \\
\hline & & Date & \\
\hline & & ISBN & \\
\hline & & Est. Cost & \\
\hline & & Phone No. & (403) 294-8206 \\
\hline & & Fax No. & (403) 294-8336 \\
\hline
\end{tabular}

\section{Title/Name Communication Skills I: Course Conductor Manual}

Annotation Focuses on the verbal and non-verbal interactions between individuals, paraphrasing, behaviour description, description of feelings and perception checks. Communication Skills I is one course of a group of three known collectively as the Leadership Development Program.

Distributor Address
Blue Lake Centre

Box 6150

Hinton, $A B$

T7V $1 \times 5$
Author Benson, Lyle; Matishak, R.

Publisher/ Alberta Recreation and Parks

Producer

Publication 1985

Date

ISBN

Est. Cost No charge

Phone No. (403) 865-4741

Fax No. (403) 865-5655 


\section{AUTHORIZED RESOURCES}

$\begin{array}{ll}\text { Title/Name } & \text { Conceptual Encounters } \\ \text { Annotation } & \begin{array}{l}\text { Focuses on developing a single } \\ \text { ecological concept, flowing in small } \\ \text { steps from the concrete to the abstract } \\ \text { level of understanding. }\end{array} \\ & \begin{array}{l}\text { Each activity requires } 1 \text { to } 1.5 \text { hours to } \\ \text { complete. Ideal for field trip } \\ \text { experiences or nature centre visits. }\end{array} \\ & \begin{array}{l}\text { Appropriate up to end of Grade } 7 . \\ \text { (Part of the Earth Keeper Program.) }\end{array} \\ \text { Distributor } & \text { Institute for Earth Education } \\ \text { Address } & \text { Publications Service } \\ & \text { P.O. Box } 880 . \text { Station "G" } \\ & \text { Calgary, AB } \\ & \text { T3A } 2 \mathrm{G} 6\end{array}$

Title/Name Conceptual Encounters

Author

Van Matre, Steve et al.

Publisher/

Producer

Publication 1987

Date

ISBN

Est. Cost $\$ 52.15$

Phone No. (403) 246-6611

\section{Title/Name Cranking Out Adventure: A Bike Leader's Guide to Trial and Error Touring}

Annotation A useful guide for teachers planning on leading cycle tours. Provides good ideas on organization, camping and avoiding common pitfalls and hazards.

Distributor

Address
Author

Publisher/

Producer

Publication

Date

ISBN

Est. Cost

Phone No.
Rohnke, Karl

Project Adventure

1977

0934387044

$\$ 5.10$

(508) 468-7981

01936

U.S.A.

TItle/Name Dryland Boating: A Resource Manual for Boating Safety Instruction

Annotation An excellent in-class program dealing with preparation, risk, reduction and survival skills for smallcraft boaters.

Distributor Canadian Red Cross Society

Address
9931-106 Street

Edmonton, $\mathrm{AB}$

T5K 1E2

$\begin{array}{ll}\begin{array}{l}\text { Author } \\ \text { Publisher/ } \\ \text { Producer }\end{array} & \begin{array}{l}\text { Morley, Helen } \\ \text { Canadian Red Cross Society }\end{array} \\ \text { Publication } & 1988 \\ \text { Date } & \\ \text { ISBN } & 092123421 X \\ \text { Est. Cost } & \$ 22.70 \\ \text { Phone No. } & \text { (403) 423-2680 }\end{array}$




\begin{tabular}{|c|c|c|c|}
\hline \multirow[t]{2}{*}{ Title/Name } & \multirow{2}{*}{$\begin{array}{l}\text { Earthkeepers: Four Keys for Helping } \\
\text { Young People Live in Harmony With } \\
\text { the Earth }\end{array}$} & Author & $\begin{array}{l}\text { Van Matre, Steve; Johnson, } \\
\text { Bruce }\end{array}$ \\
\hline & & $\begin{array}{l}\text { Publisher/ } \\
\text { Producer }\end{array}$ & Institute for Earth Education \\
\hline Annotation & $\begin{array}{l}\text { Four keys for helping young people live } \\
\text { in harmony with the earth. }\end{array}$ & Publication & 1987 \\
\hline \multirow[t]{4}{*}{$\begin{array}{l}\text { Distributor } \\
\text { Address }\end{array}$} & \multirow{4}{*}{$\begin{array}{l}\text { Institute for Earth Education } \\
\text { Publications Services } \\
\text { P.O. Box } 880 \text {, Station "G" } \\
\text { Calgary, AB } \\
\text { T3A 2G6 }\end{array}$} & Date & \\
\hline & & ISBN & 0917011015 \\
\hline & & & $\$ 14.05$ \\
\hline & & Phone No. & $(403) 246-6611$ \\
\hline \multirow[t]{2}{*}{ Title/Name } & \multirow{2}{*}{$\begin{array}{l}\text { Earthwalks: Acclimatization Walks for } \\
\text { a Sensory Encounter with the Natural } \\
\text { World (Earthwalks: Earth Magic and } \\
\text { Snow Walks) }\end{array}$} & Author & Hoesse, K. \\
\hline & & $\begin{array}{l}\text { Publisher/ } \\
\text { Producer }\end{array}$ & Institute for Earth Education \\
\hline \multirow[t]{3}{*}{ Annotation } & \multirow{3}{*}{$\begin{array}{l}\text { A collection of natural awareness } \\
\text { activities that can be used as the } \\
\text { components of a nature awareness } \\
\text { walk. }\end{array}$} & Publication & 1980 \\
\hline & & Date & \\
\hline & & ISBN & \\
\hline \multirow{2}{*}{$\begin{array}{l}\text { Distributor } \\
\text { Address }\end{array}$} & \multirow{2}{*}{$\begin{array}{l}\text { Institute for Earth Education } \\
\text { Publications Services } \\
\text { P.O. Box } 880 \text {, Station "G" } \\
\text { Calgary, AB } \\
\text { T3A 2G6 }\end{array}$} & Est. Cost & $\$ 24.45$ \\
\hline & & Phone No. & (403) 246-6611 \\
\hline \multirow[t]{2}{*}{ Title/Name } & \multirow{2}{*}{$\begin{array}{l}\text { Ecology Studies Of Lakes in Alberta: } \\
\text { Observation Notes }\end{array}$} & Author & Stoker, D. \\
\hline & & Publisher/ & Alberta Environment \\
\hline \multirow[t]{4}{*}{ Annotation } & \multirow{4}{*}{$\begin{array}{l}\text { Deals with the ecology of freshwater } \\
\text { environments. Unit introduces human } \\
\text { impact on lake environments and } \\
\text { involves students in the methods and } \\
\text { technology employed to study lakes. } \\
\text { Workshops provided. }\end{array}$} & Producer & \\
\hline & & Publication & 1988 \\
\hline & & Date & \\
\hline & & ISBN & \\
\hline \multirow{2}{*}{$\begin{array}{l}\text { Distributor } \\
\text { Address }\end{array}$} & Alberta Environment & Est. Cost & Not available yet \\
\hline & $\begin{array}{l}\text { Oxbridge Place, 12th Floor } \\
\text { 9820-106 Street } \\
\text { Edmonton, AB } \\
\text { T5K 2J6 }\end{array}$ & Phone No. & (403) 427-6310 \\
\hline
\end{tabular}




\section{AUTHORIZED RESOURCES}

Title/Name Ecology Studies Of Lakes In Alberta: Student Book

Annotation Deals with the ecology of freshwater environments. Unit introduces human impact on lake environments and involves students in the methods and technology employed to study lakes. Workshops provided.

\section{Distributor Alberta Environment}

Address

\section{Oxbridge Place, 12th Floor}

9820-106 Street

Edmonton, $\mathrm{AB}$

T5K 2J6
Author

Publisher/

Producer

Publication 1988

Date

ISBN

Est. Cost Not available yet

Phone No. (403) 427-6310

Stoker, D.
Alberta Environment

Ecology Studies Of Lakes In Alberta: Student Worksheets

Annotation Deals with the ecology of freshwater environments. Unit introduces human impact on lake environments and involves students in the methods and technology employed to study lakes. Workshops provided.

Distributor Alberta Environment Address Oxbridge Place, 12th Floor 9820-106 Street

Author Stoker, D.

Publisher/ Alberta Environment Producer

Publication 1988

Date

ISBN

Est. Cost Not available yet

Phone No. (403) 427-6310

Edmonton, $A B$ T5K 2J6

Title/Name Ecology Studies Of Lakes in Alberta: Teacher's Guide

Annotation Deals with the ecology of freshwater environments. Unit introduces human impact on lake environments and involves students in the methods and technology employed to study lakes. Workshops provided.

Distributor Environmental Education Branch Address Water Literacy Program 9820-106 Street Edmonton, $\mathrm{AB}$ T5K 2J6
Author Stoker, D.

Publisher/ Producer

Publication 1989

Date

ISBN

Est. Cost Free with workshop

Phone No. (403) 427-6310 


\begin{tabular}{|c|c|c|c|}
\hline Title/Name & Energy News Kit, The & Author & Alberta Energy \\
\hline \multirow[t]{4}{*}{ Annotation } & \multirow{4}{*}{$\begin{array}{l}\text { This kit is designed to teach concepts } \\
\text { of renewable energy and make } \\
\text { students aware of our dependence on } \\
\text { non-renewable energy sources. It } \\
\text { challenges students to commit to } \\
\text { energy-saving actions. }\end{array}$} & $\begin{array}{l}\text { Publisher/ } \\
\text { Producer }\end{array}$ & Alberta Energy \\
\hline & & Publication & 1989 \\
\hline & & Date & \\
\hline & & ISBN & 0864995687 \\
\hline \multirow{2}{*}{$\begin{array}{l}\text { Distributor } \\
\text { Address }\end{array}$} & \multirow{2}{*}{$\begin{array}{l}\text { Alberta Energy } \\
\text { Energy Efficiency Branch } \\
\text { 2nd Floor, Highfield Place } \\
10010-106 \text { Street } \\
\text { Edmonton, AB } \\
\text { T5J 3L8 }\end{array}$} & Est. Cost & No charge \\
\hline & & Phone No. & (403) 427-5200 \\
\hline \multirow[t]{2}{*}{ Title/Name } & \multirow{2}{*}{$\begin{array}{l}\text { Fifty Simple Things Kids Can Do To } \\
\text { Save The Earth (1st ed.) }\end{array}$} & Author & Javna, John \\
\hline & & Publisher/ & Andrews \& McMeel \\
\hline \multirow[t]{3}{*}{ Annotation } & \multirow{3}{*}{$\begin{array}{l}\text { A summary of the environmental } \\
\text { problems we face and practical things } \\
\text { that young people can do to make a } \\
\text { difference. }\end{array}$} & Producer & \\
\hline & & Publication & 1990 \\
\hline & & Date & \\
\hline \multirow{3}{*}{$\begin{array}{l}\text { Distributor } \\
\text { Address }\end{array}$} & \multirow{3}{*}{$\begin{array}{l}\text { Gage Educational Publishing } \\
164 \text { Commander Boulevard } \\
\text { Agincourt, ON } \\
\text { M1S } 3 C 7\end{array}$} & ISBN & 0836223012 \\
\hline & & Est. Cost & $\$ 4.25$ \\
\hline & & Phone No. & (416) 293-8141 \\
\hline Title/Name & $\begin{array}{l}\text { Fifty Simple Things You Can Do To } \\
\text { Save The Earth }\end{array}$ & Author & Earthworks Group \\
\hline \multirow[t]{2}{*}{ Annotation } & \multirow{2}{*}{$\begin{array}{l}\text { A summary of the environmental } \\
\text { problems we face and practical things } \\
\text { we can do to make a difference. }\end{array}$} & $\begin{array}{l}\text { Publisher/ } \\
\text { Producer }\end{array}$ & Earthworks Press \\
\hline & & Publication & 1989 \\
\hline \multirow{4}{*}{$\begin{array}{l}\text { Distributor } \\
\text { Address }\end{array}$} & \multirow{4}{*}{$\begin{array}{l}\text { Earthworks Press } \\
\text { Box } 25 \\
1400 \text { Shatuck Avenue } \\
\text { Berkley, CA } \\
94709 \\
\text { U.S.A. }\end{array}$} & Date & \\
\hline & & ISBN & 0929634063 \\
\hline & & Est. Cost & \\
\hline & & Phone No. & (415) 841-5866 \\
\hline
\end{tabular}




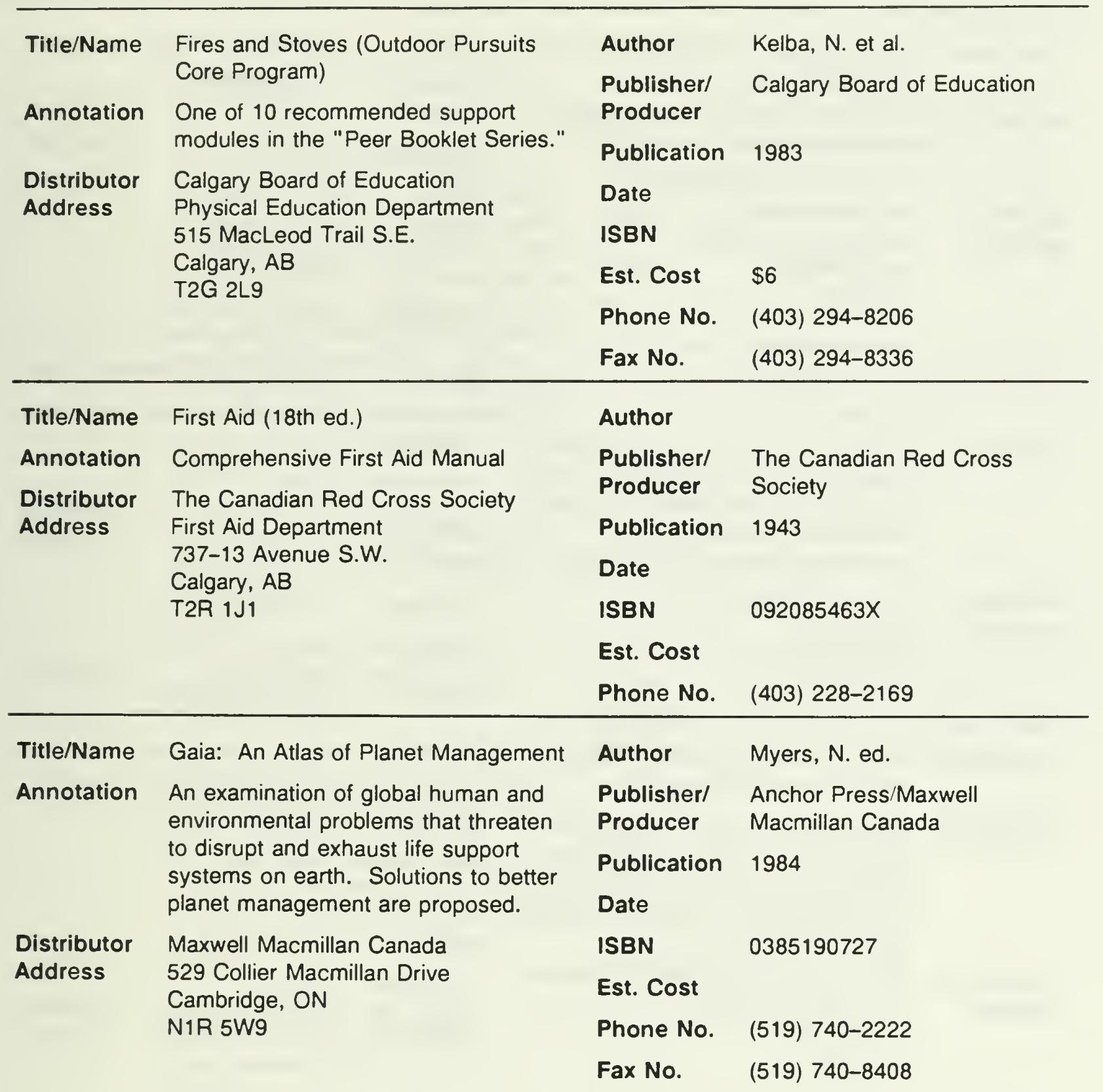




\begin{tabular}{|c|c|c|c|}
\hline \multirow[t]{2}{*}{ Title/Name } & \multirow{2}{*}{$\begin{array}{l}\text { Green Future: How to Make a World } \\
\text { of Difference }\end{array}$} & Author & Johnson, Lorraine \\
\hline & & Publisher/ & Penguin Books \\
\hline \multirow[t]{2}{*}{ Annotation } & \multirow{2}{*}{$\begin{array}{l}\text { Explores major environmental issues } \\
\text { and offers practical suggestions for } \\
\text { daily positive action. }\end{array}$} & Producer & \\
\hline & & Publication & 1990 \\
\hline \multirow{4}{*}{$\begin{array}{l}\text { Distributor } \\
\text { Address }\end{array}$} & \multirow{4}{*}{$\begin{array}{l}\text { Penguin Books } \\
1220 \text { Nicholson Road } \\
\text { Newmarket, ON } \\
\text { L3V } 7 \text { V2 }\end{array}$} & Date & \\
\hline & & ISBN & 0140123016 \\
\hline & & Est. Cost & $\$ 10.40$ \\
\hline & & Phone No. & $(416) 836-6730$ \\
\hline Title/Name & $\begin{array}{l}\text { Group Relations I: Course Conductor } \\
\text { Manual }\end{array}$ & Author & Benson, Lyle; Matishak, Rick \\
\hline \multirow[t]{4}{*}{ Annotation } & \multirow{4}{*}{$\begin{array}{l}\text { Examines how a group develops by } \\
\text { focusing on group interactions. Group } \\
\text { Relations I is one course of a group of } \\
\text { three known collectively as the } \\
\text { Leadership Development Program. }\end{array}$} & $\begin{array}{l}\text { Publisher/ } \\
\text { Producer }\end{array}$ & Alberta Recreation and Parks \\
\hline & & Publication & 1985 \\
\hline & & Date & \\
\hline & & ISBN & \\
\hline \multirow[t]{3}{*}{$\begin{array}{l}\text { Distributor } \\
\text { Address }\end{array}$} & \multirow{3}{*}{$\begin{array}{l}\text { Blue Lake Centre } \\
\text { Box } 6150 \\
\text { Hinton, AB } \\
\text { T7V } 1 \times 5\end{array}$} & Est. Cost & No charge \\
\hline & & Phone No. & (403) 865-4741 \\
\hline & & Fax No. & (403) 865-5655 \\
\hline Title/Name & $\begin{array}{l}\text { Home and Family Guide: Practical } \\
\text { Action for the Environment }\end{array}$ & Author & Ward-Whate, Louis \\
\hline \multirow[t]{3}{*}{ Annotation } & \multirow{3}{*}{$\begin{array}{l}\text { Clear, practical information on how to } \\
\text { tackle environmental issues in day-to- } \\
\text { day life, recognizing that collectively we } \\
\text { can have a tremendous influence on } \\
\text { the environment. }\end{array}$} & $\begin{array}{l}\text { Publisher/ } \\
\text { Producer }\end{array}$ & $\begin{array}{l}\text { The Harmony Foundation of } \\
\text { Canada }\end{array}$ \\
\hline & & $\begin{array}{l}\text { Publication } \\
\text { Date }\end{array}$ & 1989 \\
\hline & & ISBN & 0929010019 \\
\hline $\begin{array}{l}\text { Distributor } \\
\text { Address }\end{array}$ & $\begin{array}{l}\text { The Harmony Foundation of Canada } \\
\text { P.O. Box } 3444 \text {, Station "D" } \\
\text { Ottawa, ON } \\
\text { K1P 6P9 }\end{array}$ & $\begin{array}{l}\text { Est. Cost } \\
\text { Phone No. }\end{array}$ & $\begin{array}{l}\$ 5.95 \\
(613) 230-7353\end{array}$ \\
\hline
\end{tabular}




\section{AUTHORIZED RESOURCES}

Title/Name Kananaskis Country Environmental

Education Teaching Activity Guide:

Earth Science

Annotation This guidebook has three units: Earth History, Geologic Processes and Geologic Materials. Activities in each unit have been arranged by topics that elaborate on one or more key concepts of environmental education. These key concepts include: change, adaptation, ecosystems, interdependence, cycles, resources and technology. While building on the students' knowledge and skills, the activities also encourage an examination of personal values and attitudes.

Distributor Calgary Board of Education

Address Environmental and Outdoor Education 5th Floor, 515 MacLeod Trail S.E.

Calgary, $A B$

T2G 2 L9
Title/Name Leadership Assessment I: Course Conductor Manual

Annotation A series of well-planned activities that are divided into three types of skills: program skills, people skills and selfskills. The aim of this program is to train leaders to be more effective. Leadership Assessment I is one course of a group of three known collectively as the Leadership Development Program.

Distributor Alberta Recreation and Parks Address 7 th Floor, Standard Life Centre 10405 Jasper Avenue

Edmonton, $\mathrm{AB}$

T5J 3N4
Author

Publisher/

Producer

Publication 1987

Date

ISBN

Est. Cost Not determined

Phone No. (403) 294-8205
Lengsfeld, R. et al.

Kananaskis Country, Calgary

Board of Education, Alberta

Environment Research Trust

0140123016
Author

Publisher/

Producer

Publication 1985

Date

ISBN

Est. Cost No charge

Phone No. (403) 427-8758 


\begin{tabular}{|c|c|c|c|}
\hline \multirow[t]{2}{*}{ Title/Name } & \multirow{2}{*}{$\begin{array}{l}\text { Menu Planning and Food Preparation } \\
\text { (Outdoor Pursuits Core Program) }\end{array}$} & Author & Kelba, N. et al. \\
\hline & & Publisher/ & Calgary Board of Education \\
\hline \multirow[t]{2}{*}{ Annotation } & \multirow{2}{*}{$\begin{array}{l}\text { One of } 10 \text { recommended support } \\
\text { modules in the "Peer Booklet Series." }\end{array}$} & Producer & \\
\hline & & Publication & 1983 \\
\hline \multirow{5}{*}{$\begin{array}{l}\text { Distributor } \\
\text { Address }\end{array}$} & \multirow{5}{*}{$\begin{array}{l}\text { Calgary Board of Education } \\
\text { Physical Education Department } \\
515 \text { MacLeod Trail S.E. } \\
\text { Calgary, AB } \\
\text { T2G 2L9 }\end{array}$} & Date & \\
\hline & & ISBN & \\
\hline & & Est. Cost & $\$ 6$ \\
\hline & & Phone No. & (403) 294-8206 \\
\hline & & Fax No. & (403) 294-8336 \\
\hline \multirow[t]{2}{*}{ Title/Name } & \multirow{2}{*}{$\begin{array}{l}\text { Navigation (Outdoor Pursuits Core } \\
\text { Program) }\end{array}$} & Author & Kelba, N. et al. \\
\hline & & Publisher/ & Calgary Board of Education \\
\hline \multirow{2}{*}{ Annotation } & \multirow{2}{*}{$\begin{array}{l}\text { One of } 10 \text { recommended support } \\
\text { modules in the "Peer Booklet Series." }\end{array}$} & Producer & \\
\hline & & Publication & 1983 \\
\hline \multirow[t]{5}{*}{$\begin{array}{l}\text { Distributor } \\
\text { Address }\end{array}$} & \multirow{5}{*}{$\begin{array}{l}\text { Calgary Board of Education } \\
\text { Physical Education Department } \\
515 \text { MacLeod Trail S.E. } \\
\text { Calgary, AB } \\
\text { T2G 2L9 }\end{array}$} & Date & \\
\hline & & ISBN & \\
\hline & & Est. Cost & $\$ 9.10$ \\
\hline & & Phone No. & (403) 294-8206 \\
\hline & & Fax No. & (403) 294-8336 \\
\hline Title/Name & Northern Bushcraft (expanded ed.) & Author & Kochanski, Mors \\
\hline \multirow[t]{6}{*}{ Annotation } & \multirow{6}{*}{$\begin{array}{l}\text { This book provides practical } \\
\text { information for everyday living in the } \\
\text { northern forest. More than a manual, it } \\
\text { discusses the basic skills that allow } \\
\text { survival in challenging situations. } \\
\text { Topics such as firecraft, axecraft, } \\
\text { knifecraft, sawcraft, rope work, shelter } \\
\text { and environment opportunities are } \\
\text { discussed. }\end{array}$} & $\begin{array}{l}\text { Publisher/ } \\
\text { Producer }\end{array}$ & Lone Pine Publishing \\
\hline & & Publication & 1988 \\
\hline & & Date & \\
\hline & & ISBN & 0919433510 \\
\hline & & Est. Cost & $\$ 10.30$ \\
\hline & & Phone No. & (403) 433-9333 \\
\hline $\begin{array}{l}\text { Distributor } \\
\text { Address }\end{array}$ & $\begin{array}{l}\text { Lone Pine Publishing } \\
\text { Suite 206, 10426-81 Avenue } \\
\text { Edmonton, AB } \\
\text { T6E 1X5 }\end{array}$ & & \\
\hline
\end{tabular}




\section{AUTHORIZED RESOURCES}

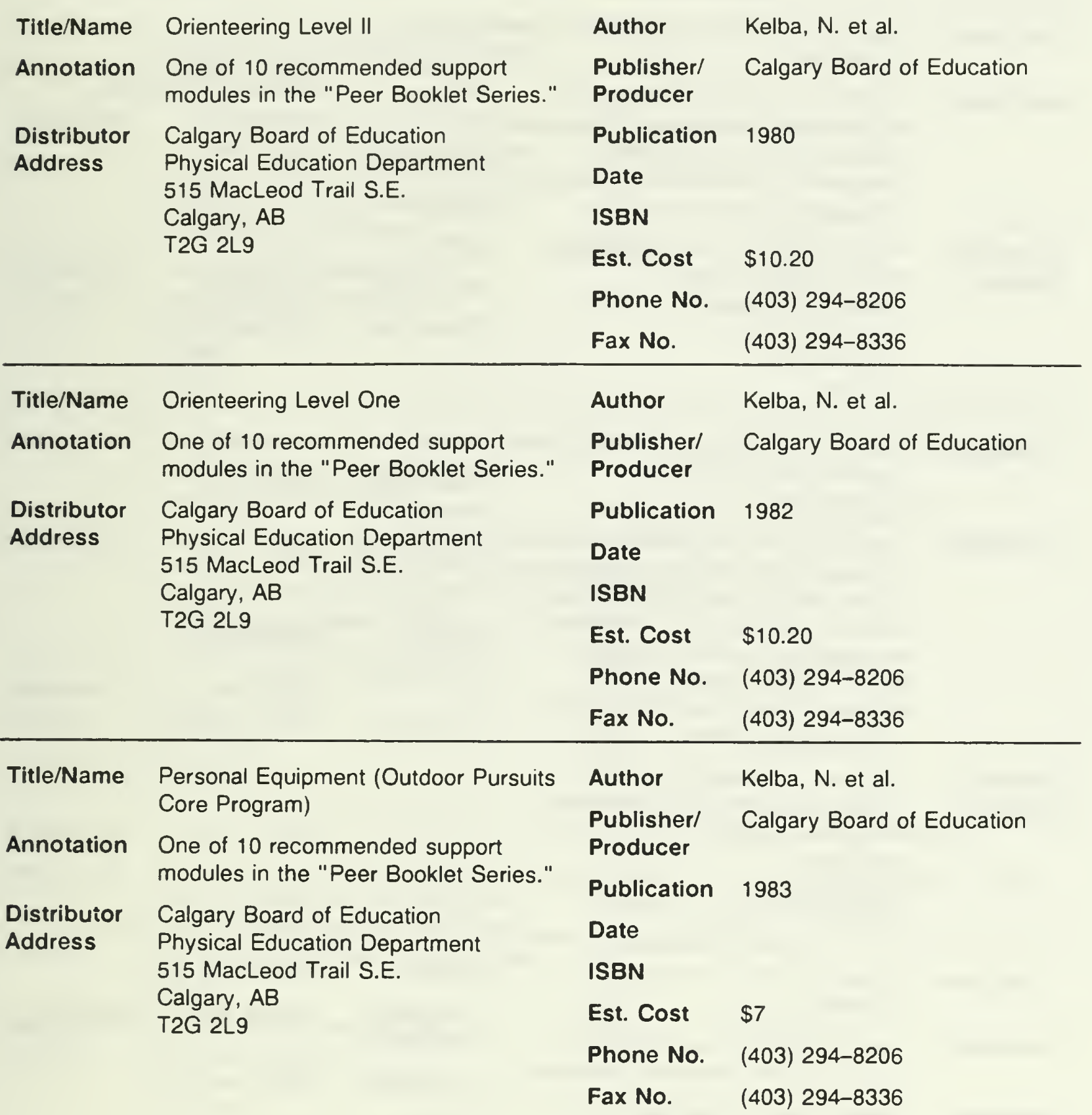




$\begin{array}{ll}\text { Title/Name } & \begin{array}{l}\text { Project Wild: Secondary Activity Guide } \\ \text { (revised ed.) }\end{array} \\ \text { Annotation } & \begin{array}{l}\text { A collection of interdisciplinary and } \\ \text { supplementary environmental education } \\ \text { materials emphasizing wildlife } \\ \text { conservation. Activities are indexed by } \\ \text { topic, grade, subject and skill. }\end{array} \\ \text { Distributor } & \text { Alberta Forestry, Lands and Wildlife } \\ \text { Address } & \begin{array}{l}\text { Fish and Wildlife Division } \\ \text { Conservation Education }\end{array} \\ & \begin{array}{l}\text { 14515-122 Avenue } \\ \text { Edmonton, AB } \\ \text { T5L } 2 W 4\end{array}\end{array}$

Author

Publisher/ Western Regional

Producer Environmental Education Council

Publication 1985

Date

ISBN

Est. Cost No charge

Phone No. (403) 427-3574

\begin{tabular}{|c|c|}
\hline Title/Name & $\begin{array}{l}\text { Safety Oriented Guidelines for Ou } \\
\text { Education Leadership and } \\
\text { Programming }\end{array}$ \\
\hline Annotation & $\begin{array}{l}\text { Leadership and programming stan } \\
\text { for expeditions and backcountry tr } \\
\text { Topics such as qualified leadershi } \\
\text { navigation programming standards } \\
\text { some activity-specific guidelines, } \\
\text { specific activity checklist and } \\
\text { equipment lists as well as post-trip } \\
\text { activities and evaluation. }\end{array}$ \\
\hline $\begin{array}{l}\text { Distributor } \\
\text { Address }\end{array}$ & $\begin{array}{l}\text { Canadian Association of Health, } \\
\text { Physical Education and Recreatic } \\
1600 \text { James Naismith Drive } \\
\text { Suite } 606 \\
\text { Gloucester, ON } \\
\text { K1B 5N4 }\end{array}$ \\
\hline
\end{tabular}

\section{Author Hanna, Glenda \\ Publisher/ CAHPER Producer}

Publication 1986

Date

ISBN

0919068529

Est. Cost $\$ 13.50$

Phone No. (613) 748-5622

$\begin{array}{ll}\text { Title/Name } & \begin{array}{l}\text { Shelters (Outdoor Pursuits Core } \\ \text { Program) }\end{array} \\ \text { Annotation } & \begin{array}{l}\text { One of } 10 \text { recommended support } \\ \text { modules in the "Peer Booklet Series." }\end{array} \\ \text { Distributor } & \begin{array}{l}\text { Calgary Board of Education } \\ \text { Address }\end{array} \\ & \begin{array}{l}\text { Physical Education Department } \\ 515 \text { MacLeod Trail S.E. } \\ \text { Calgary, AB }\end{array} \\ & \text { T2G } 2 \text { L } 9\end{array}$

Author Kelba, N. et al.

Publisher/ Calgary Board of Education

Producer

Publication 1983

Date

ISBN

Est. Cost $\$ 6$

Phone No. (403) 294-8206

Fax No. (403) 294-8336 


\section{AUTHORIZED RESOURCES}

Title/Name Silver Bullets: A Guide To Initiative

Problems, Adventure Games, Stunts and Trust Activities

Annotation A text containing a series of effective activities to bring people together to build trust and to break down the artificial barriers between individuals and groups of individuals.

Distributor Kendall/Hunt Publishing Co.

Address 2460 Kerper Boulevard

Dubuque, IA

52001

U.S.A.
Author Rohnke, K.

Publisher/ Project Adventure

Producer

Publication 1984

Date

ISBN 084035682X

Est. Cost $\$ 24.15$

Phone No. (319) 588-1451
Title/Name Ski Trails in the Canadian Rockies: A Guide to Banff, Jasper, Kootenay and Yoho Parks (revised ed.)

Annotation A complete guide to cross-country skiing in the Rockies. A good introduction to equipment and survival techniques and trails in Banff, Kootenays, Yoho and Jasper parks.

Distributor Summerthought Ltd.

Address P.O. Box 1420

Banff, AB

TOL OCO
Author

Publisher/

Producer

Publication 1981

Date

ISBN

Est. Cost $\$ 6.95$

Phone No. (403) 762-3919 Dave

0919934064
Kunelius, Rick; Biederman,

Summerthought Ltd.

Author

Osgood, W.; Hurley, L.

Title/Name Snowshoe Book, The (3rd ed.)

Publisher/

The Stephen Greene Press/

Producer

Penguin Books

Publication 1983

and tips for the novice as well as the expert.

Date

ISBN

082904324

Est. Cost $\$ 10.20$

Phone No. (416) 475-1571 


\section{AUTHORIZED RESOURCES}

\begin{tabular}{|c|c|c|c|}
\hline \multirow{3}{*}{$\begin{array}{l}\text { Title/Name } \\
\text { Annotation }\end{array}$} & \multirow{3}{*}{$\begin{array}{l}\text { Sunship Earth: An Acclimatization } \\
\text { Program for Outdoor Learning } \\
\text { Includes short (20-minute) concept } \\
\text { path activities to acclimatize students } \\
\text { for outdoor learning. Designed for } \\
\text { students grades } 6 \text { to } 8 \text {. }\end{array}$} & Author & Van Matre, Steve \\
\hline & & $\begin{array}{l}\text { Publisher/ } \\
\text { Producer }\end{array}$ & $\begin{array}{l}\text { American Camping Association/ } \\
\text { Institute for Earth Education }\end{array}$ \\
\hline & & $\begin{array}{l}\text { Publication } \\
\text { Date }\end{array}$ & 1979 \\
\hline \multirow{2}{*}{$\begin{array}{l}\text { Distributor } \\
\text { Address }\end{array}$} & \multirow{2}{*}{$\begin{array}{l}\text { Institute for Earth Education } \\
\text { P.O. Box } 880 \text {, Station "G" } \\
\text { Calgary, AB } \\
\text { T3A 2G6 }\end{array}$} & ISBN & 0876030460 \\
\hline & & $\begin{array}{l}\text { Est. Cost } \\
\text { Phone No. }\end{array}$ & $\begin{array}{l}\$ 18.10 \\
(403) 246-6611\end{array}$ \\
\hline \multirow[t]{2}{*}{ Title/Name } & \multirow{2}{*}{$\begin{array}{l}\text { Trip Planning (Outdoor Pursuits Core } \\
\text { Program) }\end{array}$} & Author & Kelba, N. et al. \\
\hline & & $\begin{array}{l}\text { Publisher/ } \\
\text { Producer }\end{array}$ & Calgary Board of Education \\
\hline Annotation & $\begin{array}{l}\text { One of } 10 \text { recommended support } \\
\text { modules in the "Peer Booklet Series." }\end{array}$ & Publication & 1984 \\
\hline \multirow[t]{5}{*}{$\begin{array}{l}\text { Distributor } \\
\text { Address }\end{array}$} & \multirow{5}{*}{$\begin{array}{l}\text { Calgary Board of Education } \\
\text { Physical Education Department } \\
515 \text { MacLeod Trail S.E. } \\
\text { Calgary, AB } \\
\text { T2G 2L9 }\end{array}$} & Date & \\
\hline & & ISBN & \\
\hline & & Est. Cost & $\$ 9.10$ \\
\hline & & Phone No. & (403) 294-8206 \\
\hline & & Fax No. & (403) 294-8336 \\
\hline Title/Name & \multirow{3}{*}{$\begin{array}{l}\text { Two Minutes a Day for a Greener } \\
\text { Planet } \\
\text { Quick and simple things you can do to } \\
\text { save the earth. }\end{array}$} & Author & Lamb, Marjorie \\
\hline \multirow[t]{2}{*}{ Annotation } & & $\begin{array}{l}\text { Publisher/ } \\
\text { Producer }\end{array}$ & Harper Collins Publishers \\
\hline & & Publication & 1990 \\
\hline \multirow[t]{5}{*}{$\begin{array}{l}\text { Distributor } \\
\text { Address }\end{array}$} & \multirow{5}{*}{$\begin{array}{l}\text { Harper Collins Publishers } \\
1995 \text { Markham Road } \\
\text { Scarborough, ON } \\
\text { M1B 5M8 }\end{array}$} & Date & \\
\hline & & ISBN & 0002156822 \\
\hline & & Est. Cost & $\$ 10.20$ \\
\hline & & Phone No. & (416) $321-2241$ \\
\hline & & & (416) 321-3033 \\
\hline
\end{tabular}




\section{AUTHORIZED RESOURCES}

Title/Name What We Can Do For Our

Environment: Hundreds of Things To

Do Now

Annotation Hundreds of ideas for things that each of us can do to protect and improve our environment.

Distributor Environment Canada,

Address Communications Branch

Room 210

4999-98 Avenue

Edmonton, $A B$

T6B $2 \times 3$
Author Environment Canada

Publisher/ Environment Canada

Producer

Publication 1990

Date

ISBN $\quad 0662175352$

Est. Cost No charge

Phone No. (403) 468-8075 


\begin{tabular}{|c|c|}
\hline Title/Name & $\begin{array}{l}\text { Alberta Fish and Wildlife Conservatio } \\
\text { Education Series: Equipment }\end{array}$ \\
\hline Annotation & $\begin{array}{l}\text { Well-illustrated manual available free } \\
\text { for certified instructors. Ask about } \\
\text { resources and instructor program. } \\
\text { Teachers must be made aware that } \\
\text { illustrations in this book depict male } \\
\text { Caucasians only. The omissions of } \\
\text { illustrations involving females and rad } \\
\text { minorities may be offensive to some } \\
\text { groups; teacher discretion advised. } \\
\text { request will be made to the publisher } \\
\text { incorporate the suggestions in the ne } \\
\text { printing. }\end{array}$ \\
\hline $\begin{array}{l}\text { Distributor } \\
\text { Address }\end{array}$ & $\begin{array}{l}\text { Alberta Forestry, Lands and Wildlife } \\
\text { Fish and Wildlife Division } \\
\text { Conservation Education } \\
\text { 14515-122 Avenue } \\
\text { Edmonton, AB } \\
\text { T5L 2W4 }\end{array}$ \\
\hline
\end{tabular}

Author

Publlsher/ Alberta Forestry, Lands and Producer Wildlife

Publicatlon

Date

ISBN

Est. Cost No charge

Phone No. (403) 427-3574

$\begin{array}{ll}\text { Title/Name } & \text { Alberta Fishing Education Program } \\ \text { Annotation } & \begin{array}{l}\text { A factual manual covering } \\ \text { management, game fish identification, } \\ \text { fishing techniques and equipment, } \\ \text { cooking and handling the catch, safety, } \\ \text { ethical behaviour and legal } \\ \text { responsibilities in Alberta. }\end{array} \\ \text { Distributor } & \begin{array}{l}\text { Mr. Dave Paplawski or George Diduck } \\ \text { Address }\end{array} \\ & \begin{array}{l}\text { Fish and Wildlife Division } \\ \text { Conservation Education } \\ \text { 14515-122 Avenue }\end{array} \\ & \text { Edmonton, AB } \\ & \text { T5L } 2 \text { W4 }\end{array}$

\section{Author NA}

Publisher/ Alberta Forestry, Lands and

Producer Wildlife

Publication NA

Date

ISBN

Est. Cost No charge

Phone No. (403) 427-3574 or (403) 297-6423

Fax No. (403) $427-5695$ or (403) $161-2843$

or

Mr. Marty Robillard or Dave George

Fish and Wildlife Division

200 Sloan Square

59201 A Street S.W.

Calgary, $A B$

T2H OG1 
Title/Name Alberta Fish and Wildlife Conservation Education Series: Hypothermia

Annotation Well-illustrated manual available free for certified instructors. Ask about resources and instructor program. Teachers must be made aware that illustrations in this book depict male Caucasians only. The omissions of illustrations involving females and racial minorities may be offensive to some groups; teacher discretion advised. A. request will be made to the publisher to incorporate the suggestions in the next printing.

Distributor Alberta Forestry, Lands and Wildlife Address
Author

Publisher/ Alberta Forestry, Lands and

Producer Wildlife

Publication

Date

ISBN

Est. Cost No charge

Phone No. (403) 427-3574
Title/Name Alberta Fish and Wildlife Conservation Education Series: Survival

Annotation Well-illustrated manual available free for certified instructors. Ask about resources and instructor program. Teachers must be made aware that illustrations in this book depict male Caucasians only. The omissions of illustrations involving females and racial minorities may be offensive to some groups; teacher discretion advised. A request will be made to the publisher to incorporate the suggestions in the next printing.

Distributor Alberta Forestry, Lands and Wildlife Address
Fish and Wildlife Division

Conservation Education

14515-122 Avenue

Edmonton, $A B$

T5L 2W4
Author

Publisher/ Alberta Forestry, Lands and Producer Wildlife

Publication

Date

ISBN

Est. Cost No charge

Phone No. (403) 427-3574 


\section{OTHER RESOURCES}

\section{Title/Name Alberta Fish and Wildlife Conservation Education Series: Wildlife Identification \\ Annotation Well-illustrated manual available free for certified instructors. Ask about resources and instructor program. Teachers must be made aware that illustrations in this book depict male Caucasians only. The omissions of illustrations involving females and racial minorities may be offensive to some groups; teacher discretion advised. A request will be made to the publisher to incorporate the suggestions in the next printing. \\ Distributor Address \\ Alberta Forestry, Lands and Wildlife \\ Fish and Wildlife Division \\ Conservation Education \\ 14515-122 Avenue \\ Edmonton, $A B$ \\ T5L 2W4}

Author

Publlsher/

Producer

Publication

Date

ISBN

Est. Cost No charge

Phone No. (403) 427-3574 Wildlife
Alberta Forestry, Lands and
Title/Name Alberta Fish and Wildlife Conservation Education Series: Wildlife Management

Annotation Well-illustrated manual available free for certified instructors. Ask about resources and instructor program. Teachers must be made aware that illustrations in this book depict male Caucasians only. The omissions of illustrations involving females and racial minorities may be offensive to some groups; teacher discretion advised. A request will be made to the publisher to incorporate the suggestions in the next printing.

Distributor Address
Alberta Forestry, Lands and Wildlife Fish and Wildlife Division

Conservation Education 14515-122 Avenue Edmonton, $A B$

T5L 2W4

\section{Author}

Publisher/ Producer

Alberta Forestry, Lands and Wildlife

Publication

Date

ISBN

Est. Cost No charge

Phone No. (403) 427-3574 


\section{OTHER RESOURCES}

Title/Name Atlas of Environmental Issues

Annotation Describes and explains major environmental issues of today's world including soil erosion, deforestation, mechanized agriculture, oil pollution of oceans, acid rain, overfishing and nuclear power. Excellent graphics.

Distributor 4741 Queen Street Address Niagara Falls, ON L2E $2 M 2$
Author Middleton, Nick

Publisher/ Ilex Publishers Ltd.

Producer

Publication 1989

Date

ISBN $\quad 081602023 X$

Est. Cost \$21.95

Phone No. (800) 443-8323 (toll free)

Fax No. (212) 213-4578
Distributor Rocky Mountain Books

Address $\quad 106$ Wimbledon Crescent S.W. Calgary, $A B$

T3C 3J1

$\begin{array}{ll}\text { Author } & \text { Dafferin, Tony } \\ \begin{array}{l}\text { Publisher/ } \\ \text { Producer }\end{array} & \text { Rocky Mountain Books } \\ \text { Publication } & 1983 \\ \text { Date } & \\ \text { ISBN } & 0969003846\end{array}$

Est. Cost $\$ 12.95(20 \%$ discount for school libraries)

Phone No. (403) 249-2968

$\begin{array}{ll}\text { Distributor } & \text { Rocky Mountain Books } \\ \text { Address } & 106 \text { Wimbledon Crescent S.W. } \\ & \text { Calgary, AB } \\ & \text { T3C 3J1 }\end{array}$

\section{Title/Name Backcountry Biking in Canadian} Rockies

Annotation A good introduction to mountain biking and the trails in western Alberta from Waterton in the south to Jasper in the north.

106 Wimbledon Crescent S.W.

T3C 3J1

\begin{tabular}{|c|c|}
\hline Author & Lepp, Gerhard; Kerr, Colynn \\
\hline $\begin{array}{l}\text { Publisher/ } \\
\text { Producer }\end{array}$ & Rocky Mountain Books \\
\hline Publication & 1987 \\
\hline \multicolumn{2}{|l|}{ Date } \\
\hline ISBN & 0969003889 \\
\hline Est. Cost & $\begin{array}{l}\$ 12.95 \text { (20\% discount for } \\
\text { school libraries) }\end{array}$ \\
\hline Phone No. & (403) 249-2968 \\
\hline Fax No. & $\begin{array}{l}\text { (403) 249-2968 (if manual ring } \\
\text { until you hear fax tone) }\end{array}$ \\
\hline
\end{tabular}




\begin{tabular}{|c|c|c|c|}
\hline \multirow[t]{2}{*}{ Title/Name } & $\begin{array}{l}\text { Bear Attacks: Their Causes and } \\
\text { Avoidance }\end{array}$ & Author & Hererro, Stephen \\
\hline & \multirow{4}{*}{$\begin{array}{l}\text { Describes some encounters with } \\
\text { grizzly bears in the Canadian Rockies } \\
\text { and how they could have been } \\
\text { avoided. Topics such as garbage } \\
\text { disposal, bear tolerance and } \\
\text { characteristics, avoiding encounters, } \\
\text { how to recognize bear signs and bear } \\
\text { management are addressed. }\end{array}$} & $\begin{array}{l}\text { Publisher/ } \\
\text { Producer }\end{array}$ & Lyons \& Burford \\
\hline \multirow{3}{*}{ Annotation } & & $\begin{array}{l}\text { Publication } \\
\text { Date }\end{array}$ & 1988 \\
\hline & & ISBN & $\begin{array}{l}0832903779 \text { hardcover } \\
0888302797 \text { paperback }\end{array}$ \\
\hline & & Est. Cost & $\$ 13.95 / \$ 19.95$ hardcover \\
\hline \multirow{2}{*}{$\begin{array}{l}\text { Distributor } \\
\text { Address }\end{array}$} & \multirow{2}{*}{$\begin{array}{l}1302 \text { Oxford Tower } \\
\text { 10235-101 Street } \\
\text { Edmonton, AB } \\
\text { T5J 3G1 }\end{array}$} & Phone No. & (403) 426-2359 \\
\hline & & Fax No. & (403) 426-5996 \\
\hline TItle/Name & \multirow{2}{*}{$\begin{array}{l}\text { Bicycle Repair Book: Maintaining and } \\
\text { Repairing the Modern Bicycle }\end{array}$} & Author & Van de Plas, Rob \\
\hline \multirow[t]{2}{*}{ Annotation } & & $\begin{array}{l}\text { Publisher/ } \\
\text { Producer }\end{array}$ & Bicycle Books Inc. \\
\hline & $\begin{array}{l}\text { An excellent manual of complete } \\
\text { bicycle care. The main focus of this } \\
\text { book is on the touring style of bicycle. }\end{array}$ & Publication & 1989 \\
\hline \multirow{5}{*}{$\begin{array}{l}\text { Distributor } \\
\text { Address }\end{array}$} & \multirow{5}{*}{$\begin{array}{l}112 \text { East 3rd Avenue } \\
\text { Vancouver, BC } \\
\text { V5T } 1 \mathrm{C} 8\end{array}$} & Date & \\
\hline & & ISBN & 0933201117 \\
\hline & & Est. Cost & $\$ 11.95$ \\
\hline & & Phone No. & (604) 873-6581 \\
\hline & & Fax No. & (604) 874-2711 \\
\hline Title/Name & Canoeing & Author & Kelba, N. et al. \\
\hline Annotation & $\begin{array}{l}\text { One of } 10 \text { recommended support } \\
\text { modules in the "Peer Booklet Series." }\end{array}$ & $\begin{array}{l}\text { Publisher/ } \\
\text { Producer }\end{array}$ & Calgary Board of Education \\
\hline \multirow{6}{*}{$\begin{array}{l}\text { Distributor } \\
\text { Address }\end{array}$} & \multirow{6}{*}{$\begin{array}{l}\text { Calgary Board of Education } \\
\text { Physical Education Department } \\
515 \text { MacLeod Trail S.E. } \\
\text { Calgary, AB } \\
\text { T2G 2L9 }\end{array}$} & Publication & 1980 \\
\hline & & Date & \\
\hline & & ISBN & \\
\hline & & Est. Cost & \\
\hline & & Phone No. & (403) 294-8206 \\
\hline & & Fax No. & (403) 294-8336 \\
\hline
\end{tabular}




$\begin{array}{ll}\text { Title/Name } & \text { Canoeing Alberta } \\ \text { Annotation } & \begin{array}{l}\text { Descriptions, maps, information } \\ \text { regarding six different river systems in } \\ \text { Alberta. Also addresses topics such as } \\ \text { understanding rivers, personal safety, } \\ \text { leader safety and grading rivers. }\end{array} \\ \text { Distributor } & \text { Lone Pine Publishing } \\ \text { Address } & \text { Suite 206, 10426-81 Avenue } \\ & \text { Edmonton, AB } \\ & \text { T6E 1X5 }\end{array}$
Author
Macdonald, Janice E.
Publisher/ Lone Pine Publishing
Producer
Publication 1985
Date
ISBN 0919433286
Est. Cost $\$ 11.95+\$ 1$ shipping and handling

Phone No. (403) 433-9333

Fax No.

\begin{tabular}{|c|c|}
\hline Title/Name & $\begin{array}{l}\text { Challenge Program } \\
\text { Cross-Country Program for Teens }\end{array}$ \\
\hline Annotation & $\begin{array}{l}\text { Challenge Program (13 to } 17 \text { years) } \\
\text { Manuals } \$ 18 \\
\text { "Startup Package } \$ 30 \\
\text { Includes one manual, five posters, two } \\
\text { challenge crests, registration with } \\
\text { Cross-Country Alberta and Cross- } \\
\text { Country Canada and subscription to } \\
\text { Challenge newsletter. }\end{array}$ \\
\hline $\begin{array}{l}\text { Distributor } \\
\text { Address }\end{array}$ & $\begin{array}{l}11759 \text { Groat Road } \\
\text { Edmonton, AB } \\
\text { T5M 3K6 }\end{array}$ \\
\hline Title/Name & Conceptual Encounters II \\
\hline Annotation & $\begin{array}{l}\text { Focuses on developing a single } \\
\text { ecological concept, in increments, } \\
\text { flowing in small steps from the } \\
\text { concrete to the abstract level of } \\
\text { understanding. } \\
\text { Each activity requires } 1 \text { to } 1.5 \text { hours to } \\
\text { complete. Ideal for field trip } \\
\text { experiences or nature centre visits. } \\
\text { Appropriate up to end of Grade } 9 . \\
\text { (Part of Sunship III Program.) }\end{array}$ \\
\hline $\begin{array}{l}\text { Distributor } \\
\text { Address }\end{array}$ & $\begin{array}{l}\text { P.O. Box } 3444, \text { Station G } \\
\text { Calgary, AB } \\
\text { T3A } 2 \text { G6 }\end{array}$ \\
\hline
\end{tabular}

$\begin{array}{ll}\begin{array}{l}\text { Author } \\ \text { Publisher/ } \\ \text { Producer }\end{array} & \text { Landry, L. } \\ \text { Publication } & 1988 \\ \text { Date } & \\ \text { ISBN } & \text { NA } \\ \text { Est. Cost } & \text { shown } \\ \text { Phone No. } & \text { (403) 453-8620 } \\ \text { Fax No. } & \text { (403) 453-8553 } \\ & \end{array}$

Author

Institute for Earth Education

Publisher/ Institute for Earth Education

Producer

Publication To be published late 1990

Date

ISBN

TBA

Est. Cost approx. $\$ 57.45$

Phone No. (403) 246-6611

Fax No. (403) 294-8336 Attn: Shirley Mitchell 


\begin{tabular}{|c|c|c|c|}
\hline Title/Name & Cowstails and Cobras $\|$ & Author & Rohnke, Karl \\
\hline \multirow[t]{5}{*}{ Annotation } & \multirow{5}{*}{$\begin{array}{l}\text { A revision of Karl Rohnke's classic } \\
\text { guide to games, initiative problems and } \\
\text { adventure activities. Updated and now } \\
\text { includes sample curriculum (actual } \\
\text { program outlines from schools and } \\
\text { camps) and a chapter on leadership } \\
\text { and debriefing. Offering a thorough } \\
\text { treatment of Project Adventure's } \\
\text { philosophy and approach to group } \\
\text { activities, Cowstails II provides both the } \\
\text { experienced practitioner and the novice } \\
\text { with a unique and valuable resource. } \\
\text { With photos and illustrations. }\end{array}$} & $\begin{array}{l}\text { Publisher/ } \\
\text { Producer }\end{array}$ & Project Adventure \\
\hline & & $\begin{array}{l}\text { Publication } \\
\text { Date }\end{array}$ & NA \\
\hline & & ISBN & $084035434 X$ \\
\hline & & Est. Cost & $\begin{array}{l}\$ 16.50+\$ 2 \text { shipping and } \\
\text { handling }\end{array}$ \\
\hline & & $\begin{array}{l}\text { Phone No. } \\
\text { Fax No. }\end{array}$ & $(508) 468-7981$ \\
\hline $\begin{array}{l}\text { Distributor } \\
\text { Address }\end{array}$ & $\begin{array}{l}\text { Project Adventure } \\
\text { P.O. Box } 100 \\
\text { Hamilton, MA } \\
01936 \\
\text { U.S.A. }\end{array}$ & & \\
\hline Title/Name & Diet For a New America & Author & Robbins, John \\
\hline \multirow[t]{2}{*}{ Annotation } & \multirow{2}{*}{$\begin{array}{l}\text { Graphic description of current food } \\
\text { processing procedures and how food } \\
\text { choices affect your health, happiness } \\
\text { and the future of life on earth. }\end{array}$} & $\begin{array}{l}\text { Publisher/ } \\
\text { Producer }\end{array}$ & Stillpoint Publishing \\
\hline & & Publication & 1987 \\
\hline \multirow{5}{*}{$\begin{array}{l}\text { Distributor } \\
\text { Address }\end{array}$} & \multirow{5}{*}{$\begin{array}{l}195 \text { Allstate Parkway } \\
\text { Markham, ON } \\
\text { L3R 4T8 }\end{array}$} & Date & \\
\hline & & ISBN & 0913299545 \\
\hline & & Est. Cost & $\$ 17.95$ \\
\hline & & Phone No. & (416) 477-0030 \\
\hline & & Fax No. & (416) 477-9179 \\
\hline
\end{tabular}




\begin{tabular}{|c|c|c|c|}
\hline \multirow{2}{*}{$\begin{array}{l}\text { Title/Name } \\
\text { Annotation }\end{array}$} & \multirow{2}{*}{$\begin{array}{l}\text { Duke of Edinburgh Award in Canada: } \\
\text { Handbook }\end{array}$} & Author & NA \\
\hline & & $\begin{array}{l}\text { Publisher/ } \\
\text { Producer }\end{array}$ & $\begin{array}{l}\text { Duke of Edinburgh Award in } \\
\text { Canada }\end{array}$ \\
\hline \multirow{3}{*}{. } & \multirow{3}{*}{$\begin{array}{l}\text { the areas of volunteer community } \\
\text { service, expeditions, hobby/skills and } \\
\text { physical recreation. Students } 14 \text { to } 25 \\
\text { years, able or disabled, can register } \\
\text { and qualify for three levels of awards: } \\
\text { Bronze, Silver and Gold. }\end{array}$} & $\begin{array}{l}\text { Publication } \\
\text { Date }\end{array}$ & 1989 \\
\hline & & ISBN & 0905425014 \\
\hline & & Est. Cost & NA \\
\hline \multirow[t]{3}{*}{$\begin{array}{l}\text { Distributor } \\
\text { Address }\end{array}$} & \multirow{3}{*}{$\begin{array}{l}11759 \text { Groat Road } \\
\text { Edmonton, Ab } \\
\text { T5M } 3 \mathrm{~K} 6 \\
\text { or } \\
\text { Suite } 010,611-10 \text { Avenue S.W. } \\
\text { Calgary, AB } \\
\text { T2R OB2 }\end{array}$} & Phone No. & $\begin{array}{l}\text { (403) } 453-8651 \text { or } \\
\text { (403) } 237-7476\end{array}$ \\
\hline & & Fax No. & $\begin{array}{l}\text { (403) } 453-8553 \text { or } \\
(403) 237-7476\end{array}$ \\
\hline & & & \\
\hline Title/Name & Earth Speaks, The & Author & Institute for Earth Education \\
\hline \multirow[t]{2}{*}{ Annotation } & \multirow{2}{*}{$\begin{array}{l}\text { A collection of excellent literary works } \\
\text { by some of the world's most respected } \\
\text { authors, poets, ecologists and } \\
\text { environmentalists. }\end{array}$} & $\begin{array}{l}\text { Publisher/ } \\
\text { Producer }\end{array}$ & Institute for Earth Education \\
\hline & & Publication & \\
\hline \multirow{5}{*}{$\begin{array}{l}\text { Distributor } \\
\text { Address }\end{array}$} & \multirow{5}{*}{$\begin{array}{l}\text { P.O. Box } 880 \text {, Station } G \\
\text { Calgary, AB } \\
\text { T3A } 2 \text { G6 }\end{array}$} & Date & \\
\hline & & ISBN & 0917001007 \\
\hline & & Est. Cost & $\$ 15.95$ \\
\hline & & Phone No. & (403) $246-6611$ \\
\hline & & Fax No. & $\begin{array}{l}\text { (403) 294-8336 Attn: Shirley } \\
\text { Mitchell }\end{array}$ \\
\hline
\end{tabular}




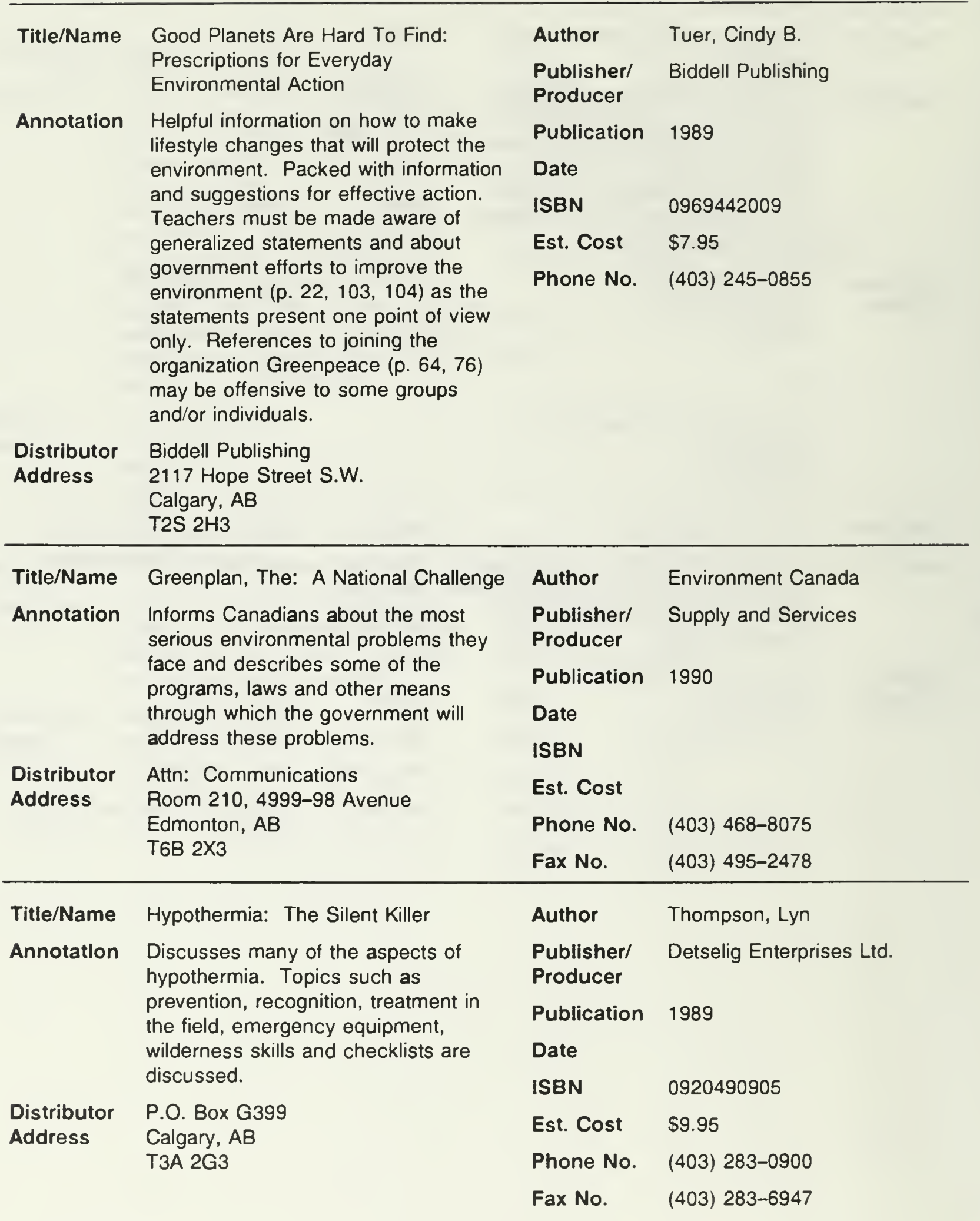


Title/Name Investigating and Evaluating

Environmental Issues and Actions:

Skill Development Modules: A

Curriculum Development Project

Designed to Teach Students How to

Investigate and Evaluate Science-

Related Social Issues

Annotation Outlines process for building analytic and decision-making skills. Appropriate for advanced students. Teacher's guide available for \$23.95-details in library list. Teachers must be made aware that the "Comments on Values and Values Clarification" statement on yellow pages 15 and 16 is contrary to Alberta Education's Controversial Issues Policy. This policy implies that it is not the teacher's task to take sides, to impose personal views or to resolve the world's problems. It is advised that teachers not use yellow pages 15 and 16 as reference material.
Author

Publisher/

Producer

Publication 1988

Date

ISBN

Est. Cost

Phone No.
Hungerford, $H$. R. et al.

Stipes Publishing Co.

$087563320 x$

$\$ 18.10$

(416) 593-0737

\section{Distributor Can-Ed. Media Ltd. \\ Address 185 Spadina Avenue \\ Toronto, ON \\ M5T 2 C6}

Title/Name Investigating and Evaluating

Environmental Issues and Actions:

Teacher's Edition

Annotation Teacher's guide to accompany student modules listed as support resources.

Distributor

Address
Can-Ed. Media Ltd.

185 Spadina Avenue

Toronto, ON

M5T 2 C6
Author
Hungerford, Harold
Publisher/
Stipes Publishing Co.
Producer
Publication 1988
Date
ISBN
087563320X
Est. Cost $\$ 23.95$
Phone No. (416) 593-0737 


\begin{tabular}{|c|c|c|c|}
\hline Title/Name & Investigating Aquatic Ecosystems & Author & Andrews, William \\
\hline \multirow[t]{5}{*}{ Annotation } & \multirow{5}{*}{$\begin{array}{l}\text { Leads to an understanding of the } \\
\text { principles of ecology. Students can } \\
\text { start asking questions such as: Why } \\
\text { are trout no longer found in a once } \\
\text { favourable trout stream? What can be } \\
\text { done to restore favourable conditions? } \\
\text { What are the effects for damming a } \\
\text { river to provide an artificial lake for } \\
\text { recreational purposes? }\end{array}$} & $\begin{array}{l}\text { Publisher/ } \\
\text { Producer }\end{array}$ & Prentice-Hall \\
\hline & & $\begin{array}{l}\text { Publication } \\
\text { Date }\end{array}$ & 1987 \\
\hline & & ISBN & $013503129 x$ \\
\hline & & Est. Cost & $\$ 29.35$ \\
\hline & & Phone No. & (416) 293-3621 \\
\hline $\begin{array}{l}\text { Distributor } \\
\text { Address }\end{array}$ & $\begin{array}{l}\text { Prentice-Hall } \\
1870 \text { Birchmount Road } \\
\text { Scarborough, ON } \\
\text { M1P 2J7 }\end{array}$ & & \\
\hline Title/Name & Investigating Terrestrial Ecosystems & Author & Andrews, William \\
\hline \multirow[t]{5}{*}{ Annotation } & \multirow{5}{*}{$\begin{array}{l}\text { Deals with interrelationships found in } \\
\text { forests, woodlots, meadows, parks, } \\
\text { bogs, sand dunes and other land-based } \\
\text { ecological systems. It provides } \\
\text { background and the procedures } \\
\text { required to investigate such } \\
\text { relationships in the laboratory and in } \\
\text { the field. }\end{array}$} & $\begin{array}{l}\text { Publisher/ } \\
\text { Producer }\end{array}$ & Prentice-Hall \\
\hline & & Publication & 1986 \\
\hline & & Date & \\
\hline & & ISBN & 0135031869 \\
\hline & & Est. Cost & $\$ 28.18$ \\
\hline $\begin{array}{l}\text { Distributor } \\
\text { Address }\end{array}$ & $\begin{array}{l}\text { Prentice-Hall } \\
1870 \text { Birchmount Road } \\
\text { Scarborough, ON } \\
\text { M1P 2J7 }\end{array}$ & Phone No. & (416) 293-3621 \\
\hline \multirow[t]{2}{*}{ TItle/Name } & \multirow{2}{*}{$\begin{array}{l}\text { Jackrabbit Ski League/Lique de Ski } \\
\text { Jackrabbit Skill Development Program }\end{array}$} & Author & Landry, L. \\
\hline & & Publisher/ & Cross-Country Canada \\
\hline \multirow{4}{*}{ Annotation } & \multirow{3}{*}{$\begin{array}{l}\text { Cross-country ski program for } 8 \text { to } 13 \\
\text { years old. Available in English or } \\
\text { French. }\end{array}$} & Producer & \\
\hline & & Publication & 1987 \\
\hline & & Date & \\
\hline & $\begin{array}{l}\text { Manual \$15 } \\
\text { Games Manual \$2 }\end{array}$ & ISBN & NA \\
\hline \multirow{3}{*}{$\begin{array}{l}\text { Distributor } \\
\text { Address }\end{array}$} & \multirow{3}{*}{$\begin{array}{l}11759 \text { Groat Road } \\
\text { Edmonton, AB } \\
\text { T5M 3K6 }\end{array}$} & Est. Cost & shown \\
\hline & & Phone No. & (403) 453-8620 \\
\hline & & Fax No. & (403) 453-8553 \\
\hline
\end{tabular}




\section{OTHER RESOURCES}

\begin{tabular}{|c|c|c|c|}
\hline Title/Name & Kananaskis Trail Guide & Author & Daffern, Gillian \\
\hline Annotation & $\begin{array}{l}\text { Comprehensive guide to hiking and } \\
\text { skiing trails in Kananaskis Country. }\end{array}$ & $\begin{array}{l}\text { Publisher/ } \\
\text { Producer }\end{array}$ & Rocky Mountain Books \\
\hline \multirow[t]{5}{*}{$\begin{array}{l}\text { Distributor } \\
\text { Address }\end{array}$} & \multirow[t]{5}{*}{$\begin{array}{l}106 \text { Wimbledon Crescent S.W. } \\
\text { Calgary. AB } \\
\text { T3C 3J1 }\end{array}$} & $\begin{array}{l}\text { Publication } \\
\text { Date }\end{array}$ & 1985 \\
\hline & & ISBN & \\
\hline & & Est. Cost & $\begin{array}{l}\$ 12.95 \text { (20\% discount for } \\
\text { school libraries) }\end{array}$ \\
\hline & & Phone No. & (403) 249-2968 \\
\hline & & Fax No. & $\begin{array}{l}\text { (403) } 249-2968 \text { (if manual ring } \\
\text { until you hear fax tone) }\end{array}$ \\
\hline Title/Name & \multirow{2}{*}{$\begin{array}{l}\text { Keepers of the Earth } \\
\text { Keepers of the Earth: Teacher's Guide }\end{array}$} & Author & Caduto, M. and Bruhac, J. \\
\hline \multirow[t]{2}{*}{ Annotation } & & $\begin{array}{l}\text { Publlsher/ } \\
\text { Producer }\end{array}$ & Fifth House Publishers \\
\hline & $\begin{array}{l}\text { Native American stories and } \\
\text { environmental activities for students. }\end{array}$ & Publlcation & 1989 (book) 1990 (guide) \\
\hline \multirow[t]{4}{*}{$\begin{array}{l}\text { Distributor } \\
\text { Address }\end{array}$} & $\begin{array}{l}\text { 20-36 Street East } \\
\text { Saskatoon, SK }\end{array}$ & Date & \\
\hline & S7K 5S8 & ISBN & $\begin{array}{l}0920079571 \text { (book) } \\
1555910408 \text { (guide) }\end{array}$ \\
\hline & & Est. Cost & $\$ 22.95$ (book) \\
\hline & & Phone No. & NA \\
\hline
\end{tabular}

\section{TItle/Name Learning Resources Manual for} Alberta's Provincial Parks

Annotation Supports grades 1 to 9 curricula. The manual plus an additional chapter for most provincial parks are available. A new manual to complement the new junior high curriculum is being developed.

Distributor Environmental Education

Address Brian Ogston, Co-ordinator 7th Floor, Standard Life Centre 10405 Jasper Avenue Edmonton, $A B$

T5J 3N4
Author

Publisher/

Producer

Publication

Date

ISBN

Est. Cost

Manual $\$ 15$

Park Chapters \$1 each

Phone No. (403) 427-8758

Fax No. $\quad$ (403) 424-9492 


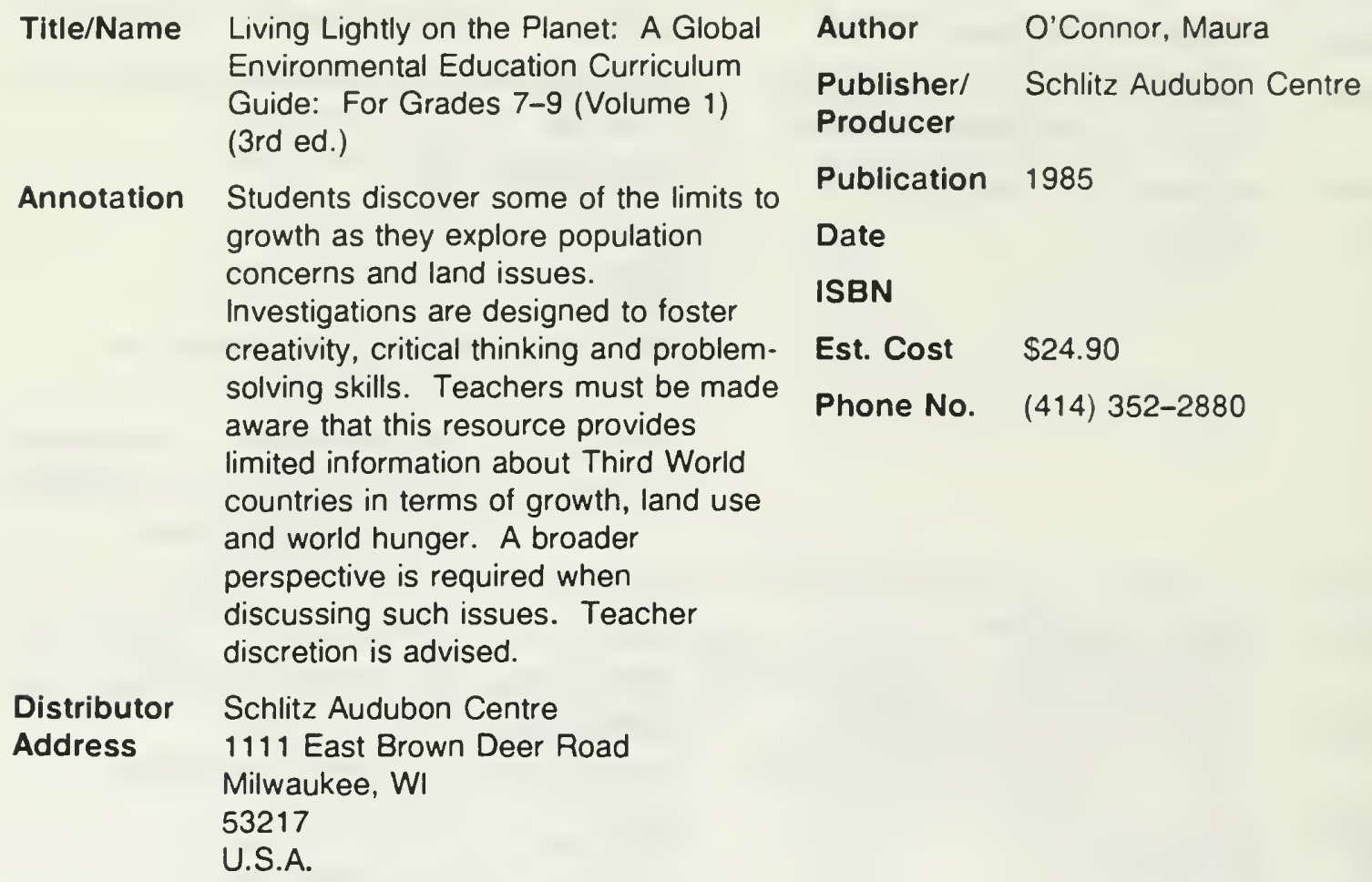

Title/Name Living Lightly on the Planet: A Global Environmental Education Curriculum Guide: For Grades 7-9 (Volume 1) (3rd ed.)

Annotation Students discover some of the limits to growth as they explore population concerns and land issues. Investigations are designed to foster creativity, critical thinking and problemsolving skills. Teachers must be made aware that this resource provides limited information about Third World countries in terms of growth, land use and world hunger. A broader perspective is required when discussing such issues. Teacher discretion is advised.

Distributor

Schlitz Audubon Centre

1111 East Brown Deer Road

Milwaukee, WI

53217

U.S.A.

Author

Publisher/

Producer

Publication

Date

ISBN

Est. Cost $\$ 24.90$

Phone No. (414) 352-2880

O'Connor, Maura

Schlitz Audubon Centre

1985

\begin{tabular}{|c|c|}
\hline Title/Name & Medicine for Mountaineering \\
\hline Annotation & $\begin{array}{l}\text { A resource text (as opposed to a } \\
\text { manual) that discusses general } \\
\text { principles, traumatic injuries, } \\
\text { environmental injuries and non- } \\
\text { traumatic diseases from a backcountry } \\
\text { point of view. }\end{array}$ \\
\hline $\begin{array}{l}\text { Distributor } \\
\text { Address }\end{array}$ & $\begin{array}{l}\text { Education Division } \\
1615 \text { Venables Street } \\
\text { Vancouver, BC } \\
\text { V5L } 2 \mathrm{H} 1\end{array}$ \\
\hline
\end{tabular}

\section{Author Wilkerson, James A. \\ Publisher/ The Mountaineers Producer \\ Publication \\ Date \\ ISBN \\ 0898860865}

Est. Cost $\$ 17.95$

Phone No. (604) 254-7191

Fax No. (604) 254-9099 
Title/Name More New Games Book

Annotation Playful ideas from the New Games

Foundation. More ways for everyone

to play. "Rules" for 60 new games (for two to 200 people). A valuable resource for many groups and events, schools, parks and playgrounds, company picnics, family reunions you name it!

Distributor Special Sales

Address 105 Bond Street

Toronto, ON M5B 1 Y3
Author

Fluegelman, Andrew

Publisher/ Headlands Press

Producer

Publication 1981

Date

ISBN

0385175140

Est. Cost

$\$ 15.95$

Phone No. (416) 340-0777

Fax No. (416) 340-1069
TItle/Name Mountaineering First Aid: A Guide to Accident Response and First Aid Care

Annotation A practical guide to backcountry emergency management. Topics such as before you go, when an emergency happens, first aid for specific conditions, evacuation techniques and emergency plans are covered.

Distributor Educational Division

Address $\quad 1615$ Venables Street

Vancouver, BC V5L $2 \mathrm{H}_{1}$
Author

Publisher/ The Mountaineers

Producer

Publication 1985

Date

ISBN 089886092X

Est. Cost $\$ 8.50$

Phone No. (604) 254-7191

Fax No. (604) 254-9099
Title/Name Mountaineering: The Freedom of the Hills (3rd ed.)

Annotation Basic essentials to the backcountry experience are discussed. Topics such as preparation, equipment, clothing, navigation, route finding, rock climbing, snow and ice climbing techniques, safety concerns and the environment are presented in depth.

Distributor Douglas \& Mclntyre Address 1615 Venables Street Vancouver, BC V5L $2 \mathrm{H} 1$
Author Ferber, P. (ed.)

Publisher/ The Mountaineers Producer

Publlcation 1974

Date

ISBN 0916890015

Est. Cost $\$ 24.50$

Phone No. (604) 254-7191

Fax No. (604) 254-9099 


\begin{tabular}{|c|c|}
\hline Title/Name & $\begin{array}{l}\text { Natural Regions of Alberta: Poster } \\
\text { Series: Resource Manual }\end{array}$ \\
\hline Annotation & $\begin{array}{l}\text { Contains a poster for each of five } \\
\text { major natural regions of Alberta } \\
\text { accompanied by a comprehensive } \\
\text { teacher's resource manual. The } \\
\text { manual includes student activity } \\
\text { suggestions, grades } 1 \text { through } 9 \text {. }\end{array}$ \\
\hline $\begin{array}{l}\text { Distributor } \\
\text { Address }\end{array}$ & $\begin{array}{l}\text { Alberta Recreation and Parks } \\
7 \text { th Floor, Standard Life Centre } \\
10405 \text { Jasper Avenue } \\
\text { Edmonton, AB } \\
\text { T5J 3N4 }\end{array}$ \\
\hline Title/Name & New Games Book, The \\
\hline Annotation & $\begin{array}{l}\text { A collection of games that emphasize a } \\
\text { co-operative approach to enjoying } \\
\text { group activities. Games are designed } \\
\text { for two to } 100 \text { people. The games } \\
\text { involve very little equipment. Teachers } \\
\text { must be made aware that some } \\
\text { students and community members may } \\
\text { not feel comfortable with and/or } \\
\text { approve of the close physical proximity } \\
\text { among participants required for several } \\
\text { games (e.g., p. } 40,57,97,101,116 \text {, } \\
117,151,157,170) \text {. It is } \\
\text { recommended that teachers carefully } \\
\text { select appropriate games. }\end{array}$ \\
\hline $\begin{array}{l}\text { Distributor } \\
\text { Address }\end{array}$ & $\begin{array}{l}\text { Doubleday Canada, Special Sales } \\
105 \text { Bond Street } \\
\text { Toronto, ON } \\
\text { M5B } 1 \text { Y3 }\end{array}$ \\
\hline
\end{tabular}

Author

Publisher/ Alberta Recreation and Parks

Producer

Publication

Date

ISBN

Est. Cost $\$ 20$

Phone No. (403) 427-8758

\section{Author New Games Foundation \\ Publisher/ The Headlands Press Producer \\ Publication 1976 \\ Date \\ ISBN 038512516X}

Est. Cost $\$ 10.85$

Phone No. (416) 977-7891 


\section{Title/Name Northern Alberta Environmental \\ Education Program: Level 2: Grades 7 to 9}

Annotation The program is divided into two levels. The Level 1 binder deals with the scientific background students require to understand environmental issues while the Level 2 binder deals with the environmental issues per se. Level 1 and Level 2 are available separately.

Distributor Friends of Environmental Education Address
Society of Alberta

9914-76 Avenue

Edmonton, $A B$

T6E 1K8
Author

Publisher/ Friends of Environmental

Producer Education Society of Alberta (FEESA)

Publication 1989

Date

ISBN

Est. Cost $\$ 95$ complete program

Phone No. (403) 439-0243

Fax No. (403) 432-1203
Title/Name Operation Lifeline

Annotation Kit includes one poster, 10 Lifeline newsletters, one activity book and one teacher's guide. Available in English and French. The program stimulates and awakens interest in Canadian endangered species, their habitats, environment and fragile life support systems.

W36752 Operation Lifeline W25763 Opération Survie

Distributor SeeHearNow! Media

Address $\quad 3771$ Victoria Park Avenue

Scarborough, ON M1W 2P9

\section{Author}

Publisher/

Producer

Publication

Date

ISBN

Est. Cost

Phone No.
World Wildlife Fund (Canada)

The Canada Life Assurance Co.

1986

NA

$\$ 29.95$

(800) 263-4005
Author and Recreation Programs

Annotation An overview of considerations for outdoor program development and associated outdoor pursuit activities. It contains a good section on initiative games.

$\begin{array}{ll}\text { Distributor } & 35 \text { Cobequid Drive } \\ \text { Address } & \text { Truro, NS } \\ & \text { B2N 5R1 }\end{array}$

\section{Publisher/ Burgess Pub. Co.} Producer

Publication 1980

Date

ISBN 0808704893

Est. Cost \$19.95

Phone No. (902) 893-1057

Fax No. (902) 893-1464 
Title/Name Peer Support: Designing Interpersonal Author Skills: A Training Plan

Annotation Series of activities that encourage the development of positive identity and responsible independence as individuals exercise more control over their experiences. Uses a structured interpersonal skill training base to assist in the development of positive peer relations and encourages the use of peer group to support self-directed learning. Teachers must be made aware that the adaptation from the "Values Clarification" on p. 62 is contrary to Alberta Education's Controversial Issues Policy.

Program Plan $\$ 20$

Teacher's Resource $\$ 40$

\section{Distributor AADAC}

Address

Product and Distribution Branch

2nd Floor, 10909 Jasper Avenue

Edmonton, $A B$

T5J $3 \mathrm{M} 9$
Davis, 8.; Handcock, H.; Hills, $M$.

Publisher/

AADAC

Producer

Publication

1987

Date

ISBN

Est. Cost Free from AADAC

Phone No. (403) 427-7319

$\begin{array}{ll}\text { Title/Name } & \begin{array}{l}\text { Prospectus for an Alberta Conse } \\ \text { Strategy }\end{array} \\ \text { Annotation } & \begin{array}{l}\text { Public Advisory Committee repo } \\ \text { the Environment Council of Albe }\end{array} \\ & \text { Available free on request. } \\ \text { Distributor } & \begin{array}{l}\text { Environment Council of Alberta } \\ \text { Address }\end{array} \\ & \begin{array}{l}\text { 8th Floor Weber Building } \\ 5555 \text { Calgary Trail Southbound } \\ \text { Edmonton, AB }\end{array} \\ & \text { T6H 5P9 }\end{array}$

Title/Name

Prospectus for an Alberta Conservation
Public Advisory Committees to the Environment Council of Alberta

Publisher/ Environment Council of Alberta Producer

Publication 1986

Date

ISBN

Est. Cost No charge direct

Phone No. (403) 427-5792 


\section{OTHER RESOURCES}

\begin{tabular}{|c|c|c|c|}
\hline Title/Name & School Ski Program & Author & Landry, L. \\
\hline & Participation, Games and Skills, Fitness & $\begin{array}{l}\text { Publisher/ } \\
\text { Producer }\end{array}$ & Cross-Country Canada \\
\hline & & Publication & 1987 \\
\hline & $\begin{array}{l}\text { \#tartup package } \$ 88 \\
\text { Manual } \$ 25\end{array}$ & Date & \\
\hline & Video $\$ 35$ & ISBN & NA \\
\hline & Startuo packao & Est. Cost & shown \\
\hline & 36 certificates, 36 games and skill & Phone No. & (403) 453-8620 \\
\hline & $\begin{array}{l}\text { decals, one chart, one poster, one } \\
\text { video (A Skiing Experience). }\end{array}$ & Fax No. & (403) 453-8553 \\
\hline $\begin{array}{l}\text { Distributor } \\
\text { Address }\end{array}$ & $\begin{array}{l}11759 \text { Groat Road } \\
\text { Edmonton, AB } \\
\text { T5M 3K6 }\end{array}$ & & \\
\hline
\end{tabular}

Title/Name Sharing Nature with Children: The Classic Parents' and Teachers' Nature Awareness Guidebook

Annotation A collection of simple activities that stress environmental education. The activities are easily adopted for site, grade concept and timeframe.

$\begin{array}{ll}\text { Distributor } & \text { P.O. Box } 698 \\ \text { Address } & \text { Markdale, ON } \\ & \text { NOC } 1 \mathrm{HO}\end{array}$

Distributor P.O. Box 698

NOC $1 \mathrm{HO}$

$\begin{array}{ll}\text { Author } & \text { Cornell, Joseph } \\ \text { Publisher/ } & \text { Crystal Clarity } \\ \text { Producer } & \\ \text { Publication } & 1979 \\ \text { Date } & \end{array}$

ISBN

0916124142

Est. Cost $\$ 9.75$

Phone No. (519) 986-4353

Fax No. (519) $986-4353$
Title/Name Ski Camping

Annotation An excellent guide to ski camping including equipment, techniques and safety. There are particularly useful checklists and charts.

$\begin{array}{ll}\text { Distributor } & \text { Douglas \& Mclntyre } \\ \text { Address } & 1615 \text { Venables Street } \\ & \text { Vancouver, BC } \\ & \text { V5L } 2 \mathrm{H} 1\end{array}$

$\begin{array}{ll}\text { Author } & \text { Watters, R. } \\ \text { Publisher/ } & \text { North Country Book Express } \\ \text { Producer } & \\ \text { Publication } & 1979 \\ \text { Date } & \\ \text { ISBN } & 088942567\end{array}$

Est. Cost

Phone No. (604) 254-7191

Fax No. (604) 254-9099 


\begin{tabular}{|c|c|c|c|}
\hline \multirow[t]{2}{*}{ Title/Name } & \multirow{2}{*}{$\begin{array}{l}\text { State of the Ark: An Atlas of } \\
\text { Conservation in Action }\end{array}$} & Author & Durrell, Lee \\
\hline & & Publisher/ & Doubleday \\
\hline \multirow[t]{6}{*}{ Annotation } & \multirow{6}{*}{$\begin{array}{l}\text { Presents the Ark as the self-sustaining } \\
\text { biosphere and its inhabitants as a great } \\
\text { range of living species, both plant and } \\
\text { animal. Deals with the state of natural } \\
\text { ecosystems, species survival and the } \\
\text { world's main regions. The rise of the } \\
\text { conservation movement is discussed } \\
\text { as are suggestions for corrective } \\
\text { action. }\end{array}$} & Producer & \\
\hline & & Publication & 1986 \\
\hline & & Date & \\
\hline & & ISBN & 0385236689 \\
\hline & & Est. Cost & $\$ 22.95$ \\
\hline & & Phone No. & (416) 340-0777 \\
\hline $\begin{array}{l}\text { Distributor } \\
\text { Address }\end{array}$ & $\begin{array}{l}105 \text { Bond Street } \\
\text { Toronto, ON } \\
\text { M5B } 1 \text { Y3 }\end{array}$ & Fax No. & (416) 340-1069 \\
\hline Title/Name & Sunship III & Author & Institute for Earth Education \\
\hline \multirow[t]{2}{*}{ Annotation } & \multirow{2}{*}{$\begin{array}{l}\text { Available Spring 1991. Part of the } \\
\text { Conceptual Encounters Program, it is a } \\
\text { teacher's guide for running the } \\
\text { program. }\end{array}$} & $\begin{array}{l}\text { Publisher/ } \\
\text { Producer }\end{array}$ & Institute for Earth Education \\
\hline & & Publication & Tentatively 1991 \\
\hline \multirow{5}{*}{$\begin{array}{l}\text { Distributor } \\
\text { Address }\end{array}$} & \multirow{5}{*}{$\begin{array}{l}\text { P.O. Box } 880, \text { Station } G \\
\text { Calgary, } A B \\
\text { T3A } 2 \text { G6 }\end{array}$} & Date & \\
\hline & & ISBN & TBA \\
\hline & & Est. Cost & TBA \\
\hline & & Phone No. & (403) 246-6611 \\
\hline & & Fax No. & $\begin{array}{l}\text { (403) 294-8336 Attn: Shirley } \\
\text { Mitchell }\end{array}$ \\
\hline Title/Name & $\begin{array}{l}\text { Trapping and Conservation Program } \\
\text { Manual }\end{array}$ & Author & $\begin{array}{l}\text { Alberta Vocational Centre Lac } \\
\text { La Biche, E. Robertson et al. }\end{array}$ \\
\hline \multirow[t]{4}{*}{ Annotation } & \multirow{4}{*}{$\begin{array}{l}\text { Comprehensive manual providing } \\
\text { information about trapping wild fur- } \\
\text { bearing animals. Deals with capture } \\
\text { methods, fur handling and } \\
\text { management. A condensed version of } \\
\text { this manual is expected to be available } \\
\text { in } 1991 \text {. }\end{array}$} & $\begin{array}{l}\text { Publisher/ } \\
\text { Producer }\end{array}$ & \\
\hline & & Publication & 1987 \\
\hline & & Date & \\
\hline & & ISBN & NA \\
\hline Distributor & Fur Management Council & Est. Cost & $\mathrm{N} / \mathrm{C}$ \\
\hline Address & $\begin{array}{l}\text { Fred Neumann } \\
\text { Fur Management Co-ordinator } \\
\text { Main Office, North Tower } \\
\text { Petroleum Plaza } \\
9945-108 \text { Street } \\
\text { Edmonton, AB } \\
\text { T5K 2G6 }\end{array}$ & Phone No. & (403) 427-6750 \\
\hline
\end{tabular}


Title/Name Water Quality Questions: The Nature and Importance of Water Quality Variables in Alberta (Text, Teacher's Guide)

Annotation Introduces students to the physical and ecological characteristics of a river, the various ways humans have an impact on rivers and the means available to monitor and manage river water quantity and quality.

Distributor Environmental Education Branch Address

Water Literacy Program 9820-106 Street

Edmonton, $A B$

T5K 2J6
Author Stoker, D.

Publisher/ Alberta Environment

Producer

Publication 1989

Date

ISBN

Est. Cost Free with Workshop

Phone No. (403) 427-6310
Title/Name Wholistic Leadership Development Model (Used to design Blue Lake Centre Youth Leadership Seminar)

Annotation Wholistic leadership occurs when an individual, a group or an organization intentionally influences the behaviour, attitudes and values of another individual, group or organization. Useful to teachers concerned with environmental education.

Distributor Attn: Lyle Benson

Address 97 Cheviot Drive

Hinton, $A B$

T7V 1R1

\section{Author Benson, Lyle \\ Publisher/ Leadership Associates Producer}

Publication Early 1991 (estimated)

Date

ISBN TBA

Est. Cost \$25

Phone No. (403) 865-4712

Fax No. (403) 865-1167 
•

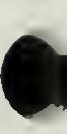



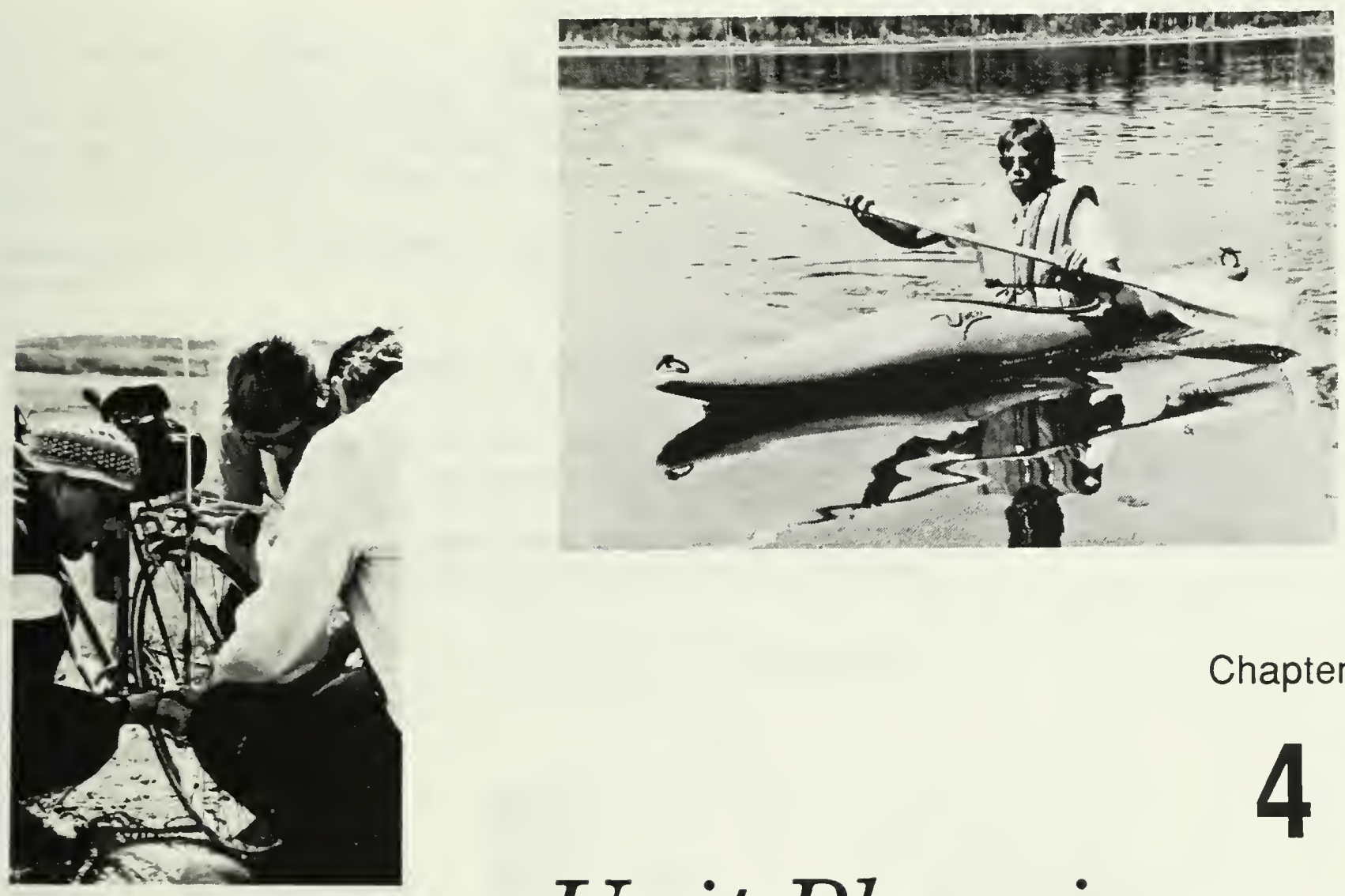

Chapter

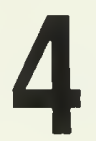

\section{Unit Planning}

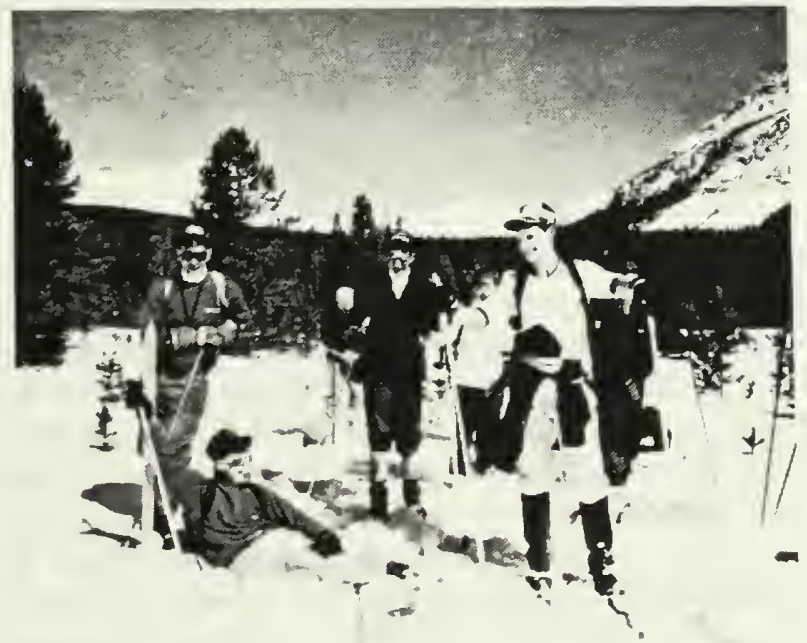


.

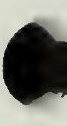

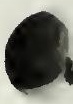




\section{PLANNING A SINGLE-YEAR PROGRAM}

For the single-year course, the content will reflect all six elements of the course, with emphasis on the Foundation level according to student needs. This content will be presented in units that should:

- be thematic

- complement the season

- make effective use of community resources

- be incremental so they build on previously taught knowledge, skills and attitudes

- be consequential so the information is useful to the student in that unit

- be experiential so the student is involved in activities.

Specific guidance in the development of units is provided in sample units that are outlined later in this chapter.

\section{PLANNING A MULTI-YEAR PROGRAM}

The flexibility of the EOE course of studies allows teachers to give emphasis to different levels of learning according to the needs and abilities of the students. Generally, a first-year program in environmental and outdoor education will give most attention to the Foundation level. Where the program is developed over two or three years, the second year might focus more on the Exploration level of the model leading to an emphasis on Empowerment in the final year.

\section{Level I Breakdown}
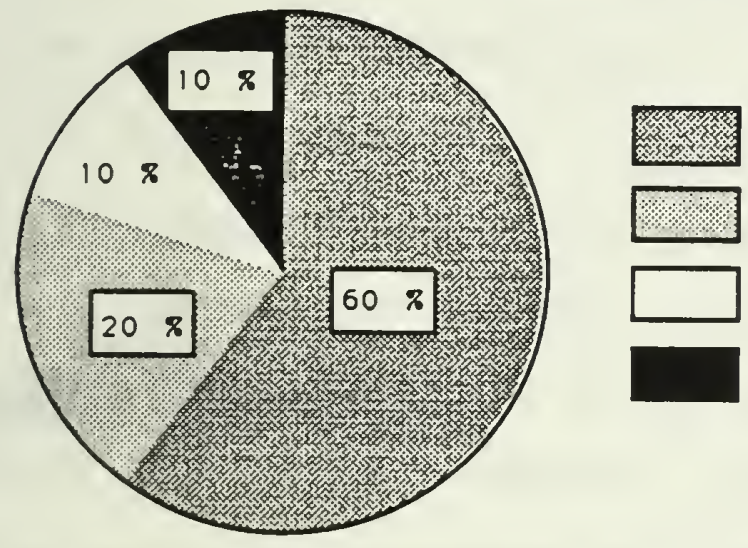

Foundations

$60 \%$

Explorations $20 \%$

Empowerment $10 \%$

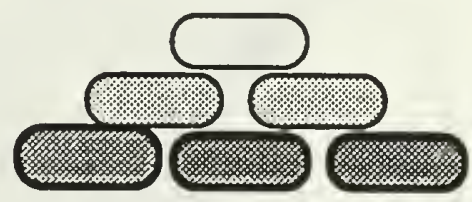

Optional

$10 \%$

In a single-year program, or in the first year of a multi-year program, the development of foundation knowledge, skills and attitudes should be emphasized. The environmental strand of Level 1 provides opportunities for students to study local environments and the general ecological principles that apply to those environments. The personal and group development strand focuses on the personal awareness, communication and group skills that will be needed later in the Exploration level activities. The third component of Level 1 is the development of outdoor skills, in preparation for Outdoor Expeditions. Although a first year environmental and outdoor education course will also include Exploration and Empowerment activities, these will likely receive less emphasis than in years two and three. In year one, the Environmental Investigations component will direct students' attention toward lifestyle issues they can address in their immediate environment (e.g., energy and material use at home and at school). Outdoor Expeditions will involve short trips commensurate with student and teacher abilities. 
Commitment To Action activities will in most instances develop as a follow-up to action plans initiated in Investigations and Expeditions activities.

Level 2 Breakdown
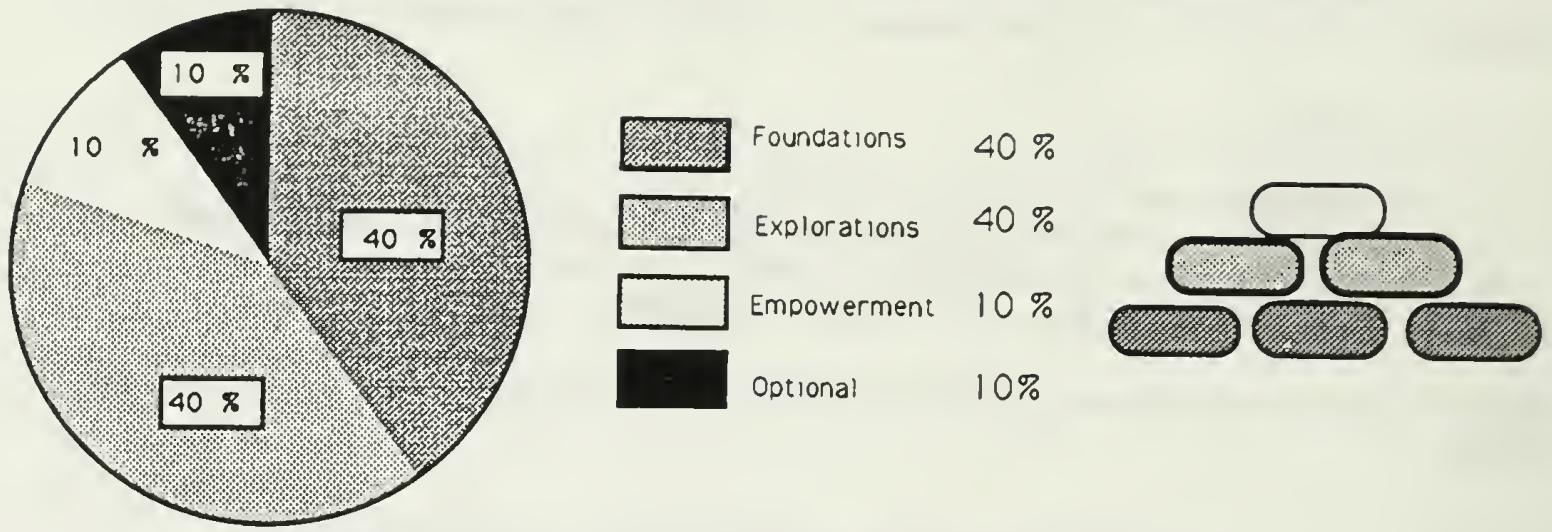

In year two, the emphasis of the course will be on Exploration, but all program elements should be given attention. Elements from the Foundation level should be reviewed and further developed. In the environmental strand students may be provided opportunities to focus on specific issues at a local or regional level. They may review environmental principles that apply to a particular issue before moving into the investigation and problem-solving process. The Commitment To Action element will provide opportunities to follow up on knowledge gained in Environmental Investigations. Similarly, students will have the opportunity to review and develop further theır Foundation knowledge, skills and attitudes, which will allow greater challenge in Outdoor Expeditions. The Commitment to Action in this strand examines ways that students can follow their outdoor activities and trips at school with trips of their own.

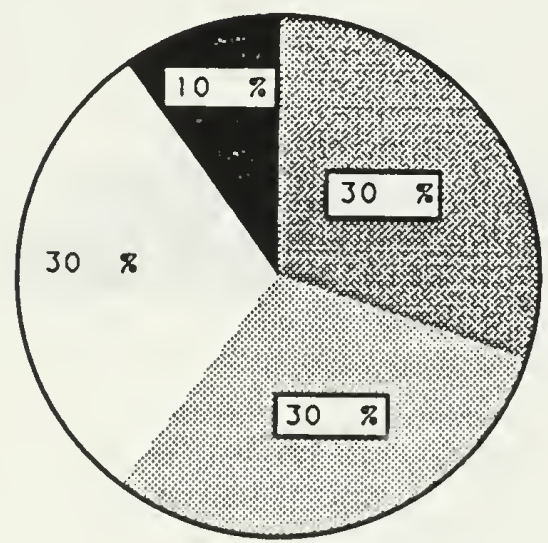

Level 3 Breakdown
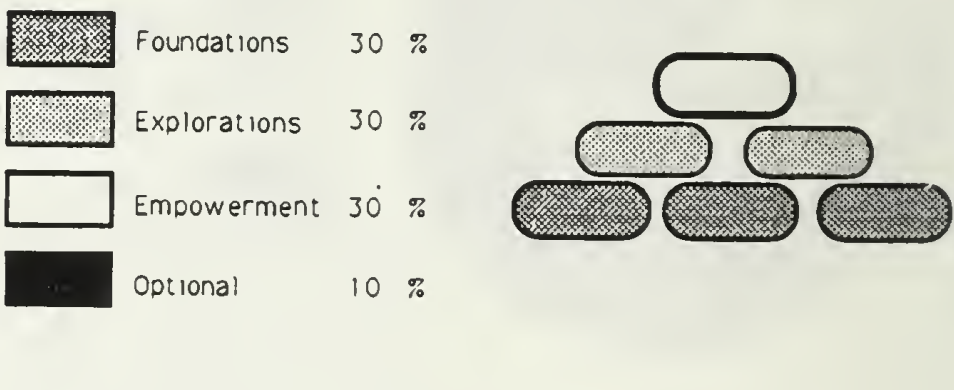

In the third year, the course will focus on the Commitment to Action component. Generally this will be the most self-directed year of the program with students increasingly taking initiative and setting directions for their own learning. The focus of the units will be to provide students with the tools to take effective action in each of the three strands. Effective environmental action is action in context: politically, economically, socially and environmentally. Ecological processes studied in previous years should be reviewed and put into an expanded context. This is generally best accomplished by applying the ecological concepts to an environment not previously studied. In the environmental strand students will have the opportunity to follow a problem-solving model, develop action plans, then develop the skills to implement those actions plans. In the outdoor strand students may be heavily involved in planning and carrying out trips, and thus will develop the skills to do their own trips in the future. 
In the personal and group development strand students will address personal growth issues that will complement their actions in the other two strands, and will plan strategies for enhancing their personal growth beyond the program.

\section{UNIT AND LESSON PLANNING}

The content charts at the end of the Teacher Resource Manual are provided as a tool for planning specific activities in units and lessons. The units and lessons that follow are examples of approaches that might be used in developing the EOE course. They are guidelines only and not meant to dictate how the course is to be taught.

Many of the Foundation knowledge, skills and attitudes are developed over a series of units as information is required. For example, in winter students develop the first aid skills to respond to coldrelated injuries while at the same time developing preventive skills. Similarly, students develop map skills as they assume more and more of the planning and trip leadership responsibilities. This is suggested format and does not preclude delivering a unit focused on first aid or navigation skills.

The following format is provided as a guideline for lesson planning. A blank lesson planner based on this format follows this section.

- Unit

- Element

- Lesson Topic

- General and Specific Learner Expectations

- Materials And Resources Required

- Student Preparation

- Instruction Strategy

- Summary and Follow Through

- Evaluation

YEAR 1/UNIT 1: The Great Outdoors

Sept./Oct.

The purpose of this first sample unit is to lay some groundwork for future units in all three strands of the model:

- For the personal and group development strand the primary intent is to get the students thinking about ways they can be more effective communicators.

- The outdoor strand lays the groundwork for a full day trip including a hot meal on the trail.

- The environmental strand asks the student to identify with one species in the environment they will visit. The students will then identify any impacts that human activities may have on that species. At the Commitment to Action stage, students will look at ways in which they can reduce their impact on the environment.

1. Course Introduction, Expectations and Administration

2. Journal Writing

3. Getting to Know One Another

4. Active Listening 
5. Initiative Tasks

6. Outdoor Clothing

7. Food Planning

8. Fires or Stoves

9. Food Preparation

10. Personal Hygiene

11. Becoming an Expert

12. Interrelationships

13. Wildlife and Me

14. The Great Outdoors

15. Commitment To Action

\section{YEAR 1/UNIT 2: WE'RE BEING AUDITED! Nov./Dec.}

This sample unit starts off with some personal and group development activities followed by a study of how energy flows and materials cycle in the school. The investigative portion of this unit revolves around the interconnectedness of the school with other environments and the question "Can our school have less impact on other environments?" The Commitment to Action element looks at what action students can take to reduce their impact.

\section{LESSONS}

1. Introduction, Expectations and Administration

2. Body language lesson - initiative - Basic Killer game

3. Interpersonal Communication Process

4. Communication skills

5. Initiative tasks

6. Energy flow lesson and game

7. Cycling of materials game and lesson

8. Natural environment

- identify energy flow and cycling of materials

- identify interconnecting web of energy and materials

9. School environment

- identify energy flow and cycling of materials through a school

- self-contained or not

- identify where energy and materials come from and go to

10-11. Student Activities

- Water's Going On?!

- Flip the Switch for Wildlife?

- What Did Your Lunch Cost Wildlife

12. Investigation: Can our school reduce its effect on other environments?

13. Use Environmental Resource Centre materials to conduct school energy audit

14. How is energy in our school being wasted/conserved - energy audit results

15. How to save energy and materials at school

16. Design energy and material saving program

- have students form committee to monitor school energy use over year

17. Commitment to action - reduction of energy and materials

- journal work

\section{YEAR 1/UNIT 3: ARE WE WARM YET?}

Jan. / Feb.

Sample Unit 3 focuses on outdoor skills. Through the unit, a plan is developed that prepares students for a ski trip to a site such as a youth hostel. The first portion of the unit develops winter travel skills and introduces some basic first aid and outdoor preparation skills. The personal and group 
development element addresses values and personal interests, and considers ways these might come into play in the field trip activity. The environmental strand looks at how energy flow and cycling of materials is affected by winter and the adaptations animals and plants must make to winter. Following the expedition students look at the adaptations people make for winter, followed, in turn, by an energy and materials audit for students' homes. The Commitment to Action element looks at how students can reduce their use of energy and materials at home.

\section{LESSONS}

1. Unit Introduction, Expectations and Administration

2. Review of outdoor clothing principles - changes for winter

3. Repair and maintenance lesson, including waxing

4. Skiing lesson

5. Winter hazards

6. Skiing lesson

7. Food planning

- winter requirements

- menu planning

8. First aid simulation design - student design session using information supplied by teacher

9. First aid simulation

10. Ski lesson

11. Map work - schoolyard map exercise

12. Understanding the self-awareness model

- values and their relationship to behaviour

- values are related to beliefs and attitudes

13. Personal interests and activities to pursue on an outdoor trip

14. Researching trip - getting journal ready/trip photographer

15. Winter signs of animals

- adaptations animals make to winter

- how are energy flow and cycling of materials affected by winter?

16. Trip preparation - administration /group and personal gear

- objectives/concerns and expectations

TRIP TO HOSTEL

- journal writing

- animal activities

17. Urban Adaptations

- how do we adapt to winter?

18. Home energy and materials audit

19. Alternatives and consequences to current practices

20. Develop action plan and implement - evaluate?

- check school audit to see if school is having results

21. Commitment to action

- reduce use of energy and materials at home

- plan future outdoor trips - family/club

\section{YEAR 1/UNIT 4: CYCLING}

Mar./Apr.

Sample Unit 4 opens with lessons from the personal and group development element of the program framework, which examines values, communication and interaction within groups. The environmental strand considers cycles of air and gases and man's impact on them. The outdoor strand complements 
the environmental strand by providing the opportunity to consider bicycling as an alternative to fossil fuel vehicles.

The bicycling portion of the unit examines safety, maintenance and repair, and culminates in a half-day trip. The unit ends with a Commitment to Action regarding plans for changes in home, school or community that will improve air quality.

\section{LESSONS}

1. Unit Introduction, Expectations and Administration

2. We All Have Different Values, Beliefs and Attitudes exercise

- Openers Exercise

3. The Interpersonal Gap - communication theory

4. The Pinch Theory

5. Basic Needs of People in Groups exercise

6. Initiative task - TP Shuffle

- Ship Wreck

- Spider Web

7. Interrelationships

- Cycle Factory

- natural systems are interconnected

8. Air cycle

- newspaper article: Our Fragile Future

- general functioning

- how the air cycle can spread effects of localized pollution

9-12. Investigation

- are we having an impact on the air cycle?

- research

- alternatives and consequences

- choices

13. Develop personal action plan

- goal setting

- tree planting in schoolyard?

14. Bicycling - rules of the road

15. Maintenance and repair

16. Hazards - en route organization

17. Emergency response

18. Bicycle first aid simulations

19. Half-day trip in town

20. Review of personal action plan for changes in home, school and community regarding air quality

\section{YEAR 1/UNIT 5: SPRING BREAKUP}

\section{May/June}

Sample Unit 5 begins with a series of lessons from "Dryland Boating, A Resource Manual for Boating Safety Instruction," which prefaces the canoeing lessons that follow later in the unit. In preparation for a half-day trip to a site on the same body of water as the lessons, students are asked to find out about the history of the area, future plans for the site and the wildlife on the site. Students attend a week of canoe lessons with the last lesson extending to a half-day trip to a natural area. The unit then moves on to focus on the water cycle and the role of water in nature and in manmade environments. The Environmental Investigation examines the use of water in the community and means of reducing any negative impacts. 
1. Unit Introduction, Expectations and Administration

2.5. Dryland Boating Package - Red Cross

3. Map reading for trip area - aerial photographs

4. Research trip area - history, wildlife, plans for development

5-10. Week of Canoe Lessons

11. Lake trip on last day of lessons

12-15. Water cycle

- role of water cycle in nature

- water cycle in Alberta

- importance of water cycle in your life

- cycle of water in community

- changes that water can make

- how water moves materials

16. Interviewing skills / Telephone skills

17. Letter writing

18. Visit to water treatment plant

19. Investigation of water cycle through community

- getting information

- guest speaker

- effects of water use

- alternatives and consequences

20. Student personal action

- possible water trips in summer

- journals

\section{YEAR 2/UNIT 1: CAN'T SEE THE FOREST FOR THE TREES Sept./Oct.}

Sample Unit 1 of Year 2 combines outdoor lesson in backpacking with a forestry theme. Many of the outdoor skills are a review of the previous year's studies.

Students are more involved in planning the trip though the teacher has made the necessary bookings in advance and has selected the general area. The environmental strand also incorporates review of basic ecological principles and follows through with an application of those principles to forestry.

Students examine current forestry practices and their consequences, as well as alternatives and their consequences. Students consider the subject in an economic, social and political context as well as an environmental context. Students should also consider their action plans in terms of personal lifestyle changes that they can make, as well as local, regional and global actions that might be taken.

\section{LESSONS}

1. Unit Introduction, Expectations and Administration

2. Review of clothing needs for upcoming overnight backpacking trip and anticipated hazards - i.e. black and grizzly bears

3. First aid scenario - students plan simulation for back-country accident

4. Simulation sessions - debrief

5. Emergency response plan for trip - including helicopter information and victim carries

6. Staying found - what to do when you are lost

7-8. Navigation: map work, including topographic maps and route cards

9. Personal and group hygiene review and minimum impact lesson

10. Students research trip

- determine route with parameters set by teacher 
- visit logged area

11-12. Food - nutrition review

- menu planning for trip

13. Trip preparation including equipment lists

14. Odds and ends for trip - administration (checklists/journals)

- half-day hike with full packs

15. Backpacking trip - overnight - debrief/journal work mini solos

16. Review of basic ecological principles

- energy flow

- cycling of materials

- interrelationships

17-19. Environmental Investigations

- forestry - current practices - guest speakers

- alternatives and their consequences - guest speaker

- student choice and action plans

20. Personal action e.g. planting trees - Forever Trees program

Community action e.g. planting trees

Provincial action e.g. writing letters

YEAR 2/UNIT 2: WE'RE ALL IN THIS TOGETHER NOV./Dec.

Sample Unit 2 combines an outdoor survival theme with an environmental theme focusing on animal survival strategies. The outdoor strand takes students to a survival camp. Animal survival strategies incorporate activities from Operation Lifeline into a series of lessons that look at endangered species.

\section{LESSONS}

1. Unit Introduction, Expectations and Administration

2. Survival exercise - Case Study

3. Navigation - more map work

4. Navigation - introduction of compass - schoolyard setting

5. Review of definition of a group, basic needs of people in groups and awareness of personal needs

6. Preparation for survival camp - equipment check

- journal prep

- solo prep

7. Survival camp with Alberta Fish and Wildife

- play animal game at camp

- solo time

- journal writing

- have officers talk about how animals and plants adapt to seasonal changes

8. Environmental Investigations

- animal survival strategies

9-12. Operation Lifeline materials - four lessons that focus on the questions:

- What is extinction?

- What is habitat?

13. Commitment to action - action to preserve species and habitats

14. Commitment to action - put survival lists together for family vehicles

\section{YEAR 2/UNIT 3: "JACK RABBIT" JOHANNSEN ... HERE WE COME! Jan./Feb.}

Cross-country skiing is the main focus of sample Unit 3. The unit prepares students for winter travel skills such as cross-country skiing and takes students' navigation skills one step further. Students also develop their group skills in the personal and group development strand. The Commitment to Action 
identifies outdoor recreation options and student plans to get involved in some type of winter outdoor recreation activity.

\section{LESSONS}

1. Unit Introduction, Expectations and Administration

2. Review of clothing needs and winter hazards

3. Skill development in cross-country skiing

4. First aid simulation planning

5. First aid simulations

6. Trip research by students

- possible cabins

- what area has to offer

7. Skill development in cross-country skiing

8. Trip logistics

- menu planning

- equipment lists

- administration

9. Navigation - additional compass and map work

10. Navigation - emergency response, evacuation routes, route cards

11. Review of en route organization, role of leaders/group selection of student leaders and groups

12. Skill development in cross-country skiing

13. Understanding people through strengths

14. Task and maintenance functions in groups

15. Conflict resolution

16. Cross-country ski trip to hostel; journals/solo time

17. Commitment to Action - Identifying winter outdoor recreation opportunities and how to access them

YEAR 2/UNIT 4: WIDE OPEN SPACES

Mar. / Apr.

Sample Unit 4 continues the personal and group development process by studying stages of individual and group development. The environmental strand focuses on the prairie ecosystem, beginning with basic awareness and understanding. Following these Environmental Core activities, students will begin an investigation of prairie ecosystems including consideration of current land use practices, alternatives, and the real or potential consequences of each. After a field trip to a prairie grassland area, students will develop personal action plans and work on skills they will need to take effective action.

\section{LESSONS}

1. Unit Introduction, Expectations and Administration

2. Stages of individual development

3. Stages of group development

4. Personal style - bipolar exercise

5. Prairie ecosystem - air/water/soil cycles, energy flow, change

6-11. Environmental Investigations - prairie ecosystem research

- Prairie Conservation Society - guest speaker

- farmland and its effects - soil/water

- alternatives and consequences

12. Effects of human activity on prairies locally/globally

13. Prep for day trip to natural prairie

14. Day trip

15-17. Action

- personal action 
Students should play an active role in planning this sample unit. The unit provides opportunities for students to plan and carry out a river canoe trip. Lake canoeing skills are reviewed and supplemented with basic river canoeing skills for paddling on Class I rivers. Hazards associated with rivers are introduced using films. Students plan the canoe trip with the teacher's help (e.g. paddling groups, food and shelter requirements). As part of their trip preparation students will do a map exercise using the route to be travelled. A local fish and wildlife officer will be brought in to describe the wildlife and signs of wildlife students can expect to see along the route. The students have two environmental options they can choose from. One option examines animal habitat and human impact on those habitats. The second option has more of a science orientation as it examines water quality, in particular the effects of sewage on oxygen content of river water. The final stage involves action planning regarding habitat enhancement or water quality improvement.

\section{LESSONS}

1. Introduction of Unit, expectations and paperwork

2. Review of lake canoeing information - safety equipment/hypothermia

3. Lake canoeing review (on water)

5. River awareness film "Margin For Error"

6. River awareness "Uncalculated Risk" and self-rescue principles

7-9. Introduction to river canoeing (on water)

10. Planning the canoe trip

- paddling and camping groups

- food, shelter and clothing considerations

- packaging considerations

11. Map exercise of route to be travelled

12. Familiarization with wildlife along canoe route (e.g. signs of beaver such as lodges, cuttings, bank slides, scent posts, etc. )

Environmental Option A

13. Animal Habitat - Project Wild Habitat Rummy

14. Animal Habitat - Project Wild On! Deer

15. Project Wild: What Did Your Lunch Cost Wildlife?

Environmental Option B

16. Clean water and its value

17. Introduction of Aquatic Invertebrate Monitoring (AIM) System

18. Test sampling from two sites

19. Canoe trip to McKinnon Flats from Fish Creek Park

20. Evaluation of information gathered on trip

21. Option A - Action Planning: Improving Habitat in the Community (P.W.)

Option B - Water's Going On ?! (P.W.) 


\section{YEAR 3: STARTING POINTS FOR A PLAN}

Unit 1 - Introduce format for year and the framework within which you and your students will plan the year, for example:

- learning expectations

- emphasis on different program elements

- cost considerations

- degree of challenge

- knowledge and skill levels of students

- teacher's right to step in at any time

- students' opportunity to make individual choices

- focus on skills

- identify and develop new skills where needed

- upgrade skills where necessary

- realistic goal setting

- safety

- respect for self, others and environment

- positive approach (always for something rather than against)

Initiation Process

- Have students plan first unit - set up teams - teacher acts as facilitator

- Trips researched

- Investigations - student directed

- Timelines developed 
.

a 

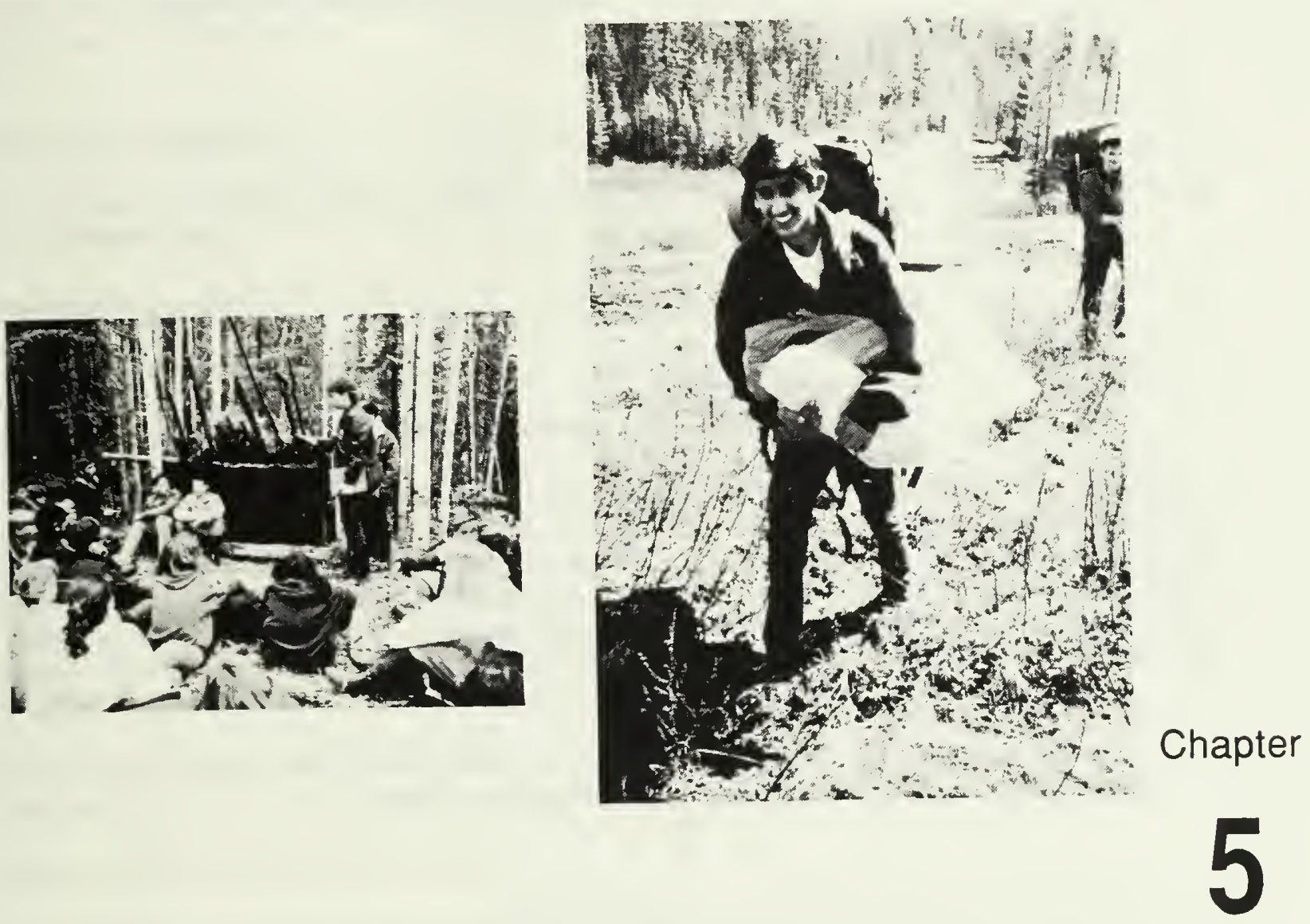

\section{Lesson Planning}

A Model Introductory Unit

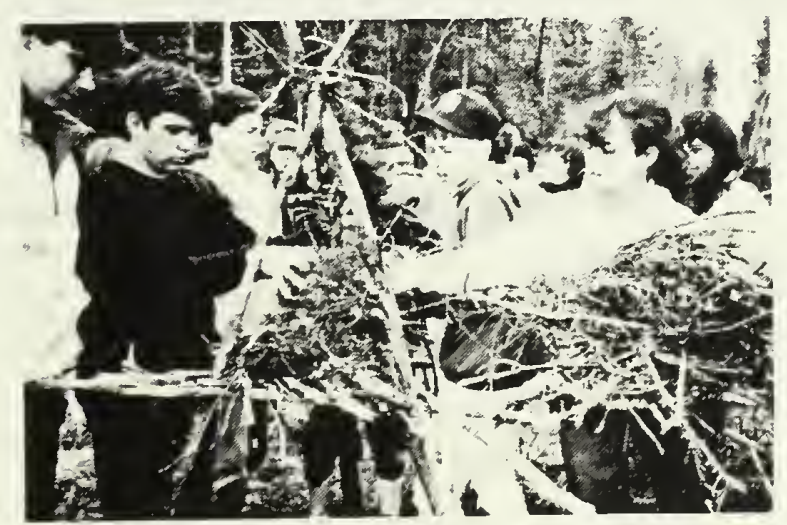




\section{OVERVIEW, UNIT 1.1: THE GREAT OUTDOORS}

Unit Name: The Great Outdoors

Element Name: Personal And Group Development

Lesson Subject: Journal Writing

Lesson: 1

\section{Specific Learner Expectations:}

1. b. Students will recognize that individuals have personal lifestyles and beliefs that help define who they are.

\section{Materials and Resources:}

Journals, pens, blackboard, chalk

\section{Instruction Strategy: Discussions and written work}

1. Explain the function of the journal as a reflective tool for classes and trips. The journal can include written material, poetry, sketches, photographs, sayings, and have bits and pieces of things. The intent is to be as creative as possible. It is the teacher's option to read it or not. You may want to use it as an evaluative tool. Have the students set up the journal in a ringed binder so that they can add a variety of things to it and remove things they don't want you to read. (It is a good idea for teachers to take pictures of students on trips and in class for journals, bulletin boards and parent's nights.)

2. The teacher outlines the course plans for the year and points out where students have an opportunity for input. Have students write down what they expect to get out of the course.

3. Share these expectations with a friend.

4. In groups of four write down the five most important things the group expects to get out of the course. Have first group finished write their expectations on the board.

5. Review these expectations and have the class give you any additions they might have for the list. Discuss the expectations with the class, particularly regarding the realities you face as the course teacher.

\section{Summary and Follow-Through:}

Have the class copy the complete list of expectations, the course outline, costs, equipment required and the mark breakdown into their journals.

\section{Evaluation:}

Formative - Students make entries in journal on expectations for course. 
Unit Name: The Great Outdoors

Element Name: Outdoor Core

Lesson Subject: Introduction and Planning

Lesson: 2

Specific Learner Expectations:

1. d. Students will recognize the role and importance of planning in outdoor activities.

- Students will recognize the role of planning in enhancing safety and enjoyment of outdoor activities.

- Students will recognize the needs and expectations of group members, and all others affected.

- Students will recognize the scope of planning appropriate to different kinds of outdoor activities.

Materials and Resources Required:

Forms required for student and volunteer participation.

\section{Student Preparation:}

Students should be provided copies of required forms and understand their importance as a precursor to student participation.

\section{Instruction Strategy:}

Present information about unit and student involvement in planning it.

Have question and answer period followed by a brainstorming session in small groups.

\section{Summary and Follow-Through:}

Have students rank the outcome of their small group sessions and present their priorities to the class. Help the class establish priorities for the unit.

\section{Evaluation:}

Formative - Student participation.

\section{Unit Name: The Great Outdoors}

Element Name: Personal and Group Development

Lesson Subject: Getting to Know One Another

Lesson: 3

\section{Specific Learner Expectations:}

3. a. Students will demonstrate skills in communicating intents and ideas both verbally and nonverbally.

b. Students will recognize the effects of their communications and actions on others.

- Students will recognize and accept that the reactions of others may reflect a different perspective than one's own.

- Students will demonstrate sensitivity to others. 
Materials and Resources Required:

1 ball/student

\section{Student Preparation:}

Appropriate for students who are not familiar with one another's names. Some students will take this as an opportunity to demonstrate their ability to throw a ball very hard. Caution students that only softly thrown underhand tosses are appropriate.

\section{Instruction Strategy:}

Activity 1 Have the groups form a circle and have each student repeat his or her name to the groups. Explain that students will be passing the ball back and forth across the circle. Before the student throws the ball to someone he or she must call the person's name and establish eye contact. After the group has practised this activity rearrange the students' positions in the circle. Practise this activity again. Now add one or two more balls to the passing game. To further test the students' recall have them thank the person who just passed the ball to them while calling out the name of the person they are passing the ball to.

Activity 2 Introduce initiative tasks. Divide the class into groups of seven or eight. Explain to them that keeping the ball in the air requires the co-operative efforts of all the entire group. Have the group pass the ball around the circle so that every student in the circle gets a chance to catch and pass the ball. Do not allow any one student to get the ball more than once. Have the students repeat the sequence until they can follow the sequence consistently. Add balls to the circle one at a time. The initiative task is to see how many balls the group can incorporate into the passing game. Have the students identify what they must do as a group to meet the challenge.

Start your description of the Environmental and Outdoor Education program by explaining that the success of the course will be partly dependent on how well the group works together over the year. Working together involves communication and cooperation similar to that used in the initiative task.

\section{Summary and Follow-Through:}

Ask the groups if anyone would like to try to name all of the students in the group. Do people like to be called by their name? Why?

\section{Evaluation:}

Formative - Have students write all the names of the students in the class in their journals.

Unit Name: The Great Outdoors

Element Name: Personal And Group Development

Lesson Subject: Active Listening

Lesson: 4

\section{Specific Learner Expectations:}

3. a. Students will demonstrate skills in communicating intents and ideas both verbally and nonverbally. 
Materials and Resources Required:

Rules for brainstorming, blackboard or overhead

Communication Skills I - booklet from the Recreation Parks and Wildlife Foundation

\section{Student Preparation:}

1. Teacher lists rules for brainstorming.

2. Teacher poses question: "What are some of the communication skills a ship's crew must have to function effectively?" Post this list on the blackboard. "Getting along" should include listening to one another.

\section{Instruction Strategy:}

1. Teacher introduces active listening. To the whole group, pose the question: "What does active listening mean?" Tell the group to write their response in their journals. Ask for responses. You should be looking for a definition of active listening, which is something like participating and applying oneself to hearing what another person is saying.

2. The following questions may help your students focus.

- Are students attentive and following the conversation or are they daydreaming?

- Are they using each other's names?

- Does their body language show that they are attentive?

- Can they think of other ways that they can show that they are attentive?

- Do the students establish eye contact with one another? Is eye contact appropriate in all cultures?

\section{Exercises}

a. Have students form groups of three. Designate $A, B$ and $C$ roles within each group.

Person $A$ is the observer and giver of feedback.

Person $B$ is the active listener.

Person $C$ is the talker.

Person $C$ is to talk to $B$ on one of the topics listed below.

King Arthur's Environmental Round Table

Friendly Aliens have just landed. What would you ask them?

Person $\mathrm{C}$ will take two or three minutes to explain. Use one topic from this list for your first exercise and the remainder in following exercises.

At the end of that time, person $A$, the observer, will give feedback to person $B$, the active listener, on how they were using active listening skills.

Once the entire class is finished, switch roles and repeat until all three people have had a chance to be the observer, the active listener and the talker. Follow this up with a discussion on the active listening skills that the students were using.

With the students still in their groups, have them switch roles again and have the observers exhibit one form of poor active listening. After each session have the group discuss the effect of the poor listening on the speaker.

Tell the class that as soon as one session is done, switch roles and repeat until all three people have had a chance to be the observer, the active listener and the talker. 
Student Summary and Follow-Up:

Have students give examples of where they could use active listening.

\section{Evaluation:}

Formative - Students describe in their journal how they consciously used active listening principles at least once after the active listening exercise in class.

\section{Unit Name: The Great Outdoors}

Element Name: Personal and Group Development

Lesson Subject: Initiative Tasks

Lesson: 5

\section{Specific Learner Expectations:}

5. Students will demonstrate an understanding of group process and develop skills in group process.

a. Students will demonstrate awareness and responsibility for their actions as a members of a group.

Materials and Resources Required: (Silver Bullets)

$\begin{array}{ll}\text { Electric Fence } & \text { Spider's Web } \\ 1 \text { rope }\left(30^{\prime}\right) & \text { Nylon cord } \\ 2 \text { posts ( goal posts or } 2 \text { trees) } & 2 \text { trees close together } \\ \text { Helmets } & \text { A small bell }- \text { Spider Dinner Bell } \\ 1-4^{\prime} \times 4^{\prime} \text { beam } & \end{array}$

Student Preparation:

Stress the importance of spotting. Stress that students can't run at the fence and that they must be in contact with someone at all times. Have the students identify how groups work together effectively.

\section{Instruction Strategy:}

Use goal posts on the playing field to set up the electric fence. If helmets are available students should wear them. Use the same guidelines for the Spider's Web and try to keep the two initiative sites out of sight from one another so that students cannot see solutions to initiatives they haven't tried yet.

\section{Summary and Follow-Through:}

Questions for the group: How did your group work together? How could your group work together more effectively?

\section{Evaluation:}

Formative - Participation, Journal Entry 
Unit Name: The Great Outdoors

Element Name: Outdoor Core

Lesson Subject: Outdoor Clothing

Lesson: 6

\section{Specific Learner Expectations:}

3. a. Students will select personal and group gear for outdoor activities.

- Students will select personal equipment for outdoor expeditions with particular consideration to maintaining body temperature and protection from injury.

\section{Materials and Resources Required:}

Clothing you would bring on a trip

Survival exercise that you make up

Film on hypothermia

CBE Personal Equipment

\section{Student Preparation:}

Explain that spaceship crews must often wear specialized gear as they move around their ship or outside their ship to protect themselves from the cold or intense sunlight. When moving in some areas of our ship we must also bring along specialized equipment to make the environment more hospitable. Discuss parallels on earth.

\section{Instruction Strategy:}

1. Wear all the clothing you would take on an outing. Discuss how your body gains heat and how it loses heat. As you take off each item explain why you would take that item. Suggest other types of clothing and materials that would be good substitutes. This should help students when searching for clothing for their outdoor wardrobe. In your presentation include information on the layering principle.

2. Follow up the clothing exercise with a survival situation in which students (in small groups) must decide on 10 items they would like to have in that situation. Have them rank the list by priority and give their reasons to the class. Examples of survival situations include: lost while hunting, snowbound in your vehicle or a snowmobile breakdown in a remote area.

\section{Summary and Follow-Through:}

Emphasize the importance that proper gear selection plays in being prepared for and avoiding emergencies. Have students decide their personal gear inventory for the upcoming trip. If you have time you could talk about how other passengers on The Great Outdoors adapt to changing conditions (e.g. migration, hibernation, changing fur colour, winter fat, leaf loss, etc.)

\section{Evaluation:}

Formative - Students will participate in the survival exercise. 
Unit Name: The Great Outdoors

Element Name: Outdoor Core

Lesson Subject: Food Planning

Lesson: 7

Specific Learner Expectations:

3. b. Students will plan to meet food needs during extended outdoor activities by demonstrating an understanding of:

- Nutrition requirements.

- Portability and preservation.

- Food preparation techniques (e.g., cooking, fires and stoves).

Materials and Resources Required:

Outdoor Cookbooks

Favourite recipes

C.B.E. Nutrition Booklet

Menu Planner

Student Preparation:

Discuss the value of thorough food planning on trips (i.e. morale, energy).

\section{Instruction Strategy:}

Discuss what would constitute a good menu (including quantities). If possible, arrange for a guest presentation by a health nurse or home economist. Once the students have the basics, have them:

1. Divide into cooking groups and plan the menu for the upcoming trip including how to package the food.

2. Draw up a menu and assign responsibility for each meal. If your first trip is a day trip have them prepare a hot meal on the trip.

3. Decide which meal they will prepare for their schoolyard cookout. If your first trip is a day trip have the students cook a different meal for their schoolyard cookout.

4. Decide when they will prepare the food for the trip.

5. Discuss tips that will make outdoor cooking easier.

\section{Summary and Follow-Through:}

Emphasize the responsibility students have to the group to have their particular meals ready. You may want to have this lesson extend for another period.

The class could also discuss animal and plant nutrition and the adaptations that are made (e.g. generalists such as wolves and black bears, or specialists such as koala bears and humming birds).

Evaluation:

Formative - Students will submit a group menu plan. 
Unit Name: The Great Outdoors

Element Name: Outdoor Core

Lesson Subject: Fires and Stoves

Lesson: 8

Specific Learner Expectations:

3. b. Students will plan to meet food needs during extended outdoor activities by demonstrating an understanding of:

- Nutrition requirements.

- Portability and preservation.

- Food preparation techniques (e.g., cooking, fires and stoves).

Materials and Resources Required:

$\begin{array}{ll}\text { Stoves } & \text { Fuel } \\ \text { Pots } & \text { Soap } \\ \text { Grill } & \text { Fire extinguisher } \\ \text { Water bottles } & \text { First aid kit }\end{array}$

If you are using fires, get permission to establish fire pits on school property or teach the students to build a fire without leaving a fire scar.

Instruction Strategy:

Use the booklet C.B.E. Fires And Stoves, pp. 5-29

Summary and Follow-Through:

Review the responsibilities that students have when using fires and stoves.

Evaluation:

Formative - Students will learn to use fires or stoves safely and with minimum impact.

Unit Name: The Great Outdoors

Element Name: Outdoor Core

Lesson Subject: Food Preparation

Lesson: 9

Specific Learner Expectations:

3. b. Students will plan to meet food needs during extended outdoor activities by demonstrating an understanding of:

- Nutrition requirements.

- Portability and preservation.

- Food preparation techniques (e.g., cooking, fires and stoves).

Materials and Resources Required:

Stoves

Pots

Grill

Water bottles
Fuel

Soap

Fire extinguisher

First aid kit 


\section{Student Preparation:}

After students have planned a menu for their trip have them bring in the ingredients for one meal and have a schoolyard cookout.

\section{Instruction Strategy:}

Have your students cook their meal after you do a demonstration outlining safety concerns, including how to fuel the stove. Have students move into their food groups and have them prepare a meal. Encourage them to set up a smorgasbord so they can taste a variety of meals.

If you are using fires, discuss the basic principles of fire building and environmental considerations when building fires.

\section{Summary and Follow-Through:}

Discuss the responsibilities one assumes when using stoves or fires.

\section{Evaluation:}

Formative - Students experience what it is like to cook outside. Students taste a variety of meals prepared in the outdoors.

Summative - Students participate in food preparation and cooking.

\section{Unlt Name: The Great Outdoors}

Element Name: Outdoor Core

Lesson Subject: Personal Hygiene

Lesson: 10

\section{Specific Learner Expectations:}

3. c. Students will demonstrate knowledge of the techniques required for maintaining hygiene in outdoor settings in the following areas:

- Water purification.

- Personal cleanliness.

- Group hygiene.

\section{Materials and Resources Required:}

Water purification tablets

Water bottles

C.B.E. Trip Planning, pp. 67-68

\section{Student Preparation:}

Exploring the outdoors involves taking care of the environment as well as ourselves.

\section{Instruction Strategy:}

1. Brainstorming session on sources of pollutants in the outdoors. What can you do to protect yourself from them? 
2. Consider ways to manage personal waste in the outdoors:

- Where and how to build latrines and how to use them.

- What to do with toilet paper.

- Seasonal considerations.

- Where to locate sump holes and what goes into them.

3. Use of soap - types, where to wash.

4. Garbage - pack in as little as possible, burn only paper, not plastic or tin foil, burn cans to remove smell and pack them out (assuming that you are cooking over a fire), storage of garbage overnight.

5. Establish a policy concerning what students will do with garbage left by others.

6. Try drinking water purified with water purification tablets.

7. How can you pack your food to minimize waste?

\section{Summary and Follow-Through:}

What contributes to water pollution? What can't be treated with water purification tablets? Is clean water important to you?

How is waste management handled in nature (e.g. bird feces sacs, hare pellets, territory markers, gut bacteria)?

Unit Name: The Great Outdoors

Element Name: Environmental Core

Lesson Subject: Becoming an Expert

Lesson: 11

\section{Specific Learner Expectations:}

1. a. Students will demonstrate an awareness of local and global environments by developing:

- Skill in observing and describing an environment based on first-hand observations.

- Knowledge of some distinguishing features of local and global environments.

- Knowledge of tre diversity of life found in these environments.

\section{Materials and Resources Required:}

Library books - field guides to animals

Animal tracks

Hunter education books

RPWF poster series

\section{Instruction Strategy:}

1. Have students select from a list you provide an animal from the environment the class will be travelling to.

2. Working in pairs, have students make up a character profile on their animal using the resources provided.

3. Have the students make entries in their journal that will help them identify the species from signs in the field. Diagrams, pictures and notes can also be included.

4. Have students identify organisms that are interconnected with their organism to meet needs for shelter or food. 


\section{Summary and Follow-Through:}

Discuss with students their first-hand observations of their environment.

\section{Evaluation:}

Formative - Students participate in research exercise about their animal species and work effectively in pairs.

Summative - Students make journal entries about their species. Journals will be one of the student products considered in evaluation of the unit.

Unit Name: The Great Outdoors

Element Name: Environmental Core

Lesson Subject: Interrelationships

Lesson: 12

Specific Learner Expectations:

2. a. Students will demonstrate skill in identifying interactions and ongoing changes in an environment.

\section{Materials and Resources Required:}

Make up animal game (e.g., Calgary Board of Education: Animal Game) using recycled plastic of different colours and shower curtain rings to make tags.

Use sport pinnies for different groups (e.g. herbivores and carnivores).

\section{Student Preparation:}

Safety goggles if possible and concern for each other while playing the game.

Instruction Strategy:

1. When possible, have students assume roles of the species they have chosen to study.

2. Have the students help you construct the game, e.g. cut out tags etc.

3. Explain game, rules and safety using Animal Game guidelines.

Possible modifications - rather than introducing hunter at the end of the game have the hunter involved in the game with specific quotas on kills and only certain animals that can be shot. Rather than having hunters able to kill on sight, require them to throw something such as old sock stuffed with other socks or rags.

\section{Summary and Follow-Through:}

Discuss game with students. Discuss how they felt as their particular animal. Did the game illustrate the idea of interconnectedness?

Evaluation:

Formative - Have students enter their animal's perspective on the animal game in their journals. 
Unit Name: The Great Outdoors

Element Name: Environmental Investigations

Lesson Subject: Wildlife and Me

Lesson: 13

\section{Specific Learner Expectations:}

1. Students will demonstrate skill in environmental investigations.

\section{Materials and Resources:}

Local Fish and Wildlife officer/other knowledgeable member of community (possibly someone with different views).

\section{Student Preparation:}

Discuss questions students could ask. Reminder - Always be for something rather than against thing.

\section{Instruction Strategy:}

1. In a presentation of up to 10 minutes per student, have presenter discuss what's being done and the results. Ensure the action involves the students in a significant way.

2. Factors affecting area

3. Special problems unique to this area

4. Student Questions (i.e. What can students do to affect wildlife positively? Questions about specific interrelationships).

\section{Summary and Follow-Through:}

Have the students determine what they feel would be the best plan of action they could take to minimize their impact on their particular species.

\section{Evaluation:}

Formative - In journals students will write a 200-word article on best introducing their species, and how it is affected by man. Students should recommend the best plan of action they can take to reduce their impact on their chosen species, with some suggested alternatives.

\section{Unit Name: The Great Outdoors}

\section{Element Name: Outdoor Core}

Lesson Subject: Into the Void

Lesson: 14

\section{Specific Leaner Expectations:}

2. Students will develop competence in trip preparation and safety.

\section{Student Preparation:}

Have students identify where they can collect the gear they will need for the trip. 
Materials and Resources Required:

Group and personal equipment lists

Examples of the equipment needed

Instruction Strategy:

1. Teachers should pick a trip commensurate with their abilities to lead as well as the students' abilities to follow.

2. The trip should involve some degree of preparation so that the students make use of the outdoor core work they have been doing.

Summary and Follow-Through:

Conduct a gear check before the trip.

\section{Evaluation:}

Evaluate whether students are prepared.

Unit Name: The Great Outdoors

Element Name: Commitment To Action

Lesson Subject: Let the Bells Ring Out

Lesson: 15

Specific Learner Expectations:

3. d. Students will identify and act on opportunities in their communities to take actions that may lead to positive impacts on local, regional, national and global environments.

Materials and Resources Required:

Rules for brainstorming

Addresses for MPs and MLAs

Addresses of local newspapers

\section{Student Preparation:}

Have students brainstorm ideas about how the concept of The Great Outdoors can be conveyed to others.

\section{Instruction Strategy:}

1. Review goal-setting strategies so that students can set realistic projects for themse'ves.

2. The projects should be relatively short.

\section{Evaluation:}

Summative - Evaluate the student's project. 


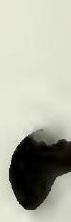




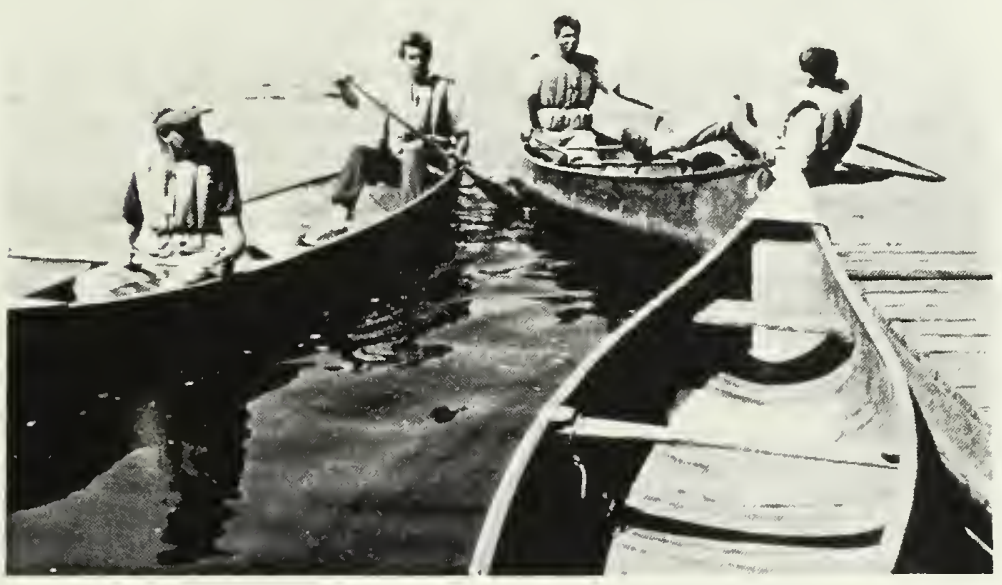

Chapter

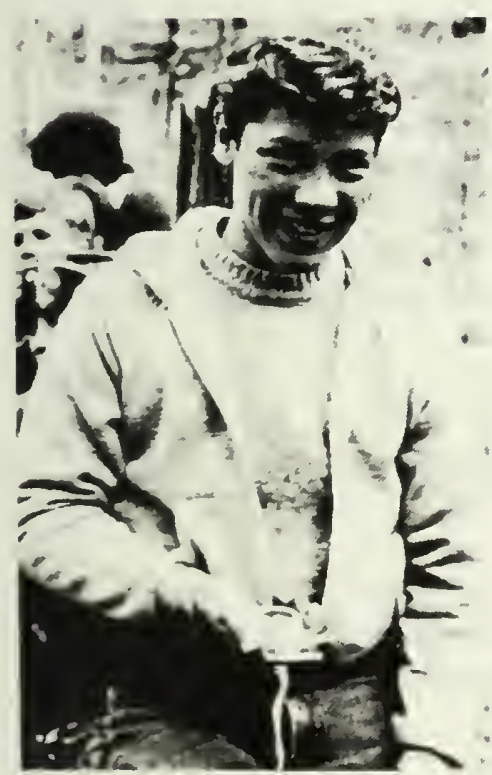

\section{Lesson Planning}

A Model Intermediate to Advanced Level Unit

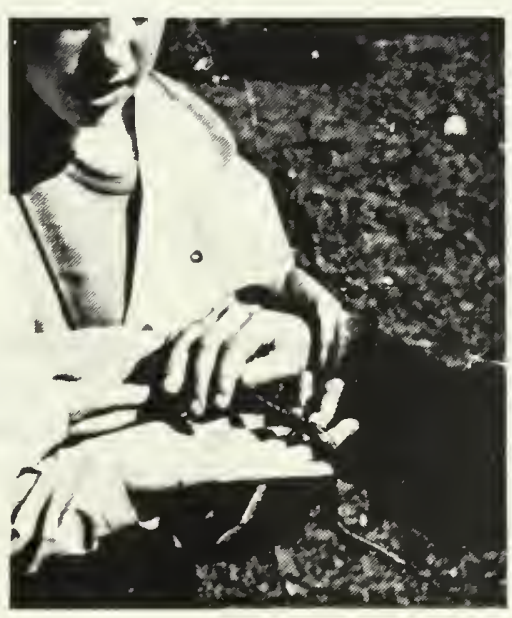




\section{OVERVIEW, UNIT 2.5: RIVER TRIPPING}

Unit Name: River Tripping

Element Name: Outdoor Core

Lesson Subject: Introduction of Unit, Learning Opportunities and Responsibilities

Lesson: 1

Specific Learner Expectations:

1. d. Students will recognize the role and importance of planning in outdoor activities.

- Students will recognize the role of planning in enhancing safety and enjoyment of outdoor activities.

- Students will recognize the needs and expectations of group members, and all others affected.

- Students will recognize the scope of planning appropriate to different kinds of outdoor activities.

Materials and Resources Required:

Forms required for student and volunteer participation

Pictures or slides from previous trips

\section{Student Preparation:}

Importance of forms as a precursor to student participation.

\section{Instruction Strategy:}

Present information about unit and student involvement in planning it.

Have question and answer period followed by a brainstorming session in small groups.

\section{Summary and Follow-Through:}

Have students rank the outcome of their small group sessions and present their priorities to the class. Help the class establish priorities for the unit.

\section{Evaluation:}

Formative - Student participation.

\section{Unit Name: River Tripping}

Element Name: Outdoor Core

Lesson Subject: Unit Planning

Lesson: 2

\section{Specific Learner Expectations:}

2. Students will develop competence in trip preparation and safety.

a. Students will develop the knowledge, skills and attitudes necessary to prepare for outdoor expeditions in ways that will ensure the safety and well-being of those involved.

b. Students will be aware of, list and take the steps necessary to anticipate emergencies and survival situations. 
Materials and Resources Required:

Information on trips available in the area.

Possible resource person familiar with the group's skills and the potential trips in the area.

\section{Student Preparation:}

The students must understand the parameters that planning must follow and that the teacher has the power of veto.

Instruction Strategy:

Complete the unit planning with students and have students assign individual tasks for planning the trip.

\section{Summary and Follow-Through:}

Assign individual student tasks with completion schedules.

\section{Evaluation:}

Summative - Students will accomplish the tasks assigned.

Unit Name River: Tripping

Element Name: Outdoor Core

Lesson Subject: Review of Lake Canoeing Safety

Lesson: 3

\section{Specific Learner Expectations:}

2. a. Students will develop the knowledge, skills and attitudes necessary to prepare for outdoor expeditions in ways that will ensure the safety and well being of those involved.

Materials and Resources Required:

Red Cross Basic Boating Safety Package

Slide projector

Equipment handout

Hypothermia handout

Bucket and ice

\section{Student Preparation:}

Have students review information from previous instruction in lake canoeing and canoeing safety they may have had.

\section{Instruction Strategy:}

Review the hazards associated with cold water.

Have students time how long it takes them to tie up their shoelaces. Then have them soak their hands in a bucket of ice water for a minute. Time the same students as they tie their shoelaces up again. Discuss the effects of cold water and the progressive stages of hypothermia. 
Present the students with the Equipment Handout and have them decide which items they will take with them on their trip.

Review the safety items which the Department of Transport (DOT) requires vessels under 5.5 meters to have on board. Also review the recommended list of safety items which small craft should have.

\section{Summary and Follow-Through:}

Students should identify items on the DOT lists which they have at home and if not, where they can get these items.

\section{Evaluation:}

Summative - Students will arrive to paddle with all the items required or recommended by the DOT.

\section{Unit Name: River Tripping \\ Element Name: Outdoor Core \\ Lesson Subject: Lake Canoeing Skills Review \\ Lesson: 4,5}

Specific Learner Expectations:

5. a. Students will develop skill in a variety of outdoor activities and modes of travel.

\section{Materials and Resources Required:}

Services of canoe instructors and equipment (e.g. canoes, paddles, bailers, whistles, PFDs)

Transportation to site

\section{Student Preparation:}

Review basic parts of canoe and paddles.

\section{Instruction Strategy:}

Have instructors review the basic lake paddling skills with the students and their application of these skills to rivers.

\section{Summary and Follow-Through:}

Have students practise strokes such as the river "J," "J" stroke and the low brace, so that they can paddle their canoes in a straight line on the river and right the canoe if it tips.

\section{Evaluation:}

Summative - Evaluate student canoeing performance on final day of trip. All students should have a chance to stern their canoe on the last day. 
Unit Name: River Tripping

Element Name: Outdoor Core

Lesson Subject: River Awareness (Session 1)

Lesson: 6

Specific Learner Expectations:

5. a. Students will develop the skills to recognize and assess physical hazards imposed by particular terrain hazards and conditions.

Materials and Resources Required:

River Awareness package by the Alberta Recreational Canoeing Association

Video/ Film: "Margin for Error" (22 min.)

VHS video playback unit $16 \mathrm{~mm}$ film projector and screen

Flip chart stand/paper/markers or blackboard/chalk

Handouts - equipment checklists from River Awareness package

\section{Student Preparation:}

Before showing the film ask the students to keep the following questions in mind:

What are some of the things students should consider taking along on an overnight trip?

What are some of the ways to decide if a section of river is within the ability of your group?

What problems could you expect to face on your trip?

\section{Instruction Strategy:}

Show the film and have the students respond to the questions in small groups.

Have each group present their answers to the group.

Handout Equipment Checklists.

\section{Summary and Follow-Through:}

Explain that the degree of difficulty of the river shown in the film is much more difficult than the river they will be paddling. However, the planning principles will be same. The degree of difficulty of the river in the film necessitates helmets and extra flotation. These items are not necessary for the Class I rivers the class will be paddling.

\section{Evaluation:}

Formative - Students will participate in group discussions that address the questions above.

Unit Name: River Tripping

Element Name: Outdoor Core

Lesson Subject: River Awareness (Session 2)

Lesson: 7

\section{Specific Learner Expectations:}

5. a. Students will develop the skills to assess hazards and the danger they represent to the wellbeing of individuals and the group. Students will develop an understanding of the precautions necessary to avoid or respond to these hazards. 
Materials and Resources Required:

Film/video - "Uncalculated Risk" (15 min.)

$16 \mathrm{~mm}$ film projector $\mathrm{VHS}$ playback unit

Screen

Blackboard and chalk, or flip chart/paper/felt marking pens

River Hazards handout

\section{Student Preparation:}

Introduce "Uncalculated Risk" by telling the group the film shows a number of river hazards that might be encountered by paddlers. As people watch the film, have them identify and record each hazard they see. Mention that some of the river features are not found on Class I rivers, but that they must record all hazards.

\section{Instruction Strategy:}

Have the students identify:

a. River hazards (features found on rivers)

b. Equipment hazards (problems that arise as a result of equipment failure or loss)

c. Tactical hazards (errors in paddler judgment that can lead to injury or death).

Remind students that some of the river features in the film are found on Class II rivers or greater. Finish the exercise by handing out the information on river hazards.

\section{Summary and Follow-Through:}

Have students read the River Hazards handout and include this information in their journals.

\section{Evaluation:}

Summative - Students will behave in a safe manner on the river trip (i.e. wearing their life jackets correctly, staying behind the lead boat, not splashing on cold rainy days).

\section{Unit Name: River Tripping}

Element Name: Outdoor Core

\section{Lesson Subject: Introduction to River Canoeing}

Lesson: $8,9,10$

\section{Specific Learner Expectations:}

5. a. Students will develop skill in a variety of outdoor activities and modes of travel.

\section{Materials and Resources Required:}

Local canoeing club for equipment and river canoeing expertise

Transportation to site

Change of clothing for each student

Rain gear when necessary

Wet suits if available 
Student Preparation:

Previous two lessons.

\section{Instruction Strategy:}

Find an appropriate river teaching site (e.g. large eddies, no downstream hazards). Have a vehicle on site or have access to a nearby building to warm students up if necessary. A thermos of warm, sweet fluids or a large fire may be other options worth considering in the event of a capsize.

\section{Summary and Follow-Through:}

Review the possibilities of the canoe capsizing and the rescue procedures that must follow. Emphasize the importance of waterproofing equipment, particularly clothing and sleeping bags.

\section{Evaluation:}

Summative - Students will attend the river paddling sessions with the required gear, including spare clothing.

Unit Name: River Tripping

Element Name: Outdoor Core

Lesson Subject: Map Skills

Lesson: 11

\section{Specific Learner Expectations:}

4. b. Students will develop the skills necessary to interpret and apply route information while en route, including map reading skills.

\section{Materials and Resources Required:}

Topographic maps of trip

Slides of trip

Slide projector/screen

\section{Student Preparation:}

Explain to students that they will be studying maps of the area they will be canoeing in. Have them look for landmarks that will help them estimate distances while they are on the river.

\section{Instruction Strategy:}

Show students a slide show using slides from a previous trip (which focuses on the topography of the route). Have students work in small groups with each group having a set of maps to follow during the slide show. Have the groups identify the access and egress points and landmarks (e.g., bridges, power lines, islands, steep banks, incoming rivers). Explain to student how distances can be measured between points on a river.

\section{Summary and Follow-Through:}

Explain to students that you will not be answering any questions about distances while on the river. Students will be expected to recognize landmarks and estimate distances themselves. 


\section{Evaluation:}

Formative - Have each student identify at least two landmarks on the map and enter these landmarks in their journals along with the estimated distance to each landmark from the access point.

\section{Unit Name: River Tripping}

Element Name: Environmental Core

Lesson Subject: Wildlife Awareness

Lesson: 12

\section{Specific Learner Expectations:}

1. a. Students will demonstrate an awareness of local and global environments by developing:

- Skill in observing and describing an environment based on first-hand observations

- Knowledge of some distinguishing features of local and global environments

- Knowledge of the diversity of life found in these environments.

\section{Materials and Resources Required:}

Invite your local Fish and Wildlife officer or amateur naturalist to make a presentation to the class on the birds and animals (and their signs) they are likely to see during their trip. Encourage speakers to use slides and other props to make it more interesting for the students (e.g. beaver cuttings).

Bird and animal guides, Hinterland Who's Who series.

\section{Student Preparation:}

Ask students to identify animals and evidence of their presence in an area that they are familiar with. Have students identify one animal that they will become an expert on.

\section{Instruction Strategy:}

Introduce the guest speaker.

\section{Summary and Follow-Through:}

Have the students prepare a list of 10 characteristics that will help them identify their animal or signs of their animal.

\section{Evaluation:}

Formative - Have students enter information on their particular animal in their journals. 
Unit Name: River Tripplng

Element Name: Environmental Investigations

Lesson Subject: Clean Water and Its Value

Lesson: 13

Specific Learner Expectations:

1. a. Students will demonstrate initiative and insight in identifying questions for investigation.

\section{Materials and Resources Required:}

a. One large clear container that will hold three litres of water, three graduated cylinders and overhead of the water cycle (posters of the ocean, glaciers or ice cap if possible).

b. A clear flat-bottomed pan or box, four or five sponges, a watering can, four clear jars, red or blue food colouring, a meat baster and two quarts of sand.

Living Lightly on the Planet, pp. 56-64.

\section{Student Preparation:}

Students will study the distribution of the earth's water. Have any students ever experienced a water shortage or had to treat their drinking water? What was it like?

\section{Instruction Strategy:}

Follow Investigations 1 and 2 in Living Lightly on the Planet.

\section{Summary and Follow-Through:}

Have students identify where the water they use comes from and where it goes.

\section{Evaluation:}

Summative - Students will demonstrate an understanding of the fresh water available on the planet and the effects of humans on it.

\section{Unit Name: River Tripping}

Element Name: Environmental Investigations

Lesson Subject: Aquatic Invertebrate Monitoring

Lesson: 14

\section{Specific Learner Expectations:}

1. c. Students will demonstrate skill in researching questions, problems and issues.

Materials and Resources Required:

AIM kit - FEESA

AlM poster

A sample of aquatic invertebrates from local stream or river channel (two people can collect a kick sample with a pair of old pantyhose stretched around a coat hanger). Collect two samples from above and below a water treatment plant. Save the downstream sample for the following class. 
Collect the sample at least a half-kilometre below the outlet. A sewage treatment plant will likely give excellent results as the assimilation of the organic portion of the sewage by the microorganisms in the river will cause the oxygen levels of the water to drop. Hip waders and life jackets are useful when collecting samples.

Petri dishes

Magnifying glasses

\section{Student Preparation:}

1. What do aquatic animals need to survive?

2. Why would the animals that live in the river be a good indicator of the quality of the water?

\section{Instruction Strategy:}

1. Show diagrams of the five invertebrates (see overhead).

2. Explain that the bugs have varying needs when it comes to oxygen.

3. Divide the class into five groups and have each group name one invertebrate. Explain that each bug should have a name that reflects its appearance.

4. Each group is to come up with a name and the reasons for that name as well as some theories about how it manages to stay in one place on the river bottom (Theory of Attachment). Students should have live specimens to examine.

\section{Summary and Follow-Through:}

Each group presents their bug name and Theory of Attachment to the class.

Evaluation:

Formative - Students will demonstrate an understanding of how invertebrate monitoring can be used as an indicator of pollution levels in rivers.

Unit Name: River Tripping

Element Name: Environmental Investigations

Lesson Subject: Aquatic Invertebrate Monitoring - Interpreting the Results

Lesson: 15

\section{Specific Learner Expectations:}

1. c. Students will demonstrate skill in researching questions, problems and issues.

- Students will examine background information.

- Students will identify consequences.

Materials and Resources Required:

See Lesson 13.

\section{Student Preparation:}

Have the students review the bugs and their names. Have them identify each of the five bugs in a sample. Explain that there will likely be many other kinds of bugs in the samples but that you are only looking for the five invertebrates identified. 


\section{Instruction Strategy:}

1. Review the relative oxygen requirements of the five invertebrates.

2. Explain that you have two samples of invertebrates from the river bottom. Explain that one sample was taken from below the local water treatment plant, and that the other was taken from above the plant.

3. Explain the effect of sewage on the oxygen levels in the water. Background Information Pages 2-6, AIM by FEESA.

4. The riddle the class must solve is where did the samples $A$ and $B$ come from.

5. Divide the class into pairs, with each pair counting bugs in their portion of a sample. Have students counting sample A combine their results and have students counting sample $B$ combine their results.

6. Examine results.

\section{Student Summary and Follow-Through:}

Have students discuss the effects of this reduced oxygen level on organisms other than invertebrates (i.e. effects on fishing, birds, etc.). What additional factors in the effluent from the sewage treatment plant could be affecting the invertebrates?

\section{Evaluation:}

Formative - Participation

\section{Unit Name: River Tripping}

Element Name: Environmental Investigations

Lesson Subject: Aquatic Invertebrate Monitoring - Verifying the Results

Lesson: 16

\section{Specific Learner Expectations:}

1. C. Students will demonstrate skill in researching questions, problems and issues.

\section{Materials and Resources Required:}

Plan a canoe trip that will include a section of a river below the local water treatment plant. Take samples from a number of sites below the plant to see if the oxygen levels return to the levels found above the plant. The more samples you have time to collect the more conclusive your results will be.

\section{Student Preparation:}

1. Explain the pantyhose, dual sampling kick method of aquatic invertebrate monitoring.

2. Have students examine one sample to see if they can do a quick qualitative analysis of where the oxygen levels drop off and rise again. Option: For a greater challenge have students do a quantitative analysis.

\section{Instruction Strategy:}

1. Have students follow the same sampling and counting procedures as in lessons 13 and 14 for each site sampled.

2. Plot the results on a graph. The greater the number of sites sampled the more accurate the graph will be. 
Student Summary and Follow-Through:

Have students write a brief description of where they would go fishing below the treatment plant and why. Would they eat the fish? Why not?

\section{Evaluation:}

Summative - Have students identify in their journals how they can reduce their water consumption.

\section{Unit Name: River Tripping}

Element Name: Outdoor Expeditions

\section{Lesson Subject: Canoe Trip Planning}

Lesson: 17

\section{Specific Learner Expectations:}

3. Students will demonstrate the knowledge and skills required for safe, comfortable outdoor living.

a. Students will select personal and group gear for outdoor activities:

- Students will select personal equipment for outdoor expeditions with particular consideration to maintaining body temperature and protection from injury.

- Students will select group equipment for outdoor expeditions to meet requirements for food, shelter, travel, emergencies.

- Students will demonstrate the proper care and maintenance of outdoor equipment.

b. Students will make preparations to meet food needs during extended outdoor activities by demonstrating an understanding of:

- Nutrition requirements.

- Portability and preservation.

- Food preparation techniques (e.g., cooking, fires and stoves).

\section{Materials and Resources Required:}

Personal and group equipment for trip

Completed parental permission forms

Equipment checklists

\section{Student Preparation:}

Discuss importance of proper planning.

\section{Instruction Strategy:}

Have the students finalize their menu and equipment needs.

Have students check their tents and stoves.

\section{Summary and Follow-Through:}

Review equipment checklists. 


\section{Evaluation:}

Summative - Students will be evaluated on how well prepared they are for the trip. Those students who cannot afford proper gear will be expected to find substitutes or let the teacher know of the difficulties they are having.

\section{Unit Name: River Tripping}

\section{Element Name: Outdoor Expeditions}

Lesson Subject: Canoe Tripping

Lesson: 18

\section{Specific Learner Expectations:}

Students will demonstrate skill, judgment, confidence and sensitivity through participation in a wide range of environmentally responsible activities in outdoor settings.

\section{Materials and Resources Required:}

Equipment for canoe trip

Volunteer leaders and drivers

Transportation to and from the river

\section{Student Preparation:}

Previous lesson

\section{Instruction Strategy:}

Never allow a situation to arise that is beyond your ability to ensure the safety of the students.

\section{Summary and Follow-Through:}

Set aside time each day to debrief the day and take time to debrief the entire trip. Working in smaller groups is more effective.

\section{Evaluation:}

Formative - Have students write in their journals what the trip meant to them (e.g. what were the high points, low points, did they see their particular animal or signs of it, did their tent and cooking group get along, what they would do differently next time). 
Unit Name: River Tripping

Element Name: Commitment To Action

Lesson Subject: Improving Habitat in the Community

Lesson: $19 / 20(a)$

Specific Learner Expectations:

3. Students will develop and act on plans that demonstrate responsibility for local and global environments.

Materials and Resources Required:

Resource Project Wild - p. 131; Local Fish and Wildlife officer.

\section{Student Preparation:}

Have students review wildlife survival requirements.

Instructional Strategy:

Follow procedures outlined on pp. 131-132 of Project Wild (Secondary Edition).

Student Summary and Follow-Through:

Have students report on the actions they took to improve habitat in their schoolyard or community.

Evaluation:

Summative - Evaluate student habitat improvement projects.

\section{ENVIRONMENTAL OPTION B}

Unit Name: River Tripping

Element Name: Commitment To Action

Lesson Subject: Monitoring and Reducing Water Consumption

Lesson: $19 / 20$ (b)

Specific Learner Expectations:

3. Students will develop and act on plans that demonstrate responsibility for local and global environments.

Materials and Resources:

Information on water conservation in the home. 


\section{Student Preparation:}

Have students examine ways they can conserve water at home and at school.

Instructional Strategy:

Have students select one action that they can take to conserve water and what the most effective means would be to meet that goal.

\section{Student Summary and Follow-Through:}

How can students pass this information on to other people in their community?

\section{Evaluation:}

Summative - Student activity reports on their efforts to reduce water consumption will be evaluated. 


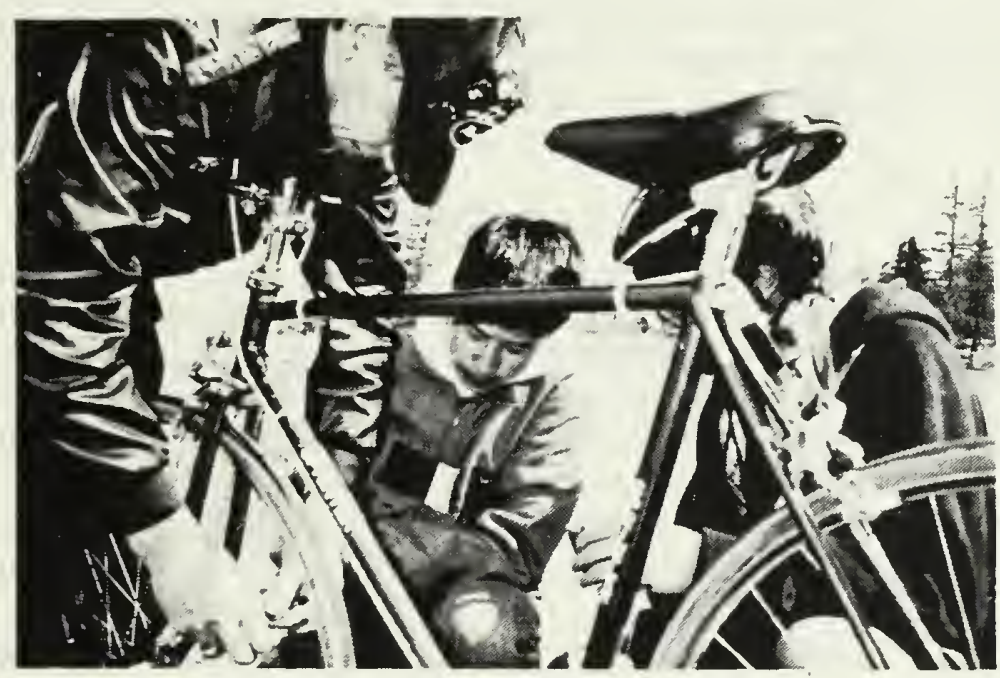

Chapter

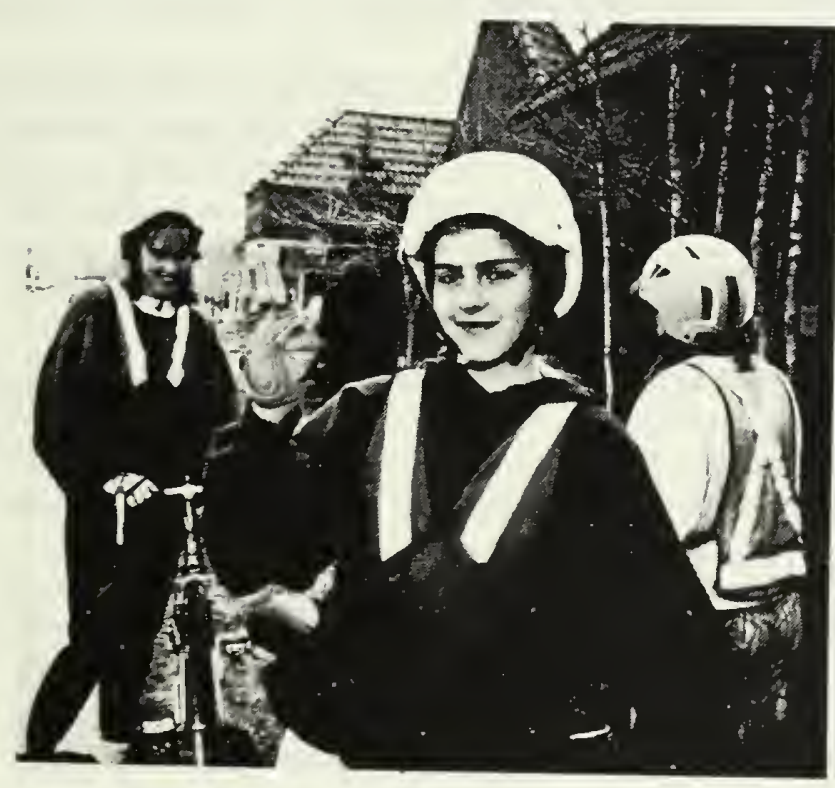


•

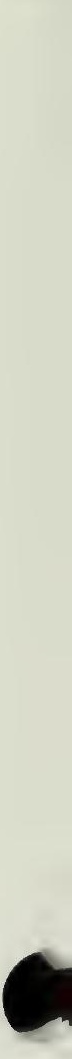




\section{PLANNING AND CONDUCTING SAFE OUTDOOR TRIPS}

Planning for safe outdoor trips can be viewed as a four-stage process: risk anticipation and preparation, ongoing risk assessment and risk reduction, emergency response and post trip follow-up. Educational expeditions in the outdoors are recommended and encouraged as an integral part of the Environmental and Outdoor Education course. However, teachers and students should be engaging in activities commensurate with their level of training and ability. Safety is not merely following correctly procedures and policies; it involves exercising situation-specific judgment throughout the program. As judgment is the product of experience, students should have the opportunity to make mistakes in a guided-discovery teaching situation. However, the teacher or leader should never permit a situation to arise that is beyond his or her ability to ensure the well-being of the student.

\section{RISK ANTICIPATION AND PREPARATION}

The preparation stage is the most important stage of any trip. Many of the mistakes commonly made while on the trail can be avoided, or anticipated and moderated at this stage. For example, if a group is familiar with hypothermia they are more likely to be dressed properly, reducing the risk of getting cold and wet. If they are familiar with the signs of hypothermia before an incident, they are more likely to recognize that their core temperature is dripping before it becomes a critical problem. And finally, if hypothermia does become a critical problem they will have the knowledge, skills and equipment to treat it properly. The preparation stage includes:

1. Pre-trip communication and administration.

2. Pre-trip logistics.

3. Student preparation.

1. Pre-trip Communication and Administration Wherever possible samples of forms, introductory letters, contact lists, sources of information on courses, equipment and expertise, and reference materials will be included.

a. Trip administration includes:

- parental permission forms.

- health information forms.

- school/system authorization forms.

- accident report forms.

- route cards and evacuation routes.

b. Activities should conform to applicable laws and regulations regarding:

- trespassing.

- national park permits.

- restrictions on camping such as open fires, fishing regulations, size of group, etc.

c. Parents, school administration, park authorities or others who would be informed in the event of an accident or other circumstance should have:

- itineraries (route cards).

- participant lists.

- communication channels (e.g. contact lists for current rescue services in area).

- emergency response plans.

d. Board and school policies and practices should be understood by principals, teachers, parents, volunteers and students. These policies and practices should include:

- volunteer policies and expectations.

- insurance criteria for coverage.

- budget requirements (how to charge for a trip or year program). 
- supervision requirements.

- transportation requirements.

- educational criteria.

- authorization requirements.

- procedures in the event of a calamity.

- procedures for informing the media and others.

e. Students and parents should be informed about and understand potential hazards and risks either at pre-trip meetings or in a handout that goes home to parents.

f. Leaders should be familiar with the area in which the trip is being conducted.

2. Pre-trip Logistics

Some pre-trip logistics should be included in pre-trip communications that go out to parents and administrators (e.g. transportation arrangements).

a. Transportation considerations include:

- luggage space.

- meeting place and time.

- mode of travel.

- emergency vehicle.

- driver co-ordination/licensing.

- keys.

b. Equipment considerations include:

- equipment testing and setup (e.g. stoves, tents).

- group equipment needs.

- student equipment needs.

- rentals vs. purchases.

c. Facility considerations include:

- reservations.

- deposits.

- cancellation insurance.

d. Departure date considerations include:

- area policies (e.g. some parks check parking lots before shifts end).

- weather report and forecast.

- back country registration and permits.

- current area information (e.g. bear scares, potential slide areas).

e. Pre-trip training in a particular skill area (e.g. canoeing, skiing, backpacking, etc.).

3. Student Preparation

Teachers should ensure that students are prepared in the following areas:

a. Mental and physical fitness considerations include such questions as: are participants capable of undertaking the activity as initially planned, has the pre-trip training been extensive enough, is the trip reasonable (e.g. weight of packs, distance, elevation gain).

b. Medical considerations include past and present medical problems (e.g. diabetes, hypersensitivity, heart problems, breathing problems, etc.) and how student medication is to be administrated. 
c. Experience considerations include:

- student background/experience.

- background knowledge.

- Experience of other leaders and volunteers.

d. Equipment considerations should include checking packs before the trip to determine if the students have the proper gear (e.g. proper clothing and footwear).

e. Students should be given training in what procedures they should follow if they are lost or separated from the group.

f. Students will be expected to demonstrate the basic knowledge, skills and attitudes necessary for safe, comfortable, outdoor experiences of all kinds, in all seasons. It is important that the preparation and training is commensurate with the degree of difficulty of the trip. The competence required for safe, comfortable experiences in the outdoors are listed in the specific learner expectations in the outdoor strand.

\section{ONGOING RISK ASSESSMENT AND RISK REDUCTION}

Risk reduction is a stage that is dependent on judgment and experience. Judgment is the product of experience. Experience includes recognizing factors such as deteriorating weather, a tiring group or increasingly more difficult rapids, and including those parameters in the judgment process. It is important that the students are aware of the decisions a teacher makes so that they will gain experience in this critical area and eventually participate in the decision-making process. Many of the hazard recognition skills can be taught in the classroom in the preparation stage. For example, a student may never have seen a grizzly bear in the wild but after having seen a picture or video in the classroom he or she would be well aware of the risk involved and the means of reducing it.

A significant aspect of ongoing risk management and risk reduction is group management. The skills are:

1. Pacing (e.g. speed of travel, rest stops, distance travelled, awareness of fitness level of all participants).

2. Group control (e.g. lead and sweep, position of leader, regrouping procedures, signal systems, buddy systems).

3. Objective hazard recognition in the field (e.g. weather, terrain, flora and fauna).

4. Task allocation (e.g. shelter, cooking, water, waste disposal).

5. Group and organizational rules and norms.

6. Subjective hazard recognition in the field (e.g. level of group energy, level of co-operation).

Group size is an important consideration (e.g. potential environmental impact, level of supervision required, effective communication). 


\section{EMERGENCY RESPONSE}

Emergency response is the final stage of the Critical Situation Model. If the group has been well prepared and appropriate judgment has been exercised to this point, the teacher will have maximized the number of options they have to choose from to respond to an emergency situation. Proper recognition leads to a relatively short list of considerations in an emergency response situation:

1. Emergency Response Action Plan
a. Accident site approach considerations
b. Administering first aid
c. Signaling
d. Evacuation plan
e. External assistance procedure

2. Group management in an emergency situation
a. Student response to accident
b. Student involvement

\section{Post Trip Follow-up}

1. Integrating the experience
a. Trip journals
b. Debriefing
- Formal
- Informal

c. Recommendations for future trips

2. Celebrating the trip (newsletters, bulletin boards, slide shows)

3. Post-trip administration
a. Trip log update
b. Final evaluation 


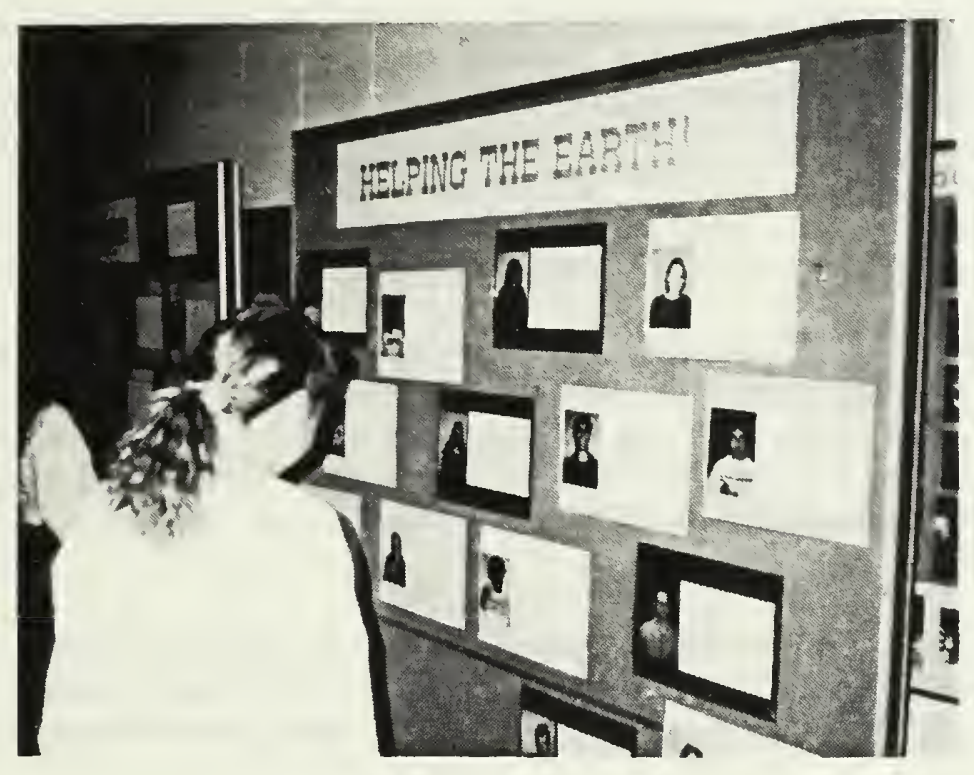

Chapter

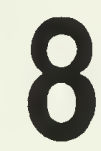

Evaluation •
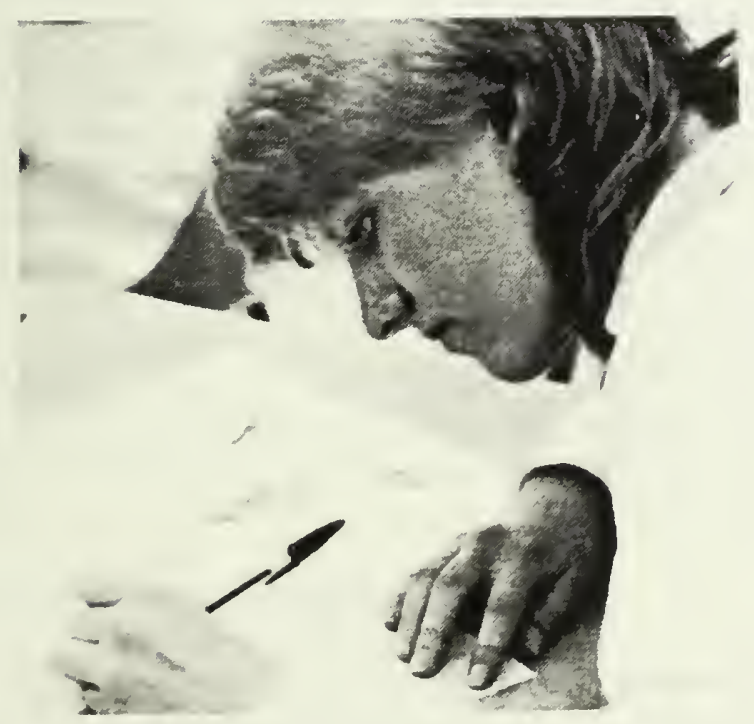


\section{EVALUATION TECHNIQUES}

Student evaluation should take the following into consideration:

a. general and specific learner expectations.

b. increasing independence and choice by students.

c. students being aware of course objectives and evaluation methods to be used.

d. developing a positive attitude toward a commitment to action in personal growth, environmental action and outdoor pursuits.

e. evaluation for success and for motivation to improve. Appropriate course requirements should be established and opportunities should be given for students, singly or in groups, to achieve a standard or course requirement. This may require that students repeat tests or assignments to meet course requirements.

f. that the evaluation component include an assessment of the student's processing abilities in addition to assessing the student's knowledge, skills and attitudes.

g. evaluation weighting should reflect the emphasis in instruction time.

There are two types of evaluation that may be used in this program: formative and and summative. Formative evaluation is the ongoing determination of the student attitudes, skills or level of understanding. These evaluations serve as an indicator to the teacher and the student where additional effort may be needed in the program. Summative evaluation is the determination of a mark based on a student's achievement of learner expectations. Any activity that is graded may become a component of the summative evaluation.

The Environmental and Outdoor Education course is designed to be holistic in nature with an aim of empowering the student. The course allows for a variety of learning styles to be accommodated. Therefore a wide variety of student evaluation methods must be used. These might include:

- Activity Reports

- Interviews

- Self-Evaluation

- Observation

- Case Studies

- Expedition Evaluation

\section{ACTIVITY REPORTS AND PROJECTS}

Activity reports or projects are assignments given to individual students or to a small group of students. Usually they involve research on a specific topic in the program. Activity reports or projects allow students some freedom to express individuality and to demonstrate particular strengths. A variety of resources can be incorporated into a project, for example:

- written report

- models

- audio-visua! naterial

- photographs

- drama

- drawings

- graphs

\section{Sample Report Format and Marking Guide}

Evaluation of reports will be based on guidelines provided by the teacher for preparing and presentation of the report. Following is a brief sample report format and a corresponding marking guide. 
Pick an environmental issue you are concerned about and the action which you plan to take.

How to go about it

1. Use the environmental investigation method to address the issue.

2. Consider at least three alternatives to the current practice.

3. Explain your choice.

4. What is your plan of action?

The Task

You will be given class time in the library to research the report. If you are not planning to submit a written report please talk to me.

Due Date

Marking

$40 \%$ Application of the environmental investigation process

$20 \%$ Consideration of alternatives - well researched

$10 \%$ Reasoning behind choice

$10 \%$ Action Plan

$10 \% \quad$ Organization and presentation

\section{INTERVIEW}

Understanding developed in this course may be difficult for some students to express in writing. Also, many of the problem-solving activities require that teachers observe and evaluate the process rather than the outcome. For these reasons, an interview format is one of the evaluation formats to be considered for use with this course. This approach can be used to particular advantage by the EOE teacher, as he or she often has a greater degree of rapport with students as a result of the many commonly shared experiences in the outdoors.

The teacher should approach the interview with a series of questions that can be used as a guideline. The teacher should use additional probing questions when appropriate. An interview may be focused on a problem-solving assignment such as an environmental investigation. The teacher may pose the following questions regarding an environmental investigation:

1. Identification and understanding of the problem:

- Can you describe the problem in this investigation?

2. Gathering additional information:

- How did you go about getting more information on this problem?

3. Alternatives:

- What would you do at this point in the investigation?

4. Choosing:

- What criteria would you use to make a decision?

5. Action:

- How would you evaluate the outcome of your action?

It is important to be patient and wait for answers. If the student can't remember something, ask the student to respond to a hypothetical situation. 


\section{SELF-EVALUATION}

Self-evaluation is an important evaluation technique, especially as this program is designed to empower students to act and learn from actions beyond the school. For lifelong learning the habit of selfevaluation is required. A sample self-evaluation questionnaire follows:

Please rate your performance on a scale of 1 to 5 .

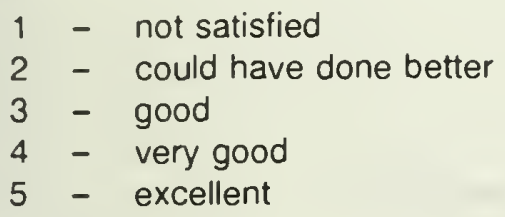

Knowledge and Skills

1. Care of equipment

2. Fire-building skills

3. Recognition of hazards

4. Shelter-building skills

5. Ability to listen without interrupting

6. Respect for other people's ideas

7. Respect for the environment

8. Appropriate use of clothing (i.e. thermoregulation)

9. Map and terrain interpretation skills

10. Willingness to help others

$\begin{array}{lllll}5 & 4 & 3 & 2 & 1 \\ 5 & 4 & 3 & 2 & 1 \\ 5 & 4 & 3 & 2 & 1 \\ 5 & 4 & 3 & 2 & 1 \\ 5 & 4 & 3 & 2 & 1 \\ 5 & 4 & 3 & 2 & 1 \\ 5 & 4 & 3 & 2 & 1 \\ 5 & 4 & 3 & 2 & 1 \\ 5 & 4 & 3 & 2 & 1 \\ 5 & 4 & 3 & 2 & 1\end{array}$

In follow-up to self-evaluations, teachers may want to have students compare their evaluations to those of the teacher. If the teacher and the student each fill out the evaluation form, follow-up discussion may focus on areas of greatest discrepancy between teacher and student evaluations. The self-evaluation should be followed with recommendations for future action.

\section{OBSERVATION}

Observation is an important evaluation tool in an experientially oriented program such as Environmental and Outdoor Education. When evaluating, the following guidelines and suggestions may be helpful:

1. Develop a strategy that allows you to focus on a small group of students (e.g. cooking a meal, setting up a tent, performing an initiative task).

2. Have specific criteria in mind (e.g. listening to others without interrupting, willingness to help, shows respect for ideas of others).

3. Make notes or keep informal records of what has happened (e.g. yes/no checklist, a tally, frequency of action, numerical rating scale, anecdotal record, p. 62).

4. Develop a checklist to help you concentrate on the things you want to observe.

5. In follow-up discussions with students regarding their evaluation, emphasize that you are evaluating student performance and not the student. Having notations of concrete examples of student performance can be especially helpful.

\section{CASE STUDIES}

Case studies can be used to bring a variety of situations to the classroom setting. Teachers should be prepared for more than one answer and expect students to justify those answers orally or in writing. It is important that the case study has enough information so that the students have a clear picture of the 
problem. It is also important that students state the assumptions they are making when they respond to the study.

\section{EXPEDITION EVALUATION}

Student evaluation on expeditions can take a number of forms. Criteria to be evaluated include:

- proper preparation

- participation

- individual contribution to trip

- co-operation with peers/group leader

- following safety guidelines

- performance of specific tasks (e.g. cooking, tents, hygiene, navigation)

Other evaluation techniques that may be used include:

Certification (e.g. first aid)

Awards

Extra-curricular project and events (e.g. Environment Week celebrations)

Simulations (e.g. computer, first aid)

Peer evaluation

Parent evaluation

Written tests

Checklists and rating scales

Research and investigations

Role playing and analogies

Displays, bulletins, bulletin boards

Subjective evaluation

Skill development and improvement (e.g. cross-country skiing, fitness)

Questionnaires

Small group evaluation

Autobiographies

Alternate projects (e.g. unable to attend expedition - alternate assigned)

General safety practices and attitudes

Anecdotal records by teacher or student

Readings to small groups or class

News bulletins 
Appendices 
$\bullet$

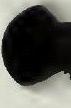




\section{BACKGROUND}

Controversial issues are those topics which are publicly sensitive and on which there is no consensus of values or belief. By their nature, controversial issues generate diverse opinions and debate on the distinctions between right and wrong, justice and injustice, and on interpretations of fairness and tolerance. They include topics on which reasonable people may sincerely disagree.

Opportunities to deal with sensitive issues and topics are an integral part of the education programs and schooling process in Alberta. Alberta Education recognizes that education cannot remain neutral on all issues or avoid all topics that are controversial. Alberta Education also recognizes that courses of study and education programs offered in Alberta schools must handle controversial issues in a manner that respects the rights and opinions reflected in different perspectives, but that rejects extreme or unethical positions.

For sound judgments to be made, students should have experiences in selecting, organizing and evaluating information. The educational benefits to be gained by studying controversial issues include the development of critical thinking, moral reasoning, and an awareness and understanding of contemporary society.

\section{POLICY}

Alberta Education believes that studying controversial issues is important in preparing students to participate responsibly in a democratic and pluralistic society. Such study provides opportunities to develop students' capacities to think clearly, to reason logically, to open-mindedly and respectfully examine different points of view, and to reach sound judgments.

\section{LEGISLATION}

\section{School Act}

25(1) The Minister may by order do the following:

(c) subject to the rights of a board to provide religious instruction, prohibit the use of a course, education program or instructional material in schools; ... .

Other legislation:

Alberta Bill of Rights, R.S.A. 1980, Chapter A-16

Canadian Charter of Rights and Freedoms, Constitution Act, 1982

The Ministerial Order under section 25(1)(d) of the Act as cited in the Ministerial Orders and Directives section of this Policy Manual.

\section{PROCEDURES}

1. Sensitivity on the part of teachers, students and other participants in controversial issues shall be exercised to ensure that students and others are not ridiculed, embarrassed, intimidated or degraded for positions which they hold on controversial issues.

2. Information regarding controversial issues should: 

a. represent alternative points of view, subject to the condition that resources used are not restricted by any federal or provincial law;
b. appropriately reflect the maturity, capabilities and educational needs of the students;
c. meet the requirements of provincially prescribed and approved courses of study and education programs; and
d. reflect the neighbourhood and community in which the school is located, as well as provincial, national and international contexts.

3. Controversial issues which have been pre-planned by the teacher and those which may arise incidentally in the course of instruction should be used by the teacher to promote critical inquiry rather than advocacy, and to teach students how to think rather than what to think.

4. The school should play a supportive role to parents in the areas of values and moral development, and shall handle parental decisions in regard to Controversial Issues with respect and sensitivity.

Alberta Education Policy Manual. 1989. 


\section{APPENDIX B: SUPPLEMENTARY INFORMATION SOURCES}

\section{INFORMATION SERVICES}

ECA Online (Computer Network) - a computerized conference, message service and environmental information bulletin board. Parameters: $300-1200$ baud, 8 data bits, 1 stop bit, no parity at 438-5793.

ENERGY MATTERS Inquiry Line - energy experts and a computer data bank provide information on how energy can be used wisely in the home. If you have any questions on anything related to home energy, DIAL "0," and ask for Zenith 22339 (Edmonton, 427-5300). Publications are available on topics ranging from heating systems to caulking and weatherstripping. You can also use ENERGY MATTERS Line to find out about other residential services offered by the Energy Efficiency Branch.

Energy Efficiency Branch

Alberta Energy

2nd Floor, 10010-106 Street

Edmonton, Alberta

T5J 3L8

427-5200 


\section{FIELD TRIP CENTRES}

The following is a partial list of centres that provide hands-on experience for students in an aspect of energy conservation or resource conservation. Contact should be made directly with the resource centre to obtain details of what is offered to school groups or students.

Alberta Forest Service Museum

Forest Technology School

1176 Switzer Drive

Hinton, Alberta TOE 1 BO

865-8211

Alberta Tree Nursery and Horticulture Centre

R.R. \#6, 17507 Fort Road

Edmonton, Alberta T5B $4 \mathrm{~K} 3$

Telephone: $\quad 422-1789$ (in Edmonton) 198-5272 (toll-free through any government R.I.T.E. Operator check your local telephone directory under "Government of Alberta").

Bennett Environmental Education Centre Edmonton Public Schools 9703-94 Street

Edmonton, Alberta T6C $3 W_{1}$

468-1439

\section{Calgary Zoo}

Botanical Gardens and Prehistoric Park

P.O. Box 3036, Station "B"

Calgary, Alberta T2M 4R8

265-9310

OR Zoo located at:

1300 Zoo Road N.E., Calgary, AB

Devonian Botanic Garden

University of Alberta

Edmonton, Alberta T6G 2E1

987-3054

OR located at:

Hwy $60,15 \mathrm{~km}$ south of Hwy 16

Environmental Resource Centre

10511 Saskatchewan Drive

Edmonton, Alberta T6E 4S1

433-4808

\author{
Energeum \\ (Energy Resources Conservation Board) \\ 640-5th Avenue S.W. \\ Calgary, Alberta T2P 3G4 \\ $297-4293$
}

Fort Calgary Preservation Society

P.O. Box 2100, Station "M", \#106

Calgary, Alberta T2P $2 \mathrm{M} 5$

290-1875

OR office located at:

750-9th Avenue S.E., Calgary, AB

Ft. McMurray Oil Sands Interpretive Centre 515 MacKenzie Blvd.

Ft. McMurray, Alberta T9H $4 \times 3$

743-7167

Glenbow Museum

130-9th Avenue S.E.

Calgary, Alberta T2G 0P3

264-8300

John Janzen Nature Centre

P.O. Box 2359

Edmonton, Alberta T5J 2R7

434-7446

OR located at:

Fort Edmonton Park, Fox Drive 


\section{TECHNICAL REPORTS}

The following list of technical reports may provide you with more detail on energy and conservation projects.

Alberta Agriculture. 1987. Low Energy Home Designs. Edmonton: Alberta Agriculture.

American Section of the International Solar Energy Society. 1976. Sharing the Sun! Solar Technology in the Seventies, ed. K.W. Boer. Cape Canaveral, Florida.

Calhoun, Elizabeth. 1983. 20 Simple Solar Projects. Emmaus, Pennsylvania: Rodale Press.

Energy Resources Conservation Board. 1987. Energy Alberta 1987. Calgary.

McCallum, Bruce. 1979. Environmentally Appropriate Technology: Renewable Energy and Other Developing Technologies for a Conserver Society in Canada 4th ed. Ottawa: Office of the Science Advisor, Fisheries and Environment Canada.

Nova Scotia Department of Mines and Energy. 1981. One Hundred and One Solar Energy Projects. Halifax. 


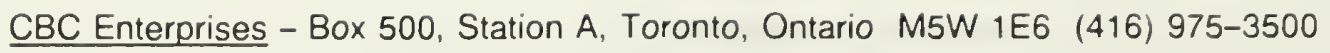

Wonderstruck: Environment Alert

The Journal: The Greenhouse Effect

The Journal: Looting A Legacy

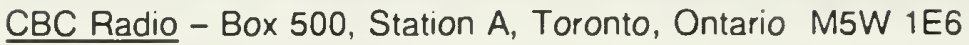

David Suzuki's Matter of Survival series of audiotapes $\$ 10$ each or series of five for $\$ 45$.

Cheques payable to CBC Radio.

Environment Council Library, Environment Council Library, Weber Centre, 5555 Calgary Trail,

Edmonton, Alberta, telephone 427-5793.

One Man's Garbage, Another Man's Gold - video, 15 minutes.

Petroleum Resources Communication Foundation, 1250, 633-6th Avenue S.W., Calgary, AB T2P 2 Y5.

Alaska Oil Spill - The Veco Response - describes the clean-up of the Exxon Valdez oil spill.

One Onion at a Time - shows the importance of finishing high school, and of taking math and science to lead to many careers in the petroleum industry.

Trans-Alta Utilities Corp. - 110-12th Avenue S.W., Box 1900, Calgary, AB T2P 2M1 267-7459 (Cheryl Corbill).

\section{Acid Rain}

Careers in the Electrical Industry

University of Alberta - Educational Media Services, Corbett Hall, 82 Avenue and 112 Street, Edmonton, AB T6G 2G4, 492-5039 (5040).

A Planet For The Taking - No. 1: "Human Nature"

The Journal: The Greenhouse Effect 


\section{SPECIAL INTEREST GROUPS - for Students}

The following is a list of groups, organizations and associations that students can become involved with as part of their personal commitment to conservation and environmental protection. Services having a fee associated with them are designated with a (\$) sign.

Organization

Alberta Forestry Association (\$)

101 Alberta Block, 10526 Jasper Avenue

Edmonton, Alberta T5J 127

428-7582

Calgary Zoo (\$)

Botanical Gardens and Prehistoric Park

P.O. Box 3036, Station "B"

Calgary, Alberta T2M 4R8

265-9310

OR Zoo located at:

1300 Zoo Road N.E., Calgary, AB

Canadian Coalition on Acid Rain

112 Street and St. Clair Avenue W

Suite 401

Toronto, Ontario M4V 2 Y3

Camrose International Institute (\$)

4802-49 Avenue

Camrose, Alberta T4V OM7

672-8780

Canadian Water Resources Association (\$)

Alberta Branch

48 Baker Crescent, N.W.

Calgary, Alberta T2L 1R4

282-0405

Earth Island Institute (\$)

300 Broadway, Ste. 28

San Francisco, CA 94113

U.S.A.

(415) $788-3666$

Edmonton Recycling Society

11631-80 Street

Edmonton, Alberta T5B 2N3

471-0071

\section{Resources}

- Volunteers accepted

- Members accepted in zoological society (\$)

- Quarterly magazine with membership

- Special activities

- Information packages

- Encourage formation of support groups

- Student memberships (\$)

- Free newsletter

- Memberships (\$)

- Newsletter with membership

- Student memberships (\$)

- News magazine for members

- Volunteers accepted for projects

- Volunteers accepted 
Environment Week Association

642-21, 10405 Jasper Avenue

Edmonton, $A B$ T5J $3 S 2$

427-6310

OR office located at:

9820-106 Street, 12th Floor (interim)

Edmonton, Alberta T5K 2J6

Environmental and Outdoor Education Council The Alberta Teachers' Association

Barnett House

11010-142 Street

Edmonton, $A B$ T5N 2R1

453-2411

Environmental Resource Centre

10511 Saskatchewan Drive

Edmonton, AB T6E 4S1

$433-4808$

Ft. McMurray Oil Sands Interpretive Centre 515 MacKenzie Blvd.

Ft. McMurray, AB T9H $4 \times 3$

743-7166

Friends of the Earth $(\$)$

Suite 701, 251 Laurier Avenue W

Ottawa, ON K1P 5J6

(613) 230-3352

Friends of Environmental Education

Society of Alberta (FEESA)

641-21, 10405 Jasper Avenue

Edmonton, $A B$ T5J $3 \mathrm{~S} 2$

427-6210

Green Teacher

95 Robert Street

Toronto, ON M5S $2 \mathrm{~K} 5$

(416) 960-1244

Greenpeace Foundation (\$)

1711B-16 Street N.W.

Calgary, AB T2M 3P1

$261-4828$

$\mathrm{OR}$

10511 Saskatchewan Drive

Edmonton, AB T6E 2S1

433-4808
- Teacher/school memberships (\$)

- One free newsletter per school

- Referral to many different youth-oriented activities from many organizations

- Memberships (\$)

- Newsletter and journal

- Annual conference

- Travelling library

- Travelling library

- Resources available on environmental issues

- Information on site

- Speakers available

- Hands-on activities

- Information packages

- Videos for loan

- Field trips

- Teaching resources and audiovisuals

- Regular and student memberships

- Newsletter with membership

- Teacher memberships (\$)

- Newsletter

- Teaching kits (\$)

- Energy issues/ecology materials for classroom

- Magazine (\$)

- Resource material

- Regular and student memberships (\$)

- Magazine with membership

- Speakers' bureau

- Videos 


\section{CANADIAN ENVIRONMENTAL ORGANIZATIONS AND GOVERNMENT AGENCIES}

Alberta Environment Network 10511 Saskatchewan Drive Edmonton, $\mathrm{AB}$ T6E 4S1

(403) 433-9302

Alberta Wilderness Association Box 6398, Station D

Calgary, AB T2P 2E1

(403) 283-2025

Atlantic Environment Network 180 St. John Street Fredericton, NB E3B 4 A9 (506) 453-0680

B.C. Environmental Network 2150 Maple Street Vancouver, BC V6J 3T3

(604) $733-2400$

Canadian Arctic Resources Committee 111 Sparks Street

Ottawa, ON K1P 585

(613) 236-7379

Canadian Coalition on Acid Rain 112 St. Clair Avenue, West, \#401 Toronto, ON M4V 2 Y3 (416) 968-2135

Canadian Environment Network P.O. Box 1289, Station B Ottawa, ON K1P 5R3

(613) 563-2078-3337

Canadian Environmental Law Association 243 Queen Street West, 4th Floor Toronto, ON M9N $2 \mathrm{H} 8$ (416) 960-2284

Canadian Nature Federation 453 Sussex Drive Ottawa, ON K1N $6 Z 4$

(613) 238-6154

Canadian Organic Growers Box 6408. Station J Ottawa, ON K2A 3 Y6
Catalyst Education Society

P.O. Box 99

Lillooet, BC VOK 1 VO

Connexions

427 Bloor Street West

Toronto, ON M5S $1 \times 7$

(416) 960-3903

Conservation and Renewable Energy Industry Council

Suite 209, 135 York Street

Ottawa, ON K1N 5T4

Ducks Unlimited Canada

1190 Waverly Street

Winnipeg, MB R3T 2E2

(204) 477-1760

Ecology Action Centre of Nova Scotia 3115 Veith Street, 3rd Floor

Halifax, NS B3K 3 G9

(902) 454-7828

Energy Probe

225 Brunswick Street

Toronto, ON M5S $2 \mathrm{M} 6$

Federation of Ontario Naturalists

355 Lesmill Road

Don Mills, ON M3B 2W8

(416) 444-8419

Friends of the Earth

251 Laurier Avenue West, \#701

Ottawa, ON K1P 5J6

(613) 230-3352

Greenpeace

578 Bloor Street West

Toronto, ON M5G $1 \mathrm{~K} 1$

(416) 538-6470

Harmony Foundation

19 Oakdale Avenue

Ottawa, ON K1Y 353 
Islands Protection Society

Box 688

Queen Charlotte City, BC VOT 1SO

Manitoba Environment Network

P.O. Box 3125

Winnipeg, MB R3C 4E6

(204) 956-1468

Manitoba Naturalists Society

\#302-128 James Avenue

Winnipeg, MB R3B ON8

(204) 943-9029

Northwest Wildlife Preservation

P.O. Box 34129, Station D

Vancouver, BC V6J $4 \mathrm{~N} 3$

(604) 736-8750

Outdoor Recreation Centre of B.C.

336-1367 W. Broadway

Vancouver, BC V6H 4 A9

(604) 737-3058

Ontario Environment Network

P.O. Box 125, Station P

Toronto, ON M5S 2 S7

(416) 925-1322

Pollution Probe Foundation

12 Madison Avenue

Toronto, ON M5R 1S1

(416) 926-1907

Rainforest Action Society

P.O. Box 46695, Station G

Vancouver, BC V6R 4K8

(604) 734-7248

Recycling Council of Ontario

P.O. Box 310, Station P

Toronto, ON M5S 2S8

(416) 960-1025

Roseau Quebecois des Groupes Ecologistes CP 1480, Place d'Armes

Montreal, PQ H2Y $3 \mathrm{~K} 8$

(514) 982-9444
Saskatchewan Eco-Network 205-219 22nd Street East Saskatoon, SK S7K OG4 (306) 665-1915

Sea Shepherd Conservation Society P.O. Box 48446, Bentall Centre Vancouver, BC V7X $1 \mathrm{~A} 2$

(604) 688-7325

Sierra Club of Ontario 2316 Queen Street East Toronto, ON M4E $1 \mathrm{G} 8$ (416) 698-8446

Western Canada Wilderness Committee 20 Water Street

Vancouver, BC V6B 1 A4

(604) 683-8220

Wildlife Habitat Canada 1704 Carling Avenue, \#301

Ottawa, ON K2A 1 C7

(613) 722-2090

World Wildlife Fund Canada 60 St. Clair Avenue East Toronto, ON M5T 1 N5 (416) 923-8173

\section{Environment Canada,} Communications Directorate

National Inquiries Centre Terrasses de la Chaudiere Ottawa, ON K1A OH3

(819) 997-2800

Atlantic Region 15th Floor, 45 Alderney Drive Dartmouth, NS B2Y $2 \mathrm{~N} 6$

(902) 426-1930

Quebec Region 3 Baude Street P.O. Box 606 Quebec, PQ G1R 4V7 (418) 648-7204 
Ontario Region

25 St. Clair Avenue East

Toronto, ON M4T $1 \mathrm{M} 2$

Western and Northern Region

Twin Atria \#2, 2nd Floor

4999-98 Avenue

Edmonton, $A B$ T6B $2 \times 3$

(403) 468-8074

Pacific and Yukon Region

3rd Floor, Capilano 100

Park Royal South

W. Vancouver, BC V7T 1 A2

(604) 666-5900

\section{Environmental Partners Fund}

Alberta

Twin Atria \#2, 2nd Floor

4999-98 Avenue

Edmonton, $\mathrm{AB}$ T6B $2 \times 3$

(403) 468-5887

Manitoba

457 Main Street

Winnipeg, MB R3B 3E8

(204) 983-2110

Newfoundland

P.O. Box 5037

St. John's, NF A1C 5 V3

(709) $772-5488$

New Brunswick

527 Queen Street, Box 400

Fredericton, NB E3B $4 Z 9$

(506) 452-3286

Northwest Territories

P.O. Box 2970

Yellowknife, NT X1A 2R2

(403) 920-8500

Nova Scotia

15th Floor, 45 Alderney Drive

Dartmouth, NS B2Y 2 N6

(902) 426-6473
Ontario

25 St. Clair Avenue East

Toronto, ON M4T $1 \mathrm{M} 2$

(416) 973-6467

Prince Edward Island

P.O. Box 426

Charlottetown, PE C1A 7K

Quebec

P.O. Box 6060, Hauteville

Quebec, PQ G1V $4 \mathrm{H} 5$

(418) 648-4296

Saskatchewan

241-1901 Victoria Avenue

Regina, SK S4P 3R4

(306) 780-6002

Yukon

\#101-204 Range Road

Whitehorse, NT Y1A 3V1

(403) 667-3400

\section{Provincial Environment Departments}

\author{
Alberta \\ Department of Environment \\ 14th Floor, Oxbridge Place \\ 9820-106 Street \\ Edmonton, $A B$ T5K 2J6 \\ (403) 427-6236 \\ British Columbia \\ Ministry of Environment \\ Parliament Buildings \\ Victoria, BC V8V $1 \times 5$ \\ (604) 387-5669 \\ Manitoba \\ Department of Environment \\ Room 350 Legislative Building \\ 450 Broadway Avenue \\ Winnipeg, MB R3C OV8 \\ (204) 945-3522
}


New Brunswick

Department of Municipal Affairs and Environment 2nd Floor, 364 Argyle Street

P.O. Box 6000

Fredericton, NB E3B $5 \mathrm{H} 1$

(506) 453-2558

Newfoundland

Department of Environment

Confederation Building, West Block

4th Floor, P.O. Box 4750

St. John's, NF A1C 5T7

Nova Scotia

Department of Environment

Terminal Road Building

5151 Terminal Road, 5th Floor

P.O. Box 2107

Halifax, NS B3J 3B7

(902) 424-5300

Ontario

Ministry of Environment

135 St. Clair Avenue W., 14th Floor

Toronto, ON M4V 1 P5

(416) $323-4272$

Quebec

Ministere de l'Environment

3900, rue Marly, 6 etage

Ste-Foy, PQ G1X 4E4

(418) 643-7860

Saskatchewan

Department of Environment

Room 214, Walter Scott Building

3085 Alberta Street

Regina, SK S4S OB1

(306) 787-6111

Northwest Territories

Department of Renewable Resources

P.O. Box 1320

Yellowknife, NT X1A 2L9

Yukon Territories

Department of Renewable Resources

P.O. Box 2703

Whitehorse, YT Y1A 2 C6

(403) 667-5877 


\section{Organizations and Guides Offering Lesson Plans and Sc ool Activities}

Alliance of Environmental Educators has a variety of environmental education curricula. 2.111 Wilson Blvo., Suite 701, Arlington, VA 22201.

American Nature Study Society promotes environmental education through "Nature Study" magazine, meetings, workshops and field trips. 5881 Cold Brook Rd., Homer, NY 13077. Telephone: (607) 7493655.

Biological Science Curriculum Study (BSCS). A variety of materials for teachers and students including textbooks, laboratory manuals, subject modules and films. Contact BSCS, The Colorado College, Colorado Springs, CO 80903. Telephone: (303) 473-2233.

Conservation and Renewable Energy Information Referral Service. Public inquiries and bibliographies on renewable and non-renewable energy. Telephone: 1-(800) 523-2929.

Global Tomorrow Coalition has environmental education curricula on tropical forests, marine and coastal pollution and other global issues. 1325 B-Street, N.W., Washington, DC 20005. Telephone: (202) $628-4016$.

Institute for Earth Education. "Earthkeepers" is an educational program for helping young people live in harmony with the earth and other resources. P.O. Box 288, Warrenville, IL 60555.

National Geographic Society. Two filmstrip sets about energy and pollution: "Challenges to a Healthy Environment" (Advanced) and "This World of Energy: II" (Intermediate and Advanced). Educational Services, Dept. 90, Washington, DC 20036.

National Audubon Society publishes newsletters, newspapers and posters for a fee. New unit available on wetlands. Education Division, 950 Third Ave., NY, NY 10022. Telephone: (212) 832-3200.

National Wildlife Federation nature education catalogue lists science activity series, project kits, books, videos, etc. Attn: School Programs, 8925 Leesburg Pike, Vienna, VA 22184-0001.

Science and Environmental Education Resource Guide, 1989 directory of national science and environmental education resources. California State Department of Education, 721 Capital Mall, Sacramento, CA 95814.

Sierra Club provides free teachers' newsletter, list of environmental education materials, filmstrips, slides and videos, and a literature list for children. 703 Polk St., San Francisco, CA 94109.

Telephone: (415) 776-2211.

U.S. Environmental Protection Agency directory of environmental education materials for grades K-12. Office of Community and Intergovernmental Relations, Mail Code A-108 EA, U.S. Environmental Protection Agency, 401 M Street, SW, Washington, DC 20460. Telephone: (202) 382-4454.

Zero Population Growth teacher training programs and K-12 curriculum. Population Growth Education Program, 1400 16th Street, NW, Suite 320, Washington, DC 20036. Telephone: (202) 332-2200.

\section{Lesson Plan and School Activities}

A-Way with Waste curriculum guide about recycling for grades $K-12$. Washington Dept. of Ecology, 350 150th Ave., NE, Redmond, WA 98052. 
California State Environmental Education Guide (1987). Eight instructional units and six action projects $(\mathrm{K}-6)$. (\$17.95) Alameda County Office of Education, Library Dept., EG, 313 W. Winton Avenue, Hayward, CA 94544-1198.

California Energy Extension Service - Energy Action in Schools. Annotated bibliography of sample energy education materials, K-6 and 7-12. Governors Office of Plannıng and Research, 1400 10th Street, Room 209, Sacramento, CA 95814.

Green Box. Set of 178 activity cards for environmental educators $(K-8)$. Cost is approx. \$40. Contact Humboldt County Office of Education, Environmental Education Program, 901 Myrtle Avenue, Eureka, CA 95501. Telephone: (707) 445-5411.

Living Lightly in the City (K-6). Living Lightly on the Planet (7-12). Schlitz Audubon Centre. 1111 East Brown Deer Road, Milwaukee, WI 53217. Telephone: (414) 352-2880.

Outdoor Biology Instructional Strategies (OBIS). Set of activity cards for grades 4-9. Contact Delta Education, Inc., P.O. Box M, Nashua, NH 03061.

Project WILD wildife education program for school teachers. Western Regional Environmental Education Council, Boulder, CO; Western Association of Fish and Wildlife Agencies (WAFWA); or your state fish and game department.

Project Learning Tree (PLT) activity manual and teacher training workshops for environmental educators $(K-12)$ focusing on plant life. American Forest Institute, 1619 Massachusetts Ave., NW, Washington, DC 20036.

Rainbow Child Programs (K-6). Earth awareness lesson plans and teacher training workshops. Rainbow Child Programs, 1705 Balsam Lane, Villanova, PA 19085. Telephone: (215) 525-4133.

Sharing Nature with Children and Listening to Nature. Two books by Joseph Cornell Workshops. Ananda Publications, 14618 Tyler Foote Rd., Nevada City, CA 95959. Telephone: (916) 292-3225 or (800) 843-5272.

Toxics in My Home? You Bet! Curriculum units on Household Hazardous waste, $\mathrm{K}-12$ (also in Spanish) Golden Empire Health Planning Centre, 2100 21st Street, Sacramento, CA 95818. Telephone: (916) 731-5050.

Toxics: Taking Charge. A unit for grades 4-6. Supplement to the California State Environmental Education Guide. Alameda County Office of Education, 313 W. Winton Avenue, Hayward, CA 94554 1198.

Films, Videos and Plays

Bullfrog Films, Inc. rents films and videos with environmental themes. Bullfrog Films, Oley, PA 19547. Telephone: (215) 779-8226.

Corporation for Public Broadcasting 1990-Year of the Environment-Environmental Resource Compendium, \$10. PBS, Elementary and Secondary Service, 1320 Braddock Place, Alexandria, VA 22314. Telephone: (202) 955-5110.

Earth Child. A simple one-act play/musical designed to be a centre-piece for a two- to four-week miniunit on the environment. K-12 Peace Child International, 3977 Chain Bridge Road, Fairfax, Virginia 22030. Telephone: (703) 385-4494. 
Facets. Video distribution centre that carries hard-to-find environmental education videos. $1517 \mathrm{~W}$. Fullerton Avenue, Chicago, IL 60614. Telephone: 1-(800) 311-6197.

Film Distribution Centre rents environmental films and videos. 13500 NE 124 Street, Suite 2, Kirkland, WA 98034-8010. Telephone: (206) 820-2592.

The Lorax. Animated film about destruction of natural resources and pollution (also, see the Dr. Seuss book by this title-Random House 1971.) All ages. The Film and Video Library, University of Michigan, 400 Fourth St., Ann Arbor, MI 48103-4816, or Population Reference Bureau, Inc., 777 14th St., NW, Suite 800, Washington, DC 20005.

Media Network. Environmental education films and videos. Also distributes Greenjems, guide listing available environmental films and videos. $\$ 6.50$ individuals, $\$ 9.50$ institutions. 121 Fulton, 5th Floor, New York, NY 10038. Telephone: (212) 619-3455.

Worldlink: Spaceship Earth: Our Global Environment. First in a series of video travels around the world to present segments on environmental problems and solutions. Hosted entirely by young people. Includes teacher guide. 8755 W. Colgate Avenue, Los Angeles, CA 90048. Telephone: (213) 2732636.

\section{Electronic Bulletin Boards}

National Geographic Kids Network. Grades 4-6. Contact Dorothy Perreca, Project Manager, Kids Network, Educational Media Division, National Geographic Society, Washington, DC 20036.

Telephone: (202) 775-6580.

Eco-net is an electronic network for environmental information. Write: Institute for Global Communication, 3228 Sacramento St., San Francisco, CA 94115. Telephone: (415) 923-0900. 
-

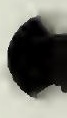


OH 541-2 A323 1991 GR-7-9

JUNIOR HIGH ENYIRONMENTAL AND

OUTOOOR EDUCATION TEACHER

RESOURCE MANUAL --

NL 40164250 CURR HIST

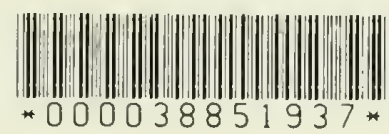

DATE DUE SLIP

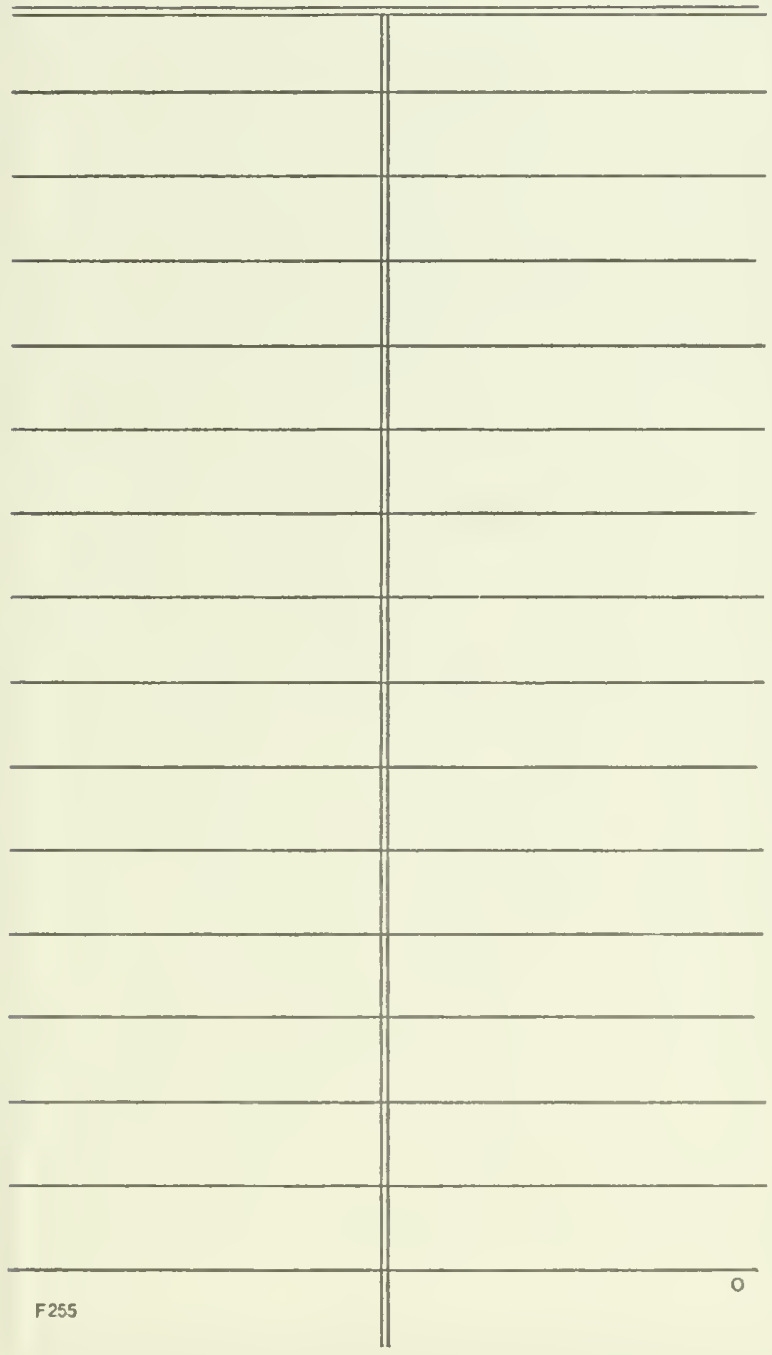


[1]

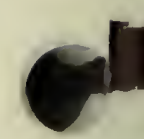

.

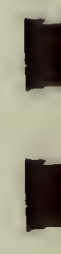

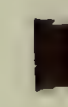

,

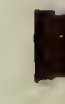

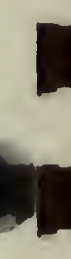

I

I

I

I

I

.

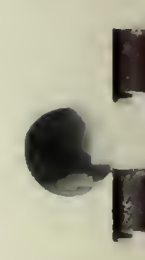

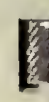

